\title{
Different Explanation, less frustration?
}

\author{
Citation for published version (APA):
}

Jie, J. L-J. (2020). Different Explanation, less frustration? making explicit whether implicit motor learning strategies are feasible and effective in neurological rehabilitation. [Doctoral Thesis, Maastricht University]. Ridderprint BV. https://doi.org/10.26481/dis.20200409jj

Document status and date:

Published: 01/01/2020

DOI:

10.26481/dis.20200409jj

Document Version:

Publisher's PDF, also known as Version of record

\section{Please check the document version of this publication:}

- A submitted manuscript is the version of the article upon submission and before peer-review. There can be important differences between the submitted version and the official published version of record.

People interested in the research are advised to contact the author for the final version of the publication, or visit the DOI to the publisher's website.

- The final author version and the galley proof are versions of the publication after peer review.

- The final published version features the final layout of the paper including the volume, issue and page numbers.

Link to publication

\footnotetext{
General rights rights.

- You may freely distribute the URL identifying the publication in the public portal. please follow below link for the End User Agreement:

www.umlib.nl/taverne-license

Take down policy

If you believe that this document breaches copyright please contact us at:

repository@maastrichtuniversity.nl

providing details and we will investigate your claim.
}

Copyright and moral rights for the publications made accessible in the public portal are retained by the authors and/or other copyright owners and it is a condition of accessing publications that users recognise and abide by the legal requirements associated with these

- Users may download and print one copy of any publication from the public portal for the purpose of private study or research.

- You may not further distribute the material or use it for any profit-making activity or commercial gain

If the publication is distributed under the terms of Article $25 \mathrm{fa}$ of the Dutch Copyright Act, indicated by the "Taverne" license above, 


\section{Different explanation, less frustration?}

Making explicit whether implicit motor learning strategies are feasible and effective in neurological rehabilitation 
The research presented in this thesis was conducted at:

The School for Public Health and Primary Care (CAPHRI), Department of Family Medicine, Maastricht University.

The School of Nutrition and Translational Research in Metabolism (NUTRIM), Department Nutrition and Movement Sciences, Maastricht University.

And

The Research Centre for Nutrition, Lifestyle and Exercise, Academy of Physiotherapy, Zuyd University of Applied Sciences

The research in this thesis was funded by Nationaal Regieorgaan Praktijkgericht Onderzoek SIA (RAAK-PRO, grant number 2014-01-49PRO) and Zuyd University of Applied Sciences.

The printing of this thesis was financially supported by the Scientific College Physical Therapy (WCF) of the Royal Dutch Society for Physical Therapy (KNGF).

Cover: $\quad$ Evenlien Jagtman

Lay-out: $\quad$ Li-Juan Jie

Printing: $\quad$ Ridderprint BV

ISBN: $\quad$ 978-94-6375-842-0

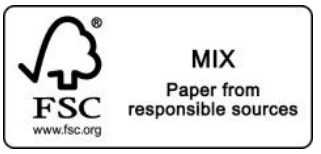

(C) Li-Juan Jie, Maastricht 2020

Chapters One, Four, Five, Six, and Eight are licensed under the Creative Commons Attribution 4.0 International License (http://creativecommons.org/licenses/by/4.0/) which permits unrestricted use, distribution, and reproduction in any medium, provided the original work is properly cited. The licenses of the chapters Two, Three and Seven of this $\mathrm{PhD}$ thesis are indicated on the individual chapter pages.

Note: Some chapters have been published in British and others in American journals, the used language form is therefore not uniform. Some words may have been spelled in UK and other in US-English. 


\section{Different explanation, less frustration?}

Making explicit whether implicit motor learning strategies are feasible and effective in neurological rehabilitation

\section{PROEFSCHRIFT}

ter verkrijging van de graad van doctor aan de Universiteit Maastricht, op gezag van de Rector Magnificus, Prof dr. Rianne M. Letschert volgens het besluit van het College van Decanen,

in het openbaar te verdedigen

op vrijdag 3 juli 2020 om 10.00 uur

door

Jolande Li-Juan Jie

Geboren op 13 juli 1991

te Lanaken, België 


\section{Promotor}

Prof. dr. A.J.H.M. Beurskens

\section{Copromotoren}

Dr. S.M. Braun (Zuyd Hogeschool)

Dr. K. Meijer

\section{Beoordelingscommissie}

Prof. dr. R.A. de Bie (voorzitter)

Dr. S. Aarts

Prof dr. A.F. Lenssen

Dr. E.C. Kal (Brunel University, Londen)

Prof dr. B. Steenbergen (Radboud Universiteit Nijmegen) 


\section{TABLE OF CONTENTS}

Chapter 1 General Introduction 1

Chapter 2 Analogy learning in Parkinson's disease: A proof-of-concept 9 study

International Journal of Therapy and Rehabilitation, 2016; 23(3), 123-130.

Chapter 3 The immediate influence of implicit motor learning strategies on spatiotemporal gait parameters in stroke patients: a randomized within-subjects design Clinical rehabilitation, 2019; 33(4), 619-630.

Chapter 4 The Effects of Implicit and Explicit Motor Learning in Gait Rehabilitation of People After Stroke: Protocol for a Randomized Controlled Trial JMIR research protocols 2018; 7 (5), e142

Chapter 5 Implicit and explicit motor learning interventions have similar effects on walking speed in people after stroke: a randomized controlled single blind

Trial

Manuscript submitted

Chapter 6 Implicit and explicit motor learning interventions for gait in people after stroke: a process evaluation on fidelity and feasibility performed alongside a randomized controlled trial. Manuscript submitted

Chapter 7 Design of the user interface for "Stappy", a sensor-feedback system to facilitate walking in people after stroke: a usercentred approach

Disability and Rehabilitation: Assistive Technology, 2019, 1-9.

Chapter 8 General Discussion

English Summary

Nederlandse Samenvatting

Valorisation

Acknowledgements / Dankwoord

Curriculum Vitae

About the Author 

CHAPTER 1

General Introduction 
Long before we could speak, explain or describe what we were doing, we were able to respond and adapt to the environment more or less 'automatically'. For example, when being threatened by a predator, we would likely have responded with either a fight or flight response.1 For our ancestors, performance failure would have had catastrophic consequences. Therefore in order to survive, evolution selected advantages of implicit, unconscious learning.2,3 Today, we still face dangerous or stressful events e.g. in (car) accidents or when taking the deciding penalty in the final of the world championship. The advantages of implicit motor learning, such as robust performance in pressurised or multitask environments can help us to cope with these specific circumstances.

Implicit motor learning is seen as a learning approach that takes place in a less conscious manner and can be defined as "learning which progresses with no or minimal increase in verbal knowledge of movement performance (e.g., facts and rules) and without awareness".4 It is also often explained as the anthesis of explicit motor learning or other end of the implicit-explicit continuum. Explicit motor learning is characterised by a learning process that is very conscious by nature; learners are usually able to precisely describe the underlying facts and rules that were used to perform the motor skill. For example, learning a tennis service through explicit motor learning would mean that the coach would provide you with many facts and rules e.g. explaining how to hold your racket, how to throw the ball, how to position your body, where to hit the ball, etc. This more explicit approach closely relates to more traditional models of motor learning e.g. as proposed by Fitts and Posner.5 They describe three stages of motor learning. The first 'cognitive' stage is characterised by the learner trying to understand the motor skill e.g. through detailed instructions and feedback by a coach. After practice the motor skill becomes gradually more stable and consistent (associative stage) until often after years of practice the motor skill becomes fully automatic and can be performed without conscious thoughts (autonomous stage).

\section{Motor learning in neurological rehabilitation}

Motor learning also plays an essential role in neurological rehabilitation. For instance, people with a non-acquired brain injury (e.g. after Stroke) or people with chronic neurological disorders (e.g. Parkinson's disease) frequently need to (re)learn daily life motor skills, such as walking, taking the stairs or standing up from a chair. These activities are important to patients as they increase autonomy and are often criteria for discharge to the home environment. Health care professionals e.g. physiotherapists use motor learning strategies to structure their training sessions. Currently these therapy sessions seem to be very explicit in nature. Johnson and colleagues 6 observed that therapists who provided gait training to people after stroke used large amounts of verbal instructions and feedback (on average every 14 seconds). Frequently these instructions were directed at thinking about 'how' they are moving.6 Receiving many verbal instructions about how a person should perform a motor skill, closely relates to explicit motor learning. Explicit learning requires 
explicit, declarative knowledge, and is therefore assumed to be highly dependent on cognitive, working memory, resources.

\section{Cognitive demands and potential benefits of implicit motor learning}

A commonly described cognitive resource during (explicit) motor learning is working memory. Working memory is responsible for holding and processing information and has a limited capacity.7 Given that these resources are limited, it can be difficult to perform several tasks simultaneously, especially if cognitive demands of one of these tasks is high. Implicit motor learning strategies have been hypothesised to circumvent information processing of declarative, explicit knowledge during the early phases of learning. Due to minimal reliance on working memory resources, it may be easier for implicit learners to cope with multi-task situations.

Numerous studies, of which many have been applied within sports settings, have examined the benefits of implicit motor learning (e.g. for a review see). Implicit motor learning has been found to be less reliant on individual differences such as age and IQ.9 Research has also shown that implicitly acquired motor skills in healthy subjects are more stable in multi-task situations10-12, under conditions of aerobic fatigue 3 and in pressurised or anxious environments11-14. These potential benefits make implicit motor learning so inviting to use within daily life practice. A possible disadvantage may be that for most implicit paradigms, the rate of learning seems lower in the early phases of motor learning compared to explicit motor learning.2,15,16

\section{The application of implicit motor learning}

Recent literature suggests that implicit and explicit learning can been as the extremes on a continuum rather than a distinct dichotomy.4 Depending on how motor learning strategies are operationalised, learning approaches may be seen as more implicit or more explicit. In the following paragraph, the application of different implicit learning paradigms are described.

Within the field of cognitive psychology, various experiments have demonstrated that learning can take place implicitly. Within these experiments, people improve performance because they have unconsciously learned to recognize certain patterns or structures such as ordered sequences of letters as generated by artificial grammars.17 Most people are unaware of the repeating sequence in the task i.e. they cannot recall the sequence, which suggests that the sequence has been learned implicitly. Although they nicely illustrate the concept of implicit motor learning, these non-functional computer tasks 17,18 do not reflect real life activities. In recent years, due to the potential benefits, implicit motor learning has been explored within a wide range of settings e.g. within sport contexts11,12, child development 19 , surgery20,21, speech therapy22 and rehabilitation settings23-25. Masters was the first to develop an implicit paradigm for a sports related motor task, namely golf- 
putting.12 A learning condition was designed to prevent learners from 'thinking' about or 'testing hypothesis' regarding their movement performance. This process has been described as 'reinvestment' and places high demands on working memory resources. Participants were asked to perform a golf putting task while simultaneously completing a random letter generation task. By performing the secondary task, the opportunity to accrue verbal knowledge or 'reinvest' in their movement performance was minimised. It was therefore assumed to by-pass the high demands on working memory. In the years that followed, next to dual-task learning, also other implicit motor learning paradigms were developed, such as errorless learning, analogy learning or observational learning. Errorless learning can be explained as learning while avoiding or reducing errors in performance. For example, Poolton et al26 used an errorless condition in (again) a golf putting task. Mistakes were minimised by starting very close $(1 \mathrm{~m})$ to the putting hole, then slowly the distance was increased $(1.5 \mathrm{~m}, 2.0 \mathrm{~m}, 2.5 \mathrm{~m}$ and finally $3.0 \mathrm{~m})$. Successful performance, as in the absence of errors, may be less dependent on hypothesis testing leading to implicit motor learning. Analogy learning, is another paradigm that has shown characteristics of implicit motor learning. In analogy learning, the learning process is facilitated by metaphors or analogies.11 For example, Lam (and colleagues), used the analogy instruction "Shoot the ball as if you are trying to put cookies into a cookie jar on a high shelf" to guide learners in how to impart backspin during a basketball shooting task.10 Although an analogy is presented as a verbal instruction, it is assumed to rely very little on processing verbal (explicit, declarative) knowledge and may therefore be seen as a more implicit approach. Observational learning can be described as learning by observing a movement. Through observation, the learner determines the key spatiotemporal characteristics of the task.27 This is a strategy that children often use naturally; they observe how a motor skill is being performed and then they try to copy that behaviour. Also, adults can improve performance implicitly through observation of a demonstration. For example, people have learned how to perform a surgery (suturing and knot-tying) task by observation alone. Verbal instructions are thus not necessarily needed to learn a motor task.20

\section{The potential benefits of implicit motor learning for neurological rehabilitation}

Physiotherapists face the challenge on a daily basis to deliver effective therapy in the most efficient manner. Motor learning is an important part of rehabilitation. Preferably, the (newly) learned motor skills are also durable over time and resilient against the potential disturbing effects of anxiety or in multi-task situations. In contrary to explicit motor learning, motor skills that were learned implicitly have shown to be robust during these specific circumstances. So far, the benefits of implicit motor learning have mainly been explored within healthy young adults in sports related motor tasks. The question therefore remains, why is it interesting to explore the implicit motor learning within the neurological population? 
It is important to realise that next to physical impairments patients within neurological rehabilitation also often experience difficulties with a variety of cognitive functions. For example, people after stroke often have to cope with memory, attention or concentration deficits.28 The title of this thesis 'different explanation, less frustration' refers to the idea that receiving and processing many detailed, explicit instructions can lead to frustration, especially when dealing with cognitive impairments. A different, less cognitive demanding explanation i.e. implicit motor learning, may perhaps be an easier learning approach for people with cognitive impairments compared to more traditional (explicit) motor learning approaches. Although from a theoretical perspective, the application of implicit motor learning within neurological population seems promising, at the start of this project (four years ago), little was known about the translation of these effects towards clinical practice. Some studies explored the capability to learn implicitly (e.g.23,29) and potential benefits of implicit learning (e.g.25) in people after stroke. However, there is a lack of Randomised Controlled Trials (RCTs) that examine the effectiveness and feasibility of implicit motor learning in clinically relevant tasks. An important preliminary step, before conducting a larger trial within clinical practice, is to establish the potential effects and feasibility within a laboratory setting.30 This knowledge can then be used to set up and develop a trial to examine the effectiveness of implicit motor learning within the neurological population and daily life environment.

\section{Practice at home}

Not only the right treatment approach, but high-intensity, repetitive and task-specific training are important requirements for effective therapy and sustainable results within stroke rehabilitation.31 Also, after discharge from hospitals or inpatient rehabilitation facilities it is important to keep therapy intensive. To optimise training intensity, therapists often encourage their patients to also practice their motor skills alone at home, outside the guided therapy sessions. Yet, the exercise compliance is often low; some people forget to practice, or the lack of motivation or feedback can be reasons to not practice unsupervised.32 Once people return to their home environments, their training intensity often significantly reduces. Innovative, simple technologies may be used to support patients during independent practice or exercises at home.33 Technologies, such as sensorfeedback systems,34 can be a support outside the guided therapy sessions by providing patients with real-time and personalised feedback during practice. However, research shows that many technologies are not always used as much as intended, as there are many factors that can influence the actual use of technologies.35 For example, a boring or complicated technology may lead to a lack of motivation to use the technology. Whereas creating a meaningful and enjoyable user-interface for the target population may be a solution to optimize the user experience and thereby the actual use of the technology. Rather than focusing on developing new technologies, it may sometimes be more efficient to optimize promising existing systems e.g. through (re)designing meaningful userinterfaces. 


\section{Aim of the thesis}

The overall aim of this $\mathrm{PhD}$ thesis is to develop and explore the application of different implicit motor learning approaches, and to assess the effectiveness and feasibility of one promising implicit motor learning approach at home for people who suffered from a stroke. Additionally, the design of a user interface for a simple innovative technology that can be used to support independent practice of walking at home, independent of the approach, is developed.

To answer the overall aim, the thesis is divided into three phases. Phase 1 focuses on exploring the application of different implicit motor learning approaches and delivers input for the second phase. In phase 2 the protocol, effectiveness and process evaluation of a Randomised Controlled Trial (RCT) is described. In the RCT, an implicit motor learning approach is compared to an explicit motor learning approach. Finally, in the last phase, the (re)design process of a user-interface for an existing sensor-feedback system that supports people after stroke during walking is described.

\section{Outline of the thesis}

The outline of this thesis is presented in Figure 1. Within this thesis, all studies focused on the functional task 'walking'. The first studies (chapters 2 and 3) were performed within a laboratory setting, whereas the following studies (chapters 4 to 7) focused on the home environment of the participants. In chapter 2 the feasibility and potential benefits of an analogy learning intervention to facilitate stride length regulation are described. In chapter 3 the immediate changes in walking performance associated with three implicit motor learning strategies and patient experiences within each strategy are explored. These chapters provided insight and knowledge about the application of implicit motor learning within this context and formed the basis for setting up a randomised controlled trial. Chapter 4 describes the protocol of this randomised controlled trial, including a detailed description of the implicit and explicit motor learning walking interventions. In chapter 5 the results of the trial are described and in chapter $\mathbf{6}$ the interventions, therapists' and patients' experiences of the trial are evaluated. Additionally, this thesis describes an easyto-use sensor-feedback system which supports patients when practicing walking alone in their home environments. In Chapter 7 the design process of the user-interface for an easy-to-use sensor-feedback system is described. The overall findings of this thesis are discussed in Chapter 8.

6 | General Introduction 




Figure 1. Outline of the thesis divided over three phases. 



\section{CHAPTER 2}

\section{Analogy learning in Parkinson's disease: A proof-of- concept study}

Li-Juan Jie, Victoria Goodwin, Melanie Kleynen, Susy M Braun, Michael

Nunns, Mark Wilson

Published as:

Jie LJ, Goodwin V, Kleynen M, Braun S, Nunns M, Wilson M. Analogy learning in Parkinson's disease: A proof-of-concept study. Int J Ther Rehabil. 2016;23(3):123-130.

Open access version of the accepted manuscript is available at HBO-kennisbank doi: 10.12968/ijtr.2016.23.3.123

() 2016, MA Healthcare Ltd. All rights reserved. 


\begin{abstract}
Background/Aims: Analogy learning is a motor learning strategy that uses biomechanical metaphors to chunk together explicit rules of a to-be-learned motor skill. This proof-ofconcept study establishes the feasibility and potential benefits of analogy learning in enhancing stride length regulation in people with Parkinson's disease.
\end{abstract}

Methods: Walking performance of thirteen individuals with Parkinson's disease was analysed using a Codamotion analysis system. An analogy instruction: 'following footprints in the sand' was practised over eight walking trials. Single- and dual-task (motor and cognitive) conditions were measured before training, immediately after training and 4 weeks post training. Finally, an evaluation form was completed to examine the intervention's feasibility.

Findings: Data from 12 individuals ( 6 females and 6 males, mean age 70, Hoehn and Yahr grade I-III) were analysed; one person withdrew due to back problems. In the single-task condition, statistically and clinically relevant improvements were obtained. A positive trend towards reducing dual-task costs after the intervention was demonstrated, supporting the relatively implicit nature of the analogy. Participants reported that the analogy was simple to use and became easier over time.

Conclusions: Analogy learning is a feasible and potentially implicit (i.e. reduced working memory demands) intervention to facilitate walking performance in people with Parkinson's disease.

10 | Analogy learning in Parkinson's disease 


\section{INTRODUCTION}

Parkinson's disease neurodegenerative disorder primarily due to dysfunction of the basal ganglia, which manifests mainly as four cardinal symptoms: tremor, rigidity, postural instability and bradykinesia.36 Despite optimal medication therapy, many people with Parkinson's disease still experience gait impairments.37 A Parkinson's gait is typically characterised by a reduced velocity and reduced stride length, while cadence (steps per minute) control may be relatively unaffected 38 Reduced attentional capacity is another common feature in people with Parkinson's disease 39 ; affecting their ability to perform dual-tasks required for effective daily life (e.g. walking and talking simultaneously).40 Reduced attentional capacity is also related to an increased risk of falls.41 The consequences of falls, such as reduced confidence, mobility and quality of life 42 greatly affect people's daily life. Therefore, there is a need to develop interventions to improve walking performance in people with Parkinson's disease.

Cognitive strategies, such as attentional strategies (self-generated with an internal focus), the use of external cues (auditory, visual or tactile), or self-instruction strategies can be beneficial in improving gait.43 In Parkinson's disease, skills acquired via cognitive strategies will mostly not become automated, but will remain under conscious control and may be guided via the use of cues.44 From a neurophysiological perspective it is proposed that such consciously acquired, explicit learning strategies may reroute a non-automatic pathway that compensates for the damaged basal ganglia (responsible for internal generation of movements).

While cognitive strategies are frequently recommended to optimise motor learning in people with Parkinson's disease, 45 the attentionally demanding nature of such techniques may impair multitasking performance. As cognitive strategies require working memory capacity, their effectiveness may be reduced when working memory processing is already required to perform secondary tasks, due to attentional overload (i.e. when attentional demands exceed working memory capacity).46 Indeed, studies using cognitive strategies to support performance in dual-task situations reveal equivocal results, 47 possibly due to variations in the type (e.g. motor or cognitive) and complexity (cognitive demand) of the dual tasks used. As rehabilitation involves training within a functional context in which multi-task situations frequently occur, it is important that alternative approaches to motor learning are explored.

One such approach that has received recent interest in a variety of movement domains is implicit motor learning.8,12 This approach evokes evolutionary heuristics that reflect that much of the way in which humans respond and adapt to the environment occurs implicitly (i.e. without conscious awareness and often without intention). It is unsurprising that 
evolution has selected advantages of implicit (unconscious) learning, given that learning is a biological imperative that provided our ancestors a significant survival advantage.48,49

Research has shown that implicit learning is less dependent on individual differences such as age or IQ, is more robust under stress or dual-tasking conditions, and, is more durable over time. 9 Implicit motor learning is therefore a potentially useful strategy for people with Parkinson's disease, as it strives to minimise the cognitive load in working memory when performing a motor task.50 Different strategies to induce implicit motor learning exist, including dual-tasking, errorless learning, manipulating feedback, and analogy learning; however, research validating these strategies has mainly been carried out with healthy, unimpaired populations. 8

Analogy learning involves repackaging relevant bits of (explicit) information of the to-belearned skill into one integrated biomechanical analogy or metaphor.11,51 Although an analogy is provided as a verbal instruction, it is not presented as an explicit set of rules to guide the execution of the desired movement pattern. Instead, analogies strive to combine explicit rules into meaningful chunks of information, which utilise fast, unconscious (working memory independent) processing.11,52 For example, the analogy of 'reaching your hand into a cookie jar' describes the appropriate wrist snap required to impart backspin on a basketball during the performance of a free-throw, without describing the step by step rules to create the backspin.10

For people with Parkinson's disease, there may be two potential advantages of analogy learning. First, analogies have practical benefits that make them extremely flexible to use in different environments. In comparison, visual cues (e.g. stripes marked on the floor) have to be set out in advance on regularly taken pathways (usually only in the home), and auditory cues require delivery via an earphone, making communication difficult. In analogy learning, however, once an appropriate analogy has been found, this can be used anytime and independent of additional material support. Second, the implicit nature of analogy learning may improve the ability of individuals to multitask and perform under more demanding conditions.11 Research has demonstrated that implicit motor learning techniques free up cognitive resources from step-by-step movement control, and these can be redeployed to other tasks.10,11

From both a theoretical and practical perspective, analogy learning seems a promising intervention, but its application and feasibility in Parkinson's disease rehabilitation has yet to be examined. Therefore, the following research questions for this proof-of-concept study were established: Can analogy learning facilitate walking performance in people with Parkinson's disease? Is this a feasible intervention in therapeutic practice? We hypothesise that participants will have increased walking velocity and longer strides 
following exposure to analogy learning, and that attentional costs of dual-tasking will be reduced after training.

\section{METHODS}

\section{Participants}

Ethical approval was obtained from the University of Exeter (Sport and Health Science department). The study was promoted in collaboration with Parkinson's UK. Interested individuals contacted the first author via phone or e-mail. Participants were included if they self-reported a shuffling gait and were able to walk independently without walking aids for at least 15 minutes (with resting breaks). Participants were excluded if they reported other medical problems affecting gait, or were receiving any treatment/rehabilitation to improve walking performance. During the study period participants were asked not to take part in additional physiotherapy programmes. Participants had up to five working days to consider participation, and written informed consent was obtained. The study took place in the gait laboratory at the University of Exeter, requiring two visits, spaced 4 weeks apart.

\section{Procedures and data collection}

Pilot testing with the first participant was performed to fine-tune an appropriate analogy,24 and to determine the duration of the training blocks in the walking protocol. The 'footsteps in sand' analogy was clear and understood immediately by the participant; therefore, it was deemed to be appropriate for the study. It was observed by the researchers and confirmed with the participant that the original protocol (four blocks of training) was too long, resulting in tiredness. Therefore, the protocol was shortened from four to two training blocks. The initial participant was included in the study, deleting the last two training blocks, as this did not influence the data analysis, or our interpretation of the results.

Demographic data (age, gender, Hoehn and Yahr stage, medication use) were collected during the initial visit. Participants were set up with Codamotion (Charnwood Dynamics, Leicestershire, UK) active markers attached non-collinearly on both feet at the heel bone and first and fifth metatarsals. Then a single standing trial (5 seconds) and multiple walking trials were performed over different conditions (see Table 1). 
Table 1. Overview of walking trials in sessions 1 and 2

\begin{tabular}{ll}
\hline Conditions session 1 & Conditions session 2 \\
\hline Single task & Single task \\
Motor dual task & Motor dual task \\
Cognitive dual task & Cognitive dual task \\
Training Block 1 & \\
Training Block 2 & \\
Motor dual task & \\
Cognitive dual task & \\
\hline
\end{tabular}

First, a single task (only walking) in the form of a 10-metre walking test was performed. This was followed by a motor dual task, in which participants were asked to walk while carrying a tray with empty plastic cups53; and a cognitive dual task, that required subtracting in threes (out loud) from a random (to prevent familiarisation) three-digit number.54 Following these baseline measures, participants were exposed to the analogy instruction (see following section) and practiced this analogy over two training blocks (eight walking trials). Finally both dual task conditions were repeated.

Every condition contained four walking trials in which participants were asked to walk at a comfortable walking speed. Walking took place over a $10 \mathrm{~m}$ distance, of which the middle $5 \mathrm{~m}$ was recorded by the two Codamotion CX1 units at $200 \mathrm{~Hz}$, which demonstrates good inter-and intrarater and intersession reliability for 3D kinematics.55 A video camera (Panasonic SDR-S70) continuously recorded walking performance over the entire session, and velocity was manually calculated by timing the walking distance (10 m) using a stopwatch. Stride length was computed using a custom Matlab script (version 2012a, The Mathworks, US). Mean velocity (ms-1) and stride length (m) in the 10-metre walking test (single task) and dual tasks were used to assess walking performance.

At the start of visit two, participants completed an evaluation form and then went through the same set-up procedure as in visit one. The evaluation form assessed their subjective experiences of the analogy intervention; involving questions targeting adherence, applicability, and perceived improvements (Table 2). Responses were recorded using binary outcome variables 'yes/no', scales ranging from $0-10$, and free comments. Session two did not involve any training but only the 10-metre walking test and dual-task trials. Visit one lasted for approximately 60 minutes and visit two lasted for approximately 30 minutes. 


\section{The analogy instruction}

The analogy instruction was presented to participants pictorially (Figure 1) in association with the following instruction: 'Do you see the footprints in the sand? Now while walking, pretend that you are following footprints in the sand as you walk'

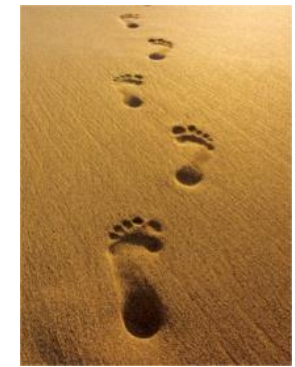

Figure 1. Picture of footprints in the sand.

Participants were instructed to practice at home by incorporating the analogy into their everyday walking, although no specific training exercises were prescribed.

\section{Data analysis}

Demographic data were reported descriptively (mean, standard deviation). Individual walking performance was presented by plotting individual delta (change) scores for each time point compared to baseline. Repeated analysis of variance measures, comparing each time point (baseline, training blocks 1 and 2, and at 4 weeks) for the single task, was performed to explore group improvements in velocity and stride length. If the assumption of sphericity was violated, a Greenhouse-Geisser correction was applied. Estimated effect sizes $\left(\eta_{\mathrm{p} 2}\right)$ were calculated using partial eta squared and (least significant difference) post hoc tests were used to explore effects.

The effects of secondary tasks on walking performance were explored by calculating the dual task cost at each time point (pre, post and 4 weeks). The dual task costs for velocity and stride length are expressed as a percentage of single task performance using the following formula47,56:

Dual task cost $=($ dual task - single task(baseline $)) / \operatorname{single~task(baseline)~} * 100$

Reliable differences from zero at each time point were assessed for dual task cost using one-sample t tests.56

As this was a proof-of-concept study, it was not formally powered for inferential comparisons. Exploratory analyses were undertaken to estimate the mean between group differences (and 95\% CI) for outcomes. Velocity increases of $0.05 \mathrm{~ms}-1$ and stride length increases of $0.048 \mathrm{~m}$ were set as clinically relevant improvements (see discussion).57,58 
Participant's evaluations were reported descriptively. Free comments were used to describe personal experiences of the participants and clarifying examples were quoted.

\section{RESULTS}

In total, 13 people with Parkinson's disease (Hoehn and Yahr scale I-III) were included in the study (7 females, 6 males) with a mean age (SD) of $70( \pm 7)$ years. One participant withdrew during the study due to back problems, leaving 12 participants to be included in the subsequent analysis.

\section{Effect of analogy on single task walking}

Figure 2 demonstrates walking performance of each individual. Most participants showed an initial drop in velocity, with a simultaneous increase in stride length. At 4 weeks, most participants achieved improvements for both velocity and stride length.
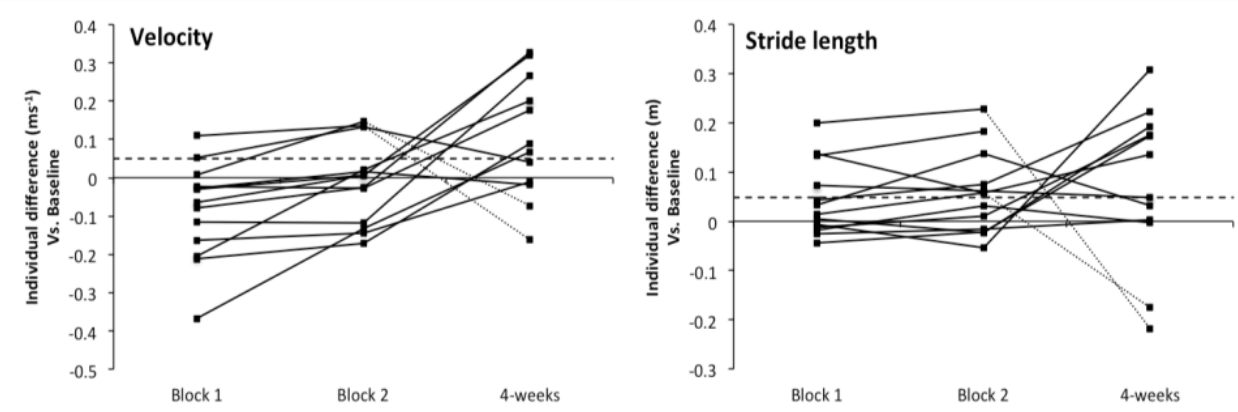

Figure 2. Individual walking performance in the single task condition for velocity (left) and stride length (right). Data is presented as change (delta) scores of each time point (block 1, block 2 and 4week retention) compared to baseline. Horizontal dashed lines indicate the meaningful threshold for clinically relevant improvements.

As a group, for velocity, statistically significant results between the conditions were obtained $\left(\mathrm{F}_{(1.47,16.18)}=5.32, P=0.02, \eta_{\mathrm{p} 2}=0.33\right)$. LSD pairwise comparisons demonstrated that compared to baseline $(0.89 \pm 0.14 \mathrm{~ms}-1)$, velocity significantly decreased at training block $1(0.80 \pm 0.16 \mathrm{~ms}-1, P=0.04)$, and increased at 4 weeks $(0.99 \pm 0.15 \mathrm{~ms}-1, P=0.046)$. Velocity at 4 weeks was also significantly greater than at block $1(P=0.01)$. For stride length no significant main effect was obtained $\left(\mathrm{F}_{(1.17,12.86)}=1.08, P>0.5, \eta_{\mathrm{p} 2}=0.09\right)$. Group performance in the single task conditions, including confidence intervals are demonstrated in Figure 3.

Two participants (6 and 11) reported experiencing a 'bad day' during visit two, resulting in increased festination and impaired walking (dotted lines, Figure 2). Post hoc sensitivity 
analysis, excluding these two participants resulted in highly significant outcomes and large effect sizes in the predicted direction for both velocity $\left(\mathrm{F}_{(3,27)}=14.73, P<0.001, \eta \mathrm{p}=\right.$ $0.62)$ and stride length $\left(\mathrm{F}_{(1.41,12,68)}=7.51, P=0.01, \eta p=0.46\right)$.
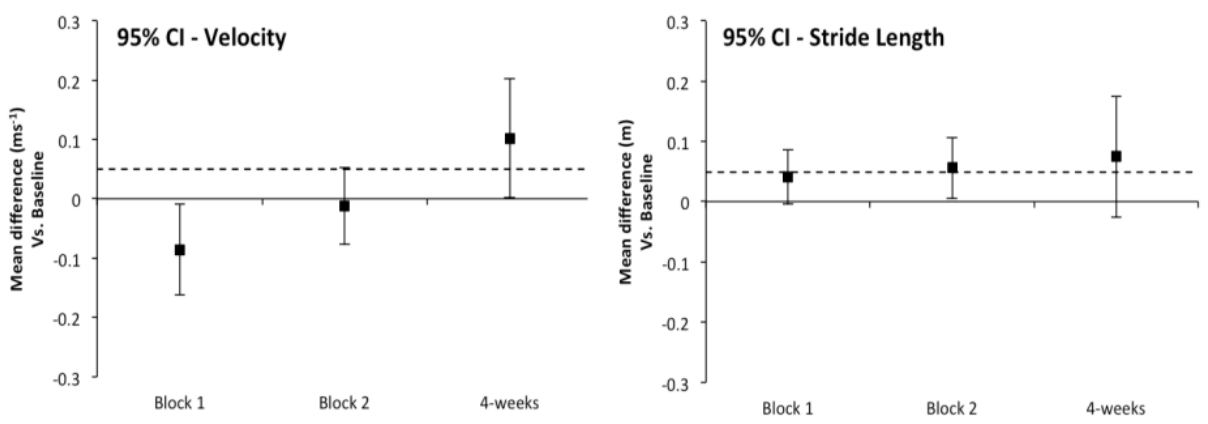

Figure 3. Mean ( $\pm 95 \%$ C.I.) group performance in the single task condition for velocity (left) and stride length (right). Data is presented as change (delta) scores of each time point compared to baseline. Dashed lines indicate the meaningful threshold for clinically relevant improvements.

\section{Effect of analogy on dual tasking while walking}

The proportional dual-task costs are presented in Figure 4. At baseline, the dual-task costs for velocity were reliably different from zero in the motor- $(\mathrm{t}(12)=-2.43, P<0.05)$ and cognitive $(\mathrm{t}(12)=-2.43, P<0.05)$ dual-task conditions. For stride length, only the cognitive dual-task cost $(\mathrm{t}(12)=-2.72, P<0.05)$ at baseline was reliably different from zero.

Post training, the mean costs were not reliably different from zero for either performance measure. At 4 weeks post training, dual-task performance was actually better than baseline single task performance (though not significantly so).
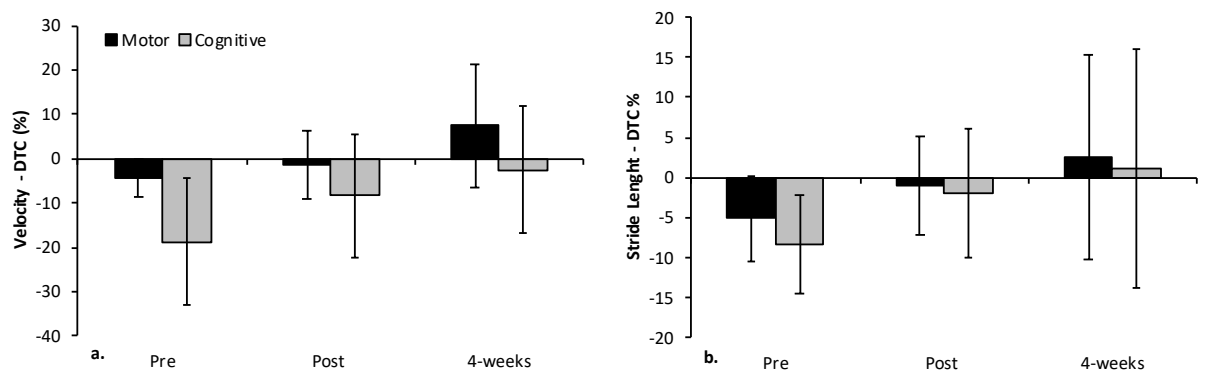

Figure 4. Mean ( $\pm 95 \%$ C.I.) dual task cost (DTC) for velocity (a) and stride length (b) for the motor and cognitive secondary task conditions. 


\section{Participant evaluation}

Participant responses are reported in Table 2. People reported having to think a lot while using the analogy, but also reflected that it became easier over time 'At first yes and then it becomes second nature' (Participant 5). However others reported, 'It's so simple that it doesn't matter' (Participant 11). One participant reported that the analogy intervention did not bring any improvements and commented with 'I don't feel any difference' (Participant 3 ), whereas others reported gaining focus, control and stability, as well as feelings of confidence.

Some participants pointed out that they would only use the analogy incidentally, only when situations when walking deteriorated, whereas others stated using the analogy daily. Only four participants had used cues previously and with varying degrees of success. Participant 4 had used marching as a strategy, 'but this was very mechanical. I prefer walking while following the footprints because it is more natural'.

Table 2. Responses $(n=12)$ from the evaluation form, at 4-weeks.

\begin{tabular}{|c|c|c|}
\hline \multirow[t]{2}{*}{ Question } & \multicolumn{2}{|l|}{ Response } \\
\hline & $\begin{array}{l}\text { Mean } \pm \mathrm{SD} \\
\text { (range) }\end{array}$ & $\begin{array}{l}\text { Agreed } \\
(n)\end{array}$ \\
\hline How often did you practice the analogy at home? (days/week) & $6 \pm 1(3)$ & - \\
\hline Is it difficult to use the analogy? ( 0 not -10 very difficult) & $3 \pm 2(4)$ & - \\
\hline $\begin{array}{l}\text { Does it become easier to use the analogy the more you } \\
\text { practice / use the analogy? }\end{array}$ & - & 9 \\
\hline $\begin{array}{l}\text { Do you need to think a lot when using the analogy? }(0 \text { not - } \\
10 \text { very hard) }\end{array}$ & $4 \pm 2(7)$ & - \\
\hline Is the analogy difficult to visualise? ( 0 not -10 very difficult $)$ & $3 \pm 2(7)$ & - \\
\hline Do you enjoy going to the beach / walking through sand? & - & 10 \\
\hline Did the analogy bring any improvements? & - & 11 \\
\hline Will you use the analogy in the future? & - & 12 \\
\hline $\begin{array}{l}\text { Have you used different analogies before or are you using } \\
\text { other analogies at the moment? }\end{array}$ & - & 4 \\
\hline
\end{tabular}

18 | Analogy learning in Parkinson's disease 


\section{DISCUSSION}

This is the first proof-of-concept study exploring analogy learning in people with Parkinson's that investigated the feasibility and potential benefits of this intervention on walking performance.

\section{Walking performance: single task}

For people with Parkinson's, clinically important differences in walking velocity range from 0.05-0.22 ms-1.58 For older people, meaningful changes in step length are estimated in a range from 0.24-0.61 ms-1.57 Strides were used rather than steps, therefore we proposed a conservative threshold of $0.48 \mathrm{~m}$ for stride length and $0.05 \mathrm{~ms}-1$ for velocity. In line with the hypothesis that analogy learning would increase walking velocity and stride length, statistically and clinically (i.e. exceeding the meaningful threshold), significant improvements for velocity were obtained. The findings for stride length failed to reach statistical significance, but they revealed clinically relevant improvements $(n=7)$ and demonstrated positive trends in the predicted direction (i.e. increased stride length following intervention; Figure 2).

In interpreting these results, it is important to recognise that the current study was designed to establish 'proof of concept' that analogies have potential clinical benefits for this population and was not powered to confirm statistical significance. Additionally, it is important to note that we included the data of two participants who reported having 'a bad day' at the 4-week follow-up in the statistical analysis, and it was evident from the sensitivity analysis that they negatively influenced the overall result. Despite these concerns, the magnitude of performance improvements found in this brief intervention is similar to those obtained in studies using cues to facilitate walking. 59

The current study observed most performance improvement in velocity during visit two (4 weeks later) rather than directly at visit one. This is in contrast with other analogy studies, which have tended to find immediate improvements after training.11,51 While it is evident that participants were able to make some initial improvements in stride length, we suggest that participants require time to fully assimilate the analogy and slow down initially in order to focus on their stride lengthening. Once stride regulation has been internalised, they are able to increase velocity and maintain their new stride length (Figures 2 and 3).

An alternative explanation may be related to differences in training dose, with some previous analogy learning using up to 3000 repetitions.11 However, as we worked with patients rather than young healthy individuals, similar amounts of repetitions within one session were not feasible for safety reasons (as determined via the initial pilot testing). One clear difference between analogy learning for sport (where most of the research has 
been applied) and for therapy is that safety is the critical factor in therapy, whereas the rate of skill acquisition is prioritised in sport. The nature of the different study populations may also contribute to the different findings. Whereas the existing literature sampled from young, healthy people, our study comprised older people with a neurodegenerative disorder who are known to take longer to learn motor skills.60

\section{Walking performance: dual tasks}

At baseline, interference effects were obtained for both dual-task conditions, indicating that the task difficulty was appropriate (Figure 4). However, at immediate and 4-week post test conditions, there was no significant cost of dual- tasking, suggesting that participants were now able to free up resources from the walking task to complete the secondary tasks. The reduction in the dual-task cost of walking in the motor dual task (carrying glasses on a tray), importantly demonstrate the functional transferability of analogy learning to a daily life task.

Additionally, the dual-task cost data for the cognitive dual task condition reflect a positive trend towards the analogy exhibiting the implicit characteristic of robust performance under secondary task loading.9 However, the current study cannot claim that the improvements in walking performance are due solely to the intervention, as performance could also have improved due to the multiple exposures to the same testing conditions. The dual tasks demonstrated a relatively large 95\% confidence interval at 4 weeks (Figure 4), which may be explained by the large variability in performance within the group (two influential data points) and the small sample size of the study. Future research should include a control group (receiving an explicit, cognitive intervention) to determine if analogies provide relative benefits in multitasking performance over other techniques.

\section{Feasibility of the analogy intervention}

Generally, participants were positive about the analogy intervention, finding it relatively easy to use and perceiving noticeable improvements in their gait. Most participants perceived the walking on the beach analogy positively. Although one participant reported experiencing no improvements in walking (Table 2), walking performance at 4 weeks revealed positive changes in the objective measures of interest. This discrepancy between objective measurement, self-report and subjective perceptions highlights the need for mixed- methods evaluations using a range of data collection methods to fully understand the impact of interventions from the perspective of the patient and the clinician. While all 12 participants reported that they would continue to use the analogy, there were differences in terms of whether they responded with 'daily/ frequently' or on a more 'situation dependent' basis that appeared to be related to the degree of gait impairment they experienced in the baseline test. Participants with lower velocity were likely to use the analogy more frequently, whereas those with better initial performance stated that they would use the analogy when their walking deteriorated. This finding reveals an important 
additional benefit of analogies - they can be easily tailored to the specific needs of the individual.

\section{Future implications and conclusion}

From a clinical perspective, this study demonstrated that the application of analogy learning is feasible to facilitate improvements in walking performance in people with Parkinson's disease. Although the process of developing meaningful analogies might take time and require some creativity,24 once an appropriate analogy is found to correct a specific movement pattern, the concept is relatively easy to apply. In effect, the analogy used in the current study perhaps manages to provide some of the benefits of external and internal cues without their associated problems.61 There is no need for additional technology or information required for external cues, while potential problems with having to self-generate internal cues (increased cognitive load and an internal focus) are also potentially reduced.

While these preliminary results must be interpreted with caution, we conclude that analogies may improve walking performance in people with Parkinson's disease. Not only were these benefits found during a simple walking test, but improvements were also found under cognitive (counting backwards) and motor (carrying a tray) dual-task conditions. Future studies including control groups and appropriate sample sizes are needed to establish clinical effectiveness of analogy interventions.

\section{Key points}

- Analogy learning is a feasible learning strategy to facilitate walking performance in people with Parkinson's.

- The study brings implications for the delivery of instructions to facilitate walking performance in Parkinson's.

- Future studies, involving control groups and appropriate sample sizes, should establish the clinical effectiveness of analogy learning.

- Future studies could explore the use of different analogies on walking in Parkinson's.

\section{Acknowledgements}

We would like to thank Parkinson's UK for their support in recruiting participants and our participants for their involvement in this study. The authors also thank Professor Rich Masters for his comments on earlier drafts of this article. This study was financially supported by Stichting Alliantie Innovatie (Innovation Alliance Foundation), RAAK-international (Registration number: 2011-3- 33int), The Netherlands (MK, SB). VG is funded by the National Institute for Health Research (NIHR) Collaboration for Leadership in Applied Health Research and Care (CLAHRC) South West Peninsula at the Royal Devon and Exeter NHS Foundation Trust. The views expressed in this publication are those of the authors and not necessarily those of the NHS, the NIHR or the Department of Health in England. 



\section{CHAPTER 3}

\section{The immediate influence of implicit motor learning strategies on spatiotemporal gait parameters in stroke patients: a randomized within-subjects design}

Melanie Kleynen, Li-Juan Jie, Kyra Theunissen, Sascha MC Rasquin, Rich SW

Masters, Kenneth Meijer, Anna JHM Beurskens, Susy M Braun

Published as:

Kleynen M, Jie LJ, Theunissen K, Rasquin SM, Masters RS, Meijer K, Beurskens A, Braun S. The immediate influence of implicit motor learning strategies on spatiotemporal gait parameters in stroke patients: a randomized within-subjects design. Clin Rehabil. 2019;33(4):619-630.

(c) The Author(s) 2018. Article reuse guidelines: sagepub.com/journals-permissions DOI: 10.1177/0269215518816359 https://journals.sagepub.com/home/cre 


\begin{abstract}
Objectives: To investigate immediate changes in walking performance associated with three implicit motor learning strategies and to explore patient experiences of each strategy.

Design: Participants were randomly allocated to one of three implicit motor learning strategies. Within-group comparisons of spatiotemporal parameters at baseline and post strategy were performed.
\end{abstract}

Setting: Laboratory setting.

Subjects: A total of 56 community-dwelling post-stroke individuals.

Interventions: Implicit learning strategies were analogy instructions, environmental constraints and action observation. Different analogy instructions and environmental constraints were used to facilitate specific gait parameters. Within action observation, only videotaped gait was shown.

Main measures: Spatiotemporal measures (speed, step length, step width, step height) were recorded using Vicon 3D motion analysis. Patient experiences were assessed by questionnaire.

Results: At a group level, three of the four analogy instructions $(n=19)$ led to small but significant changes in speed $(d=0.088 \mathrm{~m} / \mathrm{s})$, step height (affected side $d=0.006 \mathrm{~m}$ ) and step width $(\mathrm{d}=-0.019 \mathrm{~m})$, and one environmental constraint $(n=17)$ led to significant changes in step width $(d=-0.040 \mathrm{~m})$. At an individual level, results showed wide variation in the magnitude of changes. Within action observation $(n=20)$, no significant changes were found. Overall, participants found it easy to use the different strategies and experienced some changes in their walking performance.

Conclusion: Analogy instructions and environmental constraints can lead to specific, immediate changes in the walking performance and were in general experienced as feasible by the participants. However, the response of an individual patient may vary quite considerably.

$24 \mid$ Immediate influence of implicit motor learning strategies 


\section{INTRODUCTION}

Improvement of walking ability after stroke is an essential and extensive part of rehabilitation, especially within physiotherapy.62 Therapists tend to support gait training by providing verbal instructions to facilitate optimal walking performance.6 However, many stroke survivors experience deficits in memory, attention, information processing and communication, which can hamper their ability to understand, process and remember verbal information or instructions during therapy and hence may hamper the walking training.63,64

Implicit motor learning strategies strive to minimize the use of verbal knowledge and, consequently, are thought to circumvent the need to explicitly understand, process and remember how to perform the motor task.8,65 Therefore, it has been hypothesized that implicit motor learning makes fewer demands on cognitive resources, especially working memory capacity.8,14,65

Literature describes different applications of implicit motor learning. On one hand, promising results were reported in patients with neurological conditions when analogy instructions, environmental constraints and action observation were used to promote (implicit) motor learning (see section 'Methods' for detailed information on these strategies).24,25,66-68 On the other hand, the provision of detailed verbal knowledge about the motor skill has been described as necessary to improve quality of motor performance in people after stroke.69,70 In walking, for example, detailed verbal instructions for improving gait could focus on spatiotemporal parameters, such as walking speed, step length and step width. However, it remains unclear whether implicit motor learning strategies aiming to minimize the use of verbal knowledge can also be used to influence specific spatiotemporal gait parameters.

The aim of this study was therefore to investigate immediate changes in walking performance associated with three implicit motor learning strategies and to explore patient experiences of each strategy.

\section{METHODS}

A short-term randomized design was used to explore immediate changes in walking performance when using three implicit learning strategies (Dutch Trial Register Number: NTR5510). The aim of the study was to investigate what changes in spatiotemporal parameters of the gait pattern are associated with each of these strategies. Therefore, spatiotemporal parameters of participants before and after the use of a strategy were compared for each strategy separately. 
People after stroke were invited to participate from December 2015 to December 2016. Ethical approval was provided by the local ethics committees (Zuyderland-Zuyd Ethics Committee 15N-153, Adelante MEC (MEC15-13)) and all participants provided informed consent. Measures were performed at one of two motion capture laboratories at Zuyd University of Applied Sciences or Maastricht University (The Netherlands). Participants were recruited from two rehabilitation centres, an outpatient clinic of a hospital and from seven physiotherapy private practices in the south of the Netherlands. Furthermore, a call for participation was placed in a local magazine for patients and informal caregivers. Inclusion criteria were: a stroke (>three months ago), capacity to walk independently with or without a walking aid over $10 \mathrm{~m}$ (with a self-selected gait speed $<1.2 \mathrm{~m} / \mathrm{s}$ ) and presence of hemiparesis (indicated by a score of $<100$ on the lower extremity part of the Motricity Index71 and a score <34 on the lower extremity part of the Brunnstrom Fugl-Meyer assessment72). Participants also needed to be able to visit one of the two motion capture laboratories and to have sufficient understanding of the Dutch language. Exclusion criteria were diagnosed impairments unrelated to stroke but with potential to influence gait pattern (e.g. severe osteoarthritis or amputation of the lower limb), diagnosed additional neurological impairments (e.g. Parkinson's disease).

Every participant was randomly allocated to one of the three strategies (analogy instructions, environmental constraint or action observation) by a researcher blind to the patient's characteristics, based on a computerized randomization schedule (block size 6). Randomization was performed in order to limit risk of selection bias; however, no between-group comparison was performed. The researchers who instructed the patients also performed the measurements and were therefore not blinded for the treatment allocation. The three motor learning strategies were applied using different conditions (Figure 1). Each condition targeted change in a specific spatiotemporal parameter (Supplemental Table 1). These conditions were developed based on piloting. Feasibility of the conditions was assessed by patient representatives.

\section{Analogy instructions}

An analogy uses understanding of a known concept or process to facilitate understanding or learning of a new concept or process. During motor learning, the complex structure of the 'to-be-learned' skill can be captured by an appropriate analogy, which is presented to the learner to aid performance of the movements.11,73 The idea is that the underlying verbal rules of the task are disguised within the analogy and the learner unintentionally (implicitly) employs these rules without gaining explicit knowledge. In this study, four different conditions (analogies) were used to influence primarily walking speed, step length, step width or step height. The analogies were presented to participants pictorially together with a brief instruction (Supplemental Table 1) before the walking trial. Participants were asked to use the analogy during walking. During the walking trial, no further instructions were provided. 


\section{Environmental constraints}

The environment can be constrained in early stages of the learning process to minimize performance errors.74 This limits the opportunity for error correction and consequently discourages the need for hypothesis testing that leads to explicit knowledge 75. In addition, an environment might guide the learner towards a certain movement pattern, without the need for verbal instructions.19 In this study, three different conditions (constraints) were used to influence primarily speed, step length or step width (Supplemental Table 1). To influence step length, horizontal black and white stripes were projected on the floor, creating a zebra crossing. Participants were instructed to step on the projected stripes. To influence walking speed, a horizontal bar that moved at a constant speed was projected onto the walkway. Participants were instructed to follow the bar without catching up to it. The distance between the stripes and speed of the bar was incrementally increased by $5 \%$, $10 \%$ and $15 \%$ of baseline performance. To influence step width, a narrow beam was projected onto the floor and participants were instructed to only step on the beam as they walked (decrease step width). The width of the beam was incrementally reduced to be $5 \%$, $10 \%$ and $15 \%$ narrower than step width at baseline. The participants received brief instructions (Supplemental Table 1) before the walking trial and the projected stripes remained visible during the walking trial.

\section{Action observation}

In action observation, the amount of verbal instructions can be limited using modelling (demonstration) and video observation.20 In this study, action observation was applied using only one condition (observation of a short video clip) and the strategy did not target a specific gait parameter. Different video clips of a healthy older male or female walking with different walking aids (e.g. stick, rollator) were used. The video clip that was viewed was chosen based on the gender of the participant and type of walking aid used. The person on the video was shown from a frontal view and a side-view. Participants watched the video and were instructed to try to imitate the walking of the person in the video. During walking, no further instructions were provided.

In all three groups, each condition was repeated three times and a minimum of nine complete strides per condition were included in the analysis. Participants in the analogy group therefore performed 12 trials ( 3 repetitions of 4 conditions), whereas participants in the environmental constraint group performed 9 trials ( 3 repetitions of 3 conditions) and participants in the observational group performed 3 trials ( 3 repetitions of one conditions). Following baseline measurement and each condition, there was a short break in order to limit fatigue (Figure 1). In analogy instructions and environmental constraints, the order of the conditions was counterbalanced (Latin square) in order to offset the possibility of carry-over effects. 


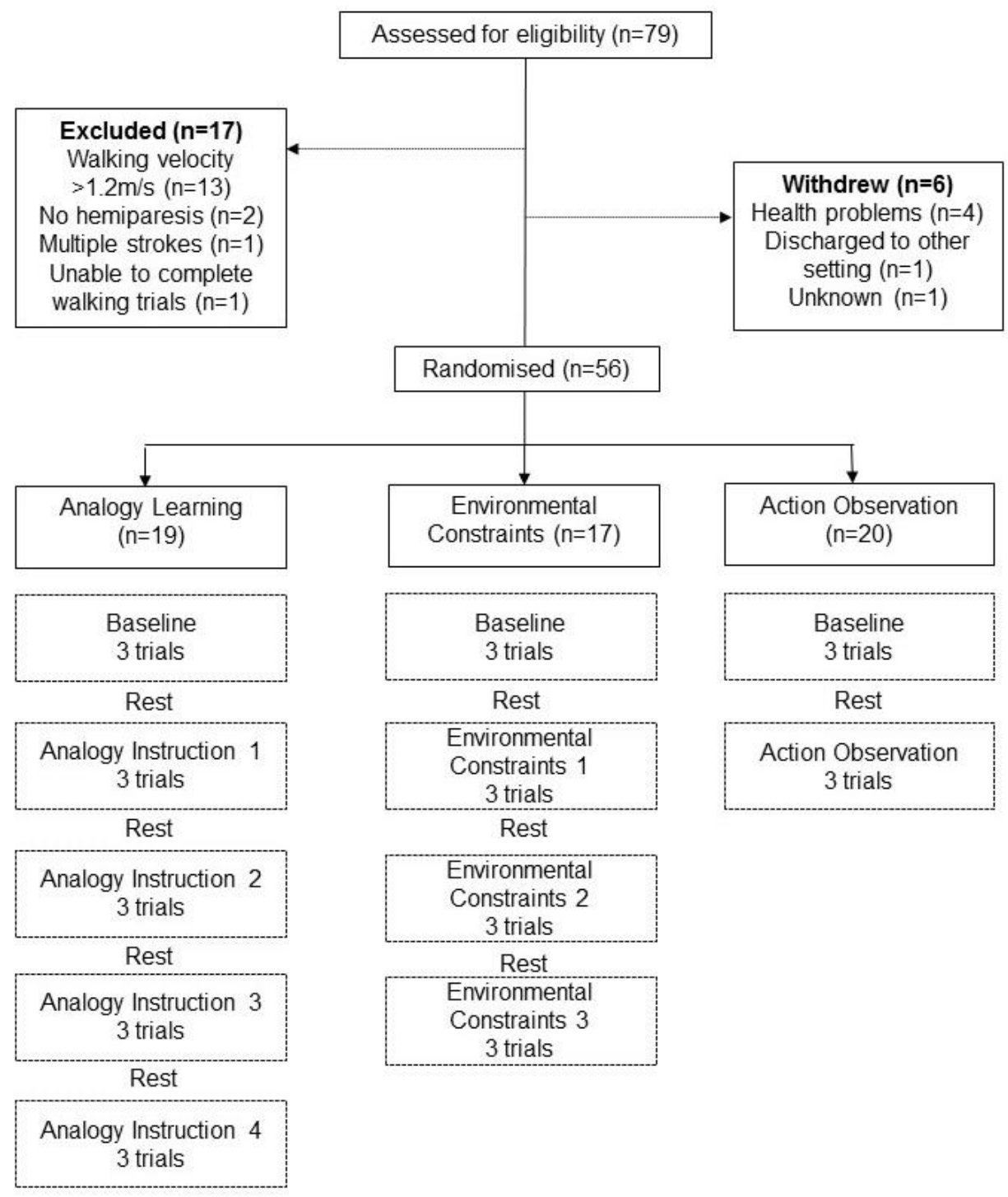

Figure 1. Flow of participants and overview methods.

We collected the following background data: Motricity Index (voluntary movement activity and maximum muscle strength),71 Berg Balance Scale (static balance and fall risk)76, Rivermead mobility index (mobility disability) 77 and Fugl-Meyer Assessment of the lower extremity (ability to make movements outside the synergetic patterns).72 Cognitive functioning was investigated using the Montreal Cognitive Assessment (detection of mild cognitive problems, scores $>26$ are considered as normal), 78 the subtest 'news story' of the Rivermead Behavioural Memory Test (memory function) 79 and the D2 attention test (attention span and concentration).80 Performance on the Rivermead

$\mathbf{2 8}$ | Immediate influence of implicit motor learning strategies 
Behavioural Memory Test and the D2 is presented as percentile scores normalized for age, gender (D2, Rivermead Behavioural Memory Test) and educational level (Rivermead Behavioural Memory Test).

The following spatiotemporal parameters were collected in order to assess adaptations to the instructions: speed, step length, step width and step height. The gait parameters (Table 1) were calculated using a custom MATLAB script (version 2012a, The MathWorks, USA). Gait events were determined using an algorithm consistent with Zeni et als1 Asymmetry ratios of step lengths and swing time were calculated according to Awad et al.82 A value of 0.5 reflects perfect symmetry.

Table 1. Operationalization of the spatiotemporal parameters.

\begin{tabular}{|c|c|c|}
\hline Variable & Markers & Calculation \\
\hline Walking speed & $\begin{array}{l}\text { Mean position of the } 4 \text { hip markers } \\
\text { to estimate the centre of mass }\end{array}$ & $\begin{array}{l}\text { Dividing the distance walked by the ambulation } \\
\text { time. }\end{array}$ \\
\hline Step length & Heel markers & Distance between heel markers at heel strike. \\
\hline Step height & Ankle marker & $\begin{array}{l}\text { Difference in minimal and maximal height } \\
\text { within two consecutive heel strikes of the same } \\
\text { leg within one step. }\end{array}$ \\
\hline Step width & Ankle markers & $\begin{array}{l}\text { Distance between two ankle markers at double } \\
\text { contact. }\end{array}$ \\
\hline $\begin{array}{l}\text { Step length } \\
\text { asymmetry }\end{array}$ & - & $\begin{array}{l}\text { Larger step length/(Larger step length }+ \text { Smaller } \\
\text { step length) }\end{array}$ \\
\hline $\begin{array}{l}\text { Swing time } \\
\text { asymmetry }\end{array}$ & - & $\begin{array}{l}\text { Longer swing time/(Longer swing time + } \\
\text { shorter swing time) }\end{array}$ \\
\hline
\end{tabular}

The participants' experiences of the strategies were explored using a self-developed questionnaire to gain insight into opinions, feasibility and perceived improvements in walking performance. Responses were recorded using multiple-choice options and free comments. It has been shown that implicit motor learners tend to report fewer verbal rules about their movements during performance than explicit motor learners.83 After completion of the session, participants were therefore asked to report in detail all rules and techniques they were aware of, or used, during the walking trials. A rule was defined as any statement that contained at least one movement or position of a limb or joint, the velocity of a limb moving, an angle or directions of a joint, placement of the walking aid, changes in the use of the walking aid or changes in step characteristics (bigger steps, wider steps, etc.).

Data on spatiotemporal parameters were collected with a Vicon motion analysis system (Vicon Motion Systems Ltd, Oxford, UK), consisting of eight infrared motion capture cameras running at $200 \mathrm{~Hz}$. The cameras were spaced around a $10 \mathrm{~m}$ walkway (Laboratory 1) or a $12 \mathrm{~m}$ walkway (Laboratory 2 ). A total of 35 reflective markers (14mm) were affixed to participants with adhesive tape according to the Plug-in Gait full body model. Data were 
processed using Vicon Nexus software version 1.8.5. In a pilot study, the betweenlaboratory reliability of data was shown to be good to excellent for the spatiotemporal parameters measured (intraclass correlation coefficients (ICCs) between 0.84-0.96, data available on request).

All statistical analyses were conducted in SPSS version 24. Population characteristics and background data are presented using mean values and SD per strategy and for the entire group of participants. Discrete variables are presented using absolute numbers. Statistical testing was used to examine differences between baseline performance and performance during the condition, using a within-group comparison for each of the three strategies (analogy instructions, environmental constraints and action observation) separately. Repeated-measures analysis of variance (ANOVA) and planned contrasts (baseline performances compared to each condition) were used to investigate the analogy instructions over five time points (baseline and four different conditions), the environmental constraints over four time points (baseline and three different conditions) and the action observation over two time points. Non-parametric tests were performed if there was violation of the normality assumption (Friedman's ANOVA combined with Wilcoxon signed-rank tests for multiple comparison and Bonferonni correction). An alpha level of 0.05 was adopted for all tests. Percentage changes from baseline performance for the main outcome parameters are presented using bar charts.

Besides statistical testing, results were analysed in terms of clinical relevance. Individual changes in walking speed were assessed using the clinically important change, which is $0.175 \mathrm{~m} / \mathrm{s}$ according to Fulk et al84. To our knowledge, the clinically important change has not been reported for the other spatiotemporal parameters. Participants' evaluations were analysed descriptively and quotes are used to illustrate their experiences.

30 | Immediate influence of implicit motor learning strategies 


\section{RESULTS}

A total of 56 participants completed the study (Figure 1). Table 2 presents the demographic information of the participants and the background. In Table 3, mean values for each gait parameter are presented together with results of the statistical tests.

On a group level, three of the four applied analogies led to small but significant changes in walking speed, step height (affected side) and step width. The 'small bridge' instruction (Supplemental Table 1) resulted in a significant mean decrease in step width, accompanied by a decrease in speed and step length (both affected and non-affected legs). The 'traffic light' instruction resulted in the intended increase in walking speed, accompanied by an increase in step length (both affected and non-affected legs) and an increase in step height (both affected and non-affected legs). Step height of both legs and step width increased after participants received the 'deep snow' instruction; however, speed decreased.

In the environmental constraints strategy, only the 'narrow beam' led to the intended change in step width, but speed, step length (affected and non-affected legs) and step height (affected leg only) decreased in this strategy.

In the action observation strategy, walking speed in general decreased. No significant changes were evident for the asymmetry ratios.

In the Supplemental Tables 1-3d, percentage change from baseline in the different conditions is presented visually as bar charts. A broad individual range of changes is apparent (indicated by the error bars). There were participants who responded to the analogy instructions or the environmental constraints with the indented changes in their walking performance. For example, seven participants $(n=3$ environmental constraints, $n=4$ analogy instructions) increased their walking speed by greater than $0.175 \mathrm{~m} / \mathrm{s}$, exceeding the clinically important change (maximum increase was $0.66 \mathrm{~m} / \mathrm{s}$ ). These participants on average dis- played higher baseline walking speed (mean (SD) $0.72 \mathrm{~m} / \mathrm{s}$ (0.18)), slightly better functioning of the affected leg (mean (SD) Motricity Index: 71.4 (8.0) and Brunnstrom Fugl-Meyer assessment: 28.6 (4.3)) and superior balance (mean (SD) Berg Balance Scale: 47.6 (5.8)), compared to the mean of all participants. In other participants, the intended spatiotemporal parameter did not change or even changed in the opposite direction (e.g. decrease in walking speed or step length). 
Table 2. Demographic information of participants.

\begin{tabular}{|c|c|c|c|c|}
\hline & $\begin{array}{l}\text { Analogy } \\
\text { instruction } \\
(n=19)\end{array}$ & $\begin{array}{l}\text { Environmental } \\
\text { constraints } \\
(n=17)\end{array}$ & $\begin{array}{l}\text { Action } \\
\text { observation } \\
(n=20) \\
\end{array}$ & $\begin{array}{l}\text { All } \\
\text { participants } \\
(N=56) \\
\end{array}$ \\
\hline Age in years, mean (SD) & $67.0(11.9)$ & $61.1(11.9)$ & $63.9(12.5)$ & $64.1(12.0)$ \\
\hline \multicolumn{5}{|l|}{ Gender, $n$} \\
\hline Male & 10 & 11 & 11 & 32 \\
\hline Female & 9 & 6 & 9 & 24 \\
\hline Length in cm, mean (SD) & $170.4(8.8)$ & $174.5(7.2)$ & $170.1(10.7)$ & $171.5(9.1)$ \\
\hline Weight in kg, mean (SD) & $80.7(19.3)$ & $78.9(13.7)$ & $77.9(15.8)$ & $79.15(16.2)$ \\
\hline \multicolumn{5}{|l|}{ Side of the stroke, $n$} \\
\hline Left & 10 & 8 & 10 & 28 \\
\hline Right & 9 & 9 & 10 & 28 \\
\hline $\begin{array}{l}\text { Time post stroke in } \\
\text { months, mean (SD) }\end{array}$ & $87.2(137.5)$ & $89.4(84.7)$ & $61.8(57.7)$ & $78.8(97.9)$ \\
\hline \multicolumn{5}{|l|}{ Walking aid, $n$} \\
\hline None & 4 & 5 & 7 & 16 \\
\hline Cane & 6 & 7 & 7 & 20 \\
\hline Quad cane & 3 & 4 & 1 & 8 \\
\hline Rollator & 6 & 1 & 3 & 10 \\
\hline Crutch & - & - & 2 & 2 \\
\hline \multicolumn{5}{|l|}{ Educational Level, $n$} \\
\hline $\begin{array}{l}\text { Elementary } \\
\text { education }\end{array}$ & 4 & - & - & 4 \\
\hline Secondary education & - & 8 & 11 & 28 \\
\hline Vocational training & 9 & 4 & 4 & 8 \\
\hline University & 6 & 5 & 5 & 16 \\
\hline \multicolumn{5}{|l|}{$\begin{array}{l}\text { Physical functioning, } \\
\text { mean (SD) }\end{array}$} \\
\hline BBS $(0-56)$ & $43.7(10.9)$ & $42.0(8.9)$ & $46.2(11.0)$ & $44.1(10.3)$ \\
\hline MI total score $(0-200)$ & $122.1(37.7)$ & $98.2(43.7)$ & $109.1(40.1)$ & $110.2(40.9)$ \\
\hline Lower extremity $(0-100)$ & $63.7(15.7)$ & $56.1(19.5)$ & $57.8(15.1)$ & $59.3(16.8)$ \\
\hline Upper extremity $(0-100)$ & $58.4(28.7)$ & $42.1(30.0)$ & $51.3(28.5)$ & $50.9(29.2)$ \\
\hline FMA $(0-34)(n=54) a$ & $23.8(2.1)$ & $19.9(7.9)$ & $22.1(7.9)$ & $22.0(7.2)$ \\
\hline RMI (0-15) & $11.8(2.1)$ & $12.1(1.9)$ & $11.85(2.8)$ & $11.93(2.3)$ \\
\hline \multicolumn{5}{|l|}{$\begin{array}{l}\text { Cognitive functioning, } \\
\text { mean (SD) }\end{array}$} \\
\hline D2 & $n=16$ & $n=17$ & $n=19$ & $n=52 \mathrm{~b}$ \\
\hline TN-F & $45.4(12.3)$ & $19.9(22.3)$ & $44.7(13.0)$ & $45.79(12.04)$ \\
\hline $\mathrm{CP}$ & $45.8(11.8)$ & $27.8(28.0)$ & $44.5(14.3)$ & $46.21(12.04)$ \\
\hline RMBT & $n=17$ & $n=17$ & $n=19$ & $(n=53)_{\mathrm{c}}$ \\
\hline immediate recall & $27.7(26.6)$ & $19.9(22.3)$ & $19.8(26.7)$ & $22.4(25.1)$ \\
\hline delayed recall & $36.8(33.2)$ & $27.8(28.0)$ & $26.6(23.3)$ & $30.3(28.1)$ \\
\hline $\operatorname{MOCA}(0-30)$ & $22.4(5.5)$ & $24.5(3.9)$ & $23.5(4.7)$ & $23.4(4.8)$ \\
\hline
\end{tabular}

RBMT: Rivermead Behavioural Memory Testing; MOCA: Montreal Cognitive Assessment; BBS: Berg balance scale; MI: Motricity Index; FMA: Fugl-Meyer Assessment; RMI: Rivermead Mobility Index; TN-F: the number of all errors relative to the total number of items processed (measure of precision and thoroughness); CP: number of correctly marked characters minus the number of incorrectly marked characters (measure of attention span and concentration ability).

a $n=2$ missing (missed appointment $(n=1)$; test not correct $(n=1)$ ).

$\mathrm{b} n=4$ missing (did not understand instructions $(\mathrm{n}=2)$; not able to read letters $(n=1)$; missed appointment $(n=1))$.

cn $=3$ missing (did not understand instructions). 
Variation in step length change in the 'zebra crossing' condition (environmental constraint) and the 'footprints in the sand' condition (analogy instruction) are particularly broad. For instance, two participants were able to increase the step length of their nonaffected leg from 2.4 to $14.9 \mathrm{~cm}$ (analogy instructions group) and from 2.7 to $19.5 \mathrm{~cm}$ (environmental constraints group). In both cases, this resulted in better step-through gait (baseline step length asymmetry 0.94 and 0.90 , post/during-condition asymmetry 0.69 and $0.60)$.

Overall, participants found it relatively easy to walk during the different conditions and did not report a need to think much more than usual (Supplemental Table 2). However, some instructions reminded people of difficult situations in daily life. For example, after using the walking in 'deep snow' analogy, one participant stated that 'in real life I cannot walk through snow with my wheeled rollator', and after using the traffic light analogy, another participant stated that 'in real life I never make it on time to the other side of the road'. In general, participants experienced some change in their walking performance. In the action observation strategy, people frequently reported that they found themselves attending to their 'arm swing' and to 'walking more upright'. 


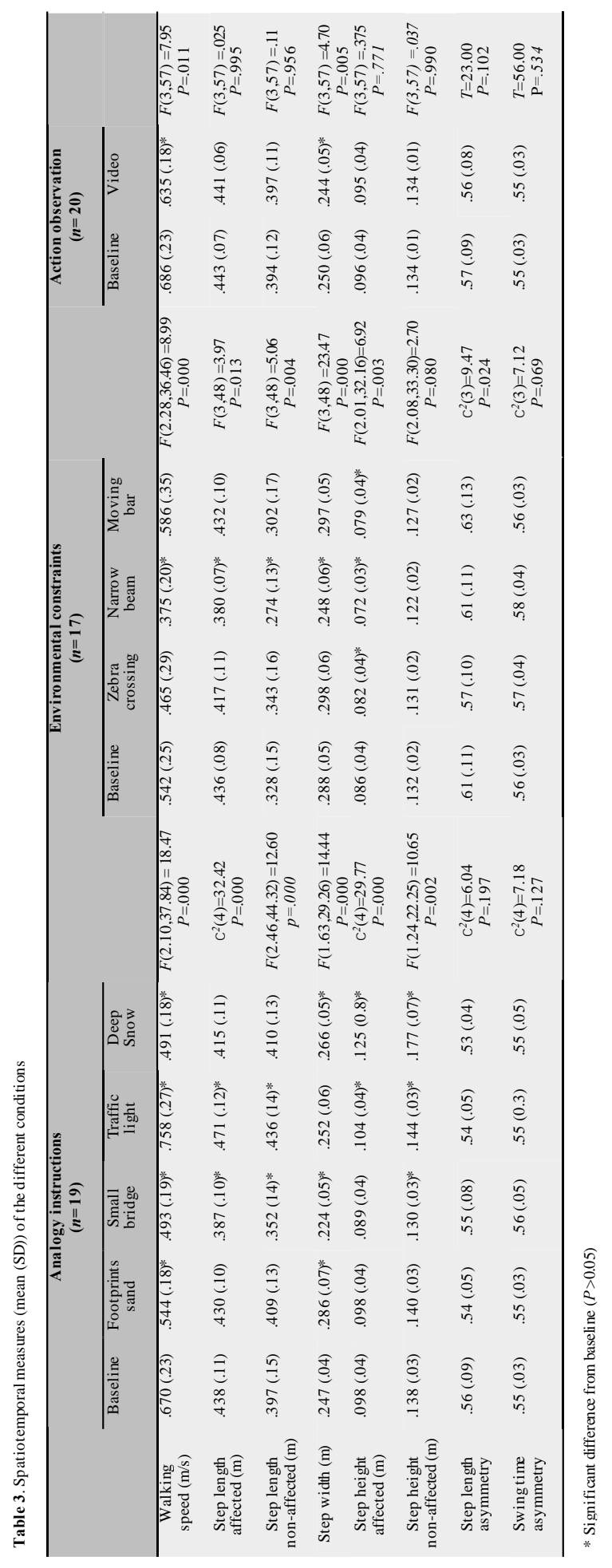

34 | Immediate influence of implicit motor learning strategies 


\section{DISCUSSION}

The data from this study suggest that in general analogy instructions and environmental constraints can be used to facilitate specific, immediate changes in spatiotemporal gait parameters without providing detailed verbal knowledge. At a group level, three of the four applied analogies led to small but significant changes in walking speed, step height (affected side) and step width. Environmental constraints led to significant changes in step width. For the action observation strategy, no significant changes were found. The use of analogy instructions or environmental constraints changed walking performance in some participants even beyond clinically relevant changes, at least temporarily. However, individual results showed wide variation in the magnitude of changes.

This large individual variation may be explained by the fact that the conditions were predefined rather than tailored to the needs of individual participants. For example, in analogy learning, some participants reported that the situations portrayed in the analogies were meaningful for them, whereas other participants stated that these situations were difficult or uncomfortable in daily life. The meaningfulness of the analogy may have influenced the response and consequently the outcome. 85 Within the environmental constraints group, the wide variation within the 'zebra crossing' condition may be explained by large performance differences at baseline. Some participants already walked relatively symmetrically with a step-through gait pattern, whereas others adopted a more asymmetric step-to gait pattern and may therefore have shown greater improvements in step length and step length asymmetry. Similarly, a ceiling effect might have occurred for participants who already had a small ('normal') step width at baseline, causing any further decrease to culminate in an unnatural walking pattern. In action observation, participants often reported that they paid attention to aspects of gait that were either relevant to their own specific walking problem (e.g. stability of the knee joint) or general aspects of gait, such as arm swing and walking more up-right. This variation in interpretation of the instruction might explain the absence of significant specific changes, at least on a group level. In general, these findings suggest that there is a need for motor learning strategies to be tailored to the individual gait pattern and personal preferences and experiences of the patients. The need for tailoring motor learning strategies was recently also confirmed by a randomized controlled trial comparing the effects of internal and external focus instructions.86

Gait is a multivariate phenomenon with a pattern across the several parameters, and it is well known that a change of one gait parameter is generally accompanied by changes of other gait parameters. 87 A strength of the study is that a broad set of measures was used in order to measure the overall pattern of change. We were able to detect which additional spatiotemporal parameters might change alongside the primary parameter of interest. For example, an increase of walking speed in the 'traffic light' analogy also led to 
accompanied changes in step length and height. Furthermore, a decrease in speed was observed in several conditions. It might be that patients require time to fully assimilate a new motor learning strategy and slow down initially in order to focus on the primary aim (e.g. step length).68

Another strength of the study is the relatively heterogeneous sample of patients after stroke that was included, especially regarding their cognitive abilities. It is remarkable that all included patients were able to complete the applied intervention even those who were not capable of completing the cognitive tests because of severe cognitive or communicative problems. Regardless of cognitive and communicative impairments, most participants found it easy to use the different strategies.

Next to this strength, some limitations should be considered when interpreting the present results. First, relatively permanent changes in motor behaviour, representing learning, are typically only convincingly evidenced by delayed retention tests or during transfer of a motor skill.88 The absence of such follow-up testing is a clear limitation of the study. Second, the results of the statistical testing on a group level should be interpreted with caution, because there is a chance of bias as a result of multiple testing. Third, the included sample size did not allow subgroup analysis. For instance, baseline walking speed, balance or motor function might have influenced physical ability to respond to the motor learning strategy. 89,90

Besides these limitations, physiotherapists and other healthcare professionals involved in motor learning of patients can learn from this study that specific changes in spatiotemporal measures of gait can occur if analogy instructions and environmental constraints are used. The strategies explored in this study were applied using only a single instruction. They might be an efficient therapy option, especially in participants who experience problems with understanding and processing more detailed verbal instructions. Therapists should be aware that using these motor learning strategies does not necessarily prevent patients from consciously controlling their gait and that changing a specific parameter will most likely result in concomitant changes (improvement or deterioration) of additional parameters.

Future research should investigate whether implicit motor learning strategies lead to changes in gait performance that are stable over several sessions, in retention and in realworld overground walking situations. Future studies should also directly compare the effects of implicit motor learning to an explicit control intervention, because in stroke evidence for the superiority of implicit motor learning is inconclusive.91 This study provides evidence that tailoring motor learning strategies towards individuals' abilities and preferences is important. In practice, therapists seem to take many factors into account when shaping motor learning in practice, which results in highly individualized approaches 92 that cannot be captured within one research paradigm. In order to unravel 
the complexity of motor learning, different qualitative and quantitative research designs are needed and should probably be combined with systematic observations of which strategy fits the patient's abilities and preferences within clinical practice.

\section{Clinical Messages}

- Analogy instructions and environmental constraints led to immediate changes in walking performance.

- For action observation, no significant changes were found.

- Individual results showed wide variation in the magnitude of changes.

- The researched strategies seem feasible but need to be tailored to the individual gait problem and preferences of the patients.

\section{Acknowledgements}

The authors would like to thank Nathalie Sieben, Else de Bont and Anja Minheere for their valuable support of the entire study as client representatives, Elmar Kal and John van der Kamp for their input and suggestions, and Wouter Bijnens for his support with the data processing.

\section{Declaration of Conflicting Interests}

The author(s) declared no potential conflicts of interest with respect to the research, authorship and/or publication of this article.

\section{Funding}

The author(s) disclosed receipt of the following financial support for the research, authorship and/or publication of this article: This work was supported by Nationaal Regieorgaan Praktijkgericht Onderzoek SIA (RAAKPRO; grant number 2014-0149PRO). 


\section{Supplemental material}

Supplemental Table 1. Overview conditions applied within analogy instructions and environmental constrains.

\begin{tabular}{lll}
\hline Intended change Analogy instructions with picture & $\begin{array}{l}\text { Environmental constrains with } \\
\text { instructions }\end{array}$ \\
\hline $\begin{array}{l}\text { Increased step } \\
\text { length }\end{array}$ & "Walk as if you follow footprints in the \\
sand" & why to alternately step on the black and
\end{tabular}

38 | Immediate influence of implicit motor learning strategies 
Supplemental Table 2. Overview results of participants' experiences

\begin{tabular}{|c|c|c|c|}
\hline & Analogy instructions & $\begin{array}{l}\text { Environmental } \\
\text { constrains }\end{array}$ & Action observation \\
\hline $\begin{array}{l}\text { 1. How difficult or easy did } \\
\text { you find walking with the } \\
\text { instructions? } \\
\text { ( } 1 \text { = very difficult, } 10=\text { not } \\
\text { difficult) }\end{array}$ & $7.6(2.2)$ & $6.2(3)$ & $8.0(1.5)$ \\
\hline $\begin{array}{l}\text { 2. Which instruction did } \\
\text { you find easiest to } \\
\text { perform? }\end{array}$ & $\begin{array}{l}\text { Traffic light: } 52.6 \% \\
(n=10) \\
\text { Deep snow: } 15.8 \% \\
(n=3) \\
\text { Footprints sand: } 5.3 \% \\
(n=1) \\
\text { Small bridge: } 0 \% \\
\text { ( } n=0) \\
\text { No preference: } 21.1 \% \\
(n=4) \\
\text { Missing: } 5.3 \%(n=1)\end{array}$ & $\begin{array}{l}\text { Zebra crossing: } 47.1 \% \\
(n=8) \\
\text { Narrow beam: } 29.4 \% \\
(n=5) \\
\text { Moving bar: } 23.5 \% \\
(n=4)\end{array}$ & N/A \\
\hline $\begin{array}{l}\text { 4. Did you need to think a } \\
\text { lot while walking? } \\
(1=\text { not at all, } 10=\text { need to } \\
\text { think a lot })\end{array}$ & $2.9(2.3)$ & $3.5(2.2)$ & $3.0(2.3)$ \\
\hline $\begin{array}{l}\text { 5. Do you think your gait } \\
\text { changed? } \\
(1=\text { no change, } 10=a \text { lot } \\
\text { of change })\end{array}$ & $\begin{array}{l}\text { Deep snow } 6.4(1.3) \\
\text { Footprints sand } 4.7 \\
(2.3) \\
\text { Traffic light } 4.2(2.8) \\
\text { Small bridge } 5.3(2.4)\end{array}$ & $\begin{array}{l}\text { Zebra crossing } 4.0 \\
(3.0) \\
\text { Narrow beam } 5.3(3.3) \\
\text { Moving bar } 3.8(2.8)\end{array}$ & $3.5(2.9)$ \\
\hline 6. Number of explicit rules & $\begin{array}{l}0 \text { rules: } 15.8 \%(n=3) \\
1-2 \text { rules: } 63.1 \% \\
\text { ( } n=12) \\
3-4 \text { rules: } 21.1 \%(n=4)\end{array}$ & $\begin{array}{l}0 \text { rules: } 23.5 \%(n=4) \\
\text { 1-2 rules: } 35.3 \%(n=6) \\
3-4 \text { rules: } 41.1 \%(n=7)\end{array}$ & $\begin{array}{l}0 \text { rules: } 30 \%(n=6) \\
1-2 \text { rules: } 50 \%(n=10) \\
3-4 \text { rules: } 20 \%(n=4)\end{array}$ \\
\hline
\end{tabular}


Supplemental Figure 1. Mean \% changes from baseline in the action observation condition.

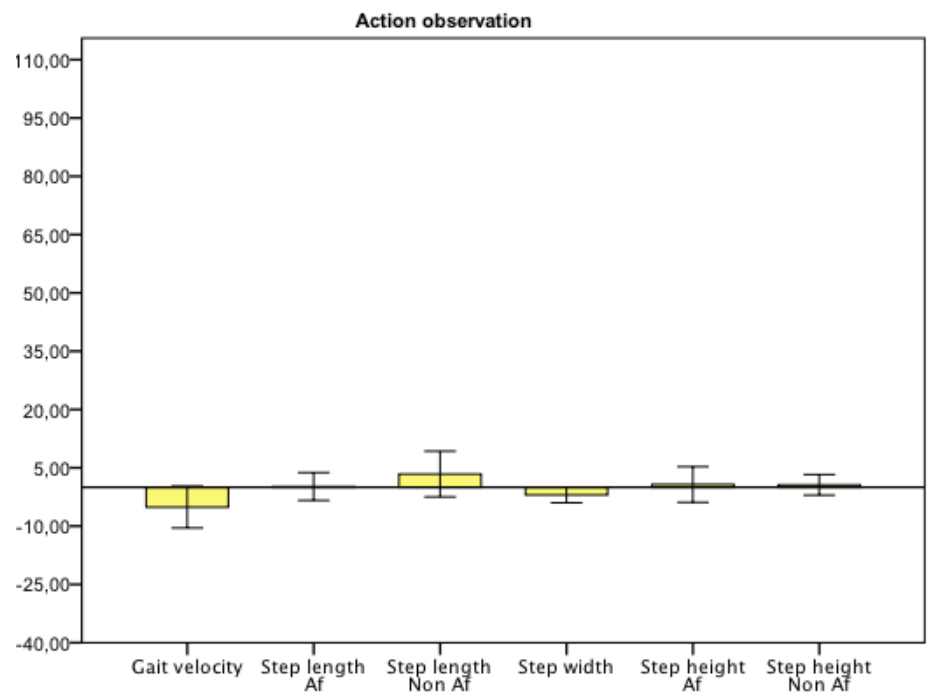

Supplemental Figure 2a. Mean \% changes from baseline in the environmental constraints (zebra crossing) condition.

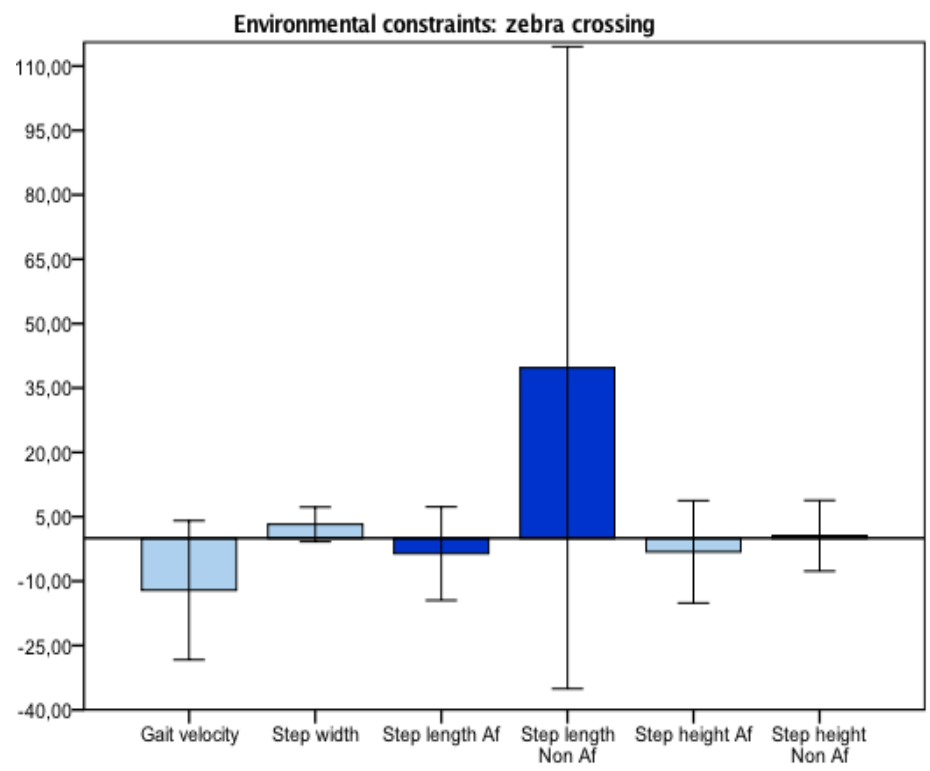

$40 \mid$ Immediate influence of implicit motor learning strategies 
Supplemental Figure 2b. Mean \% changes from baseline in the environmental constraints (narrow beam) condition.

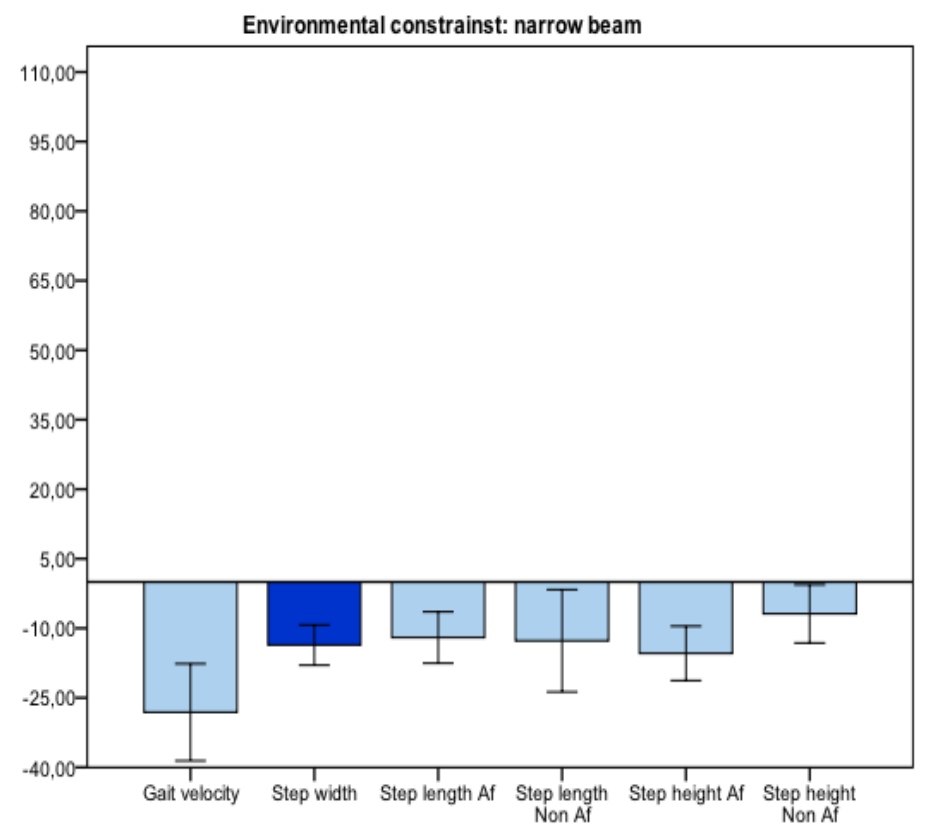

Supplemental Figure 2c. Mean \% changes from baseline in the environmental constraints (moving bar) condition.

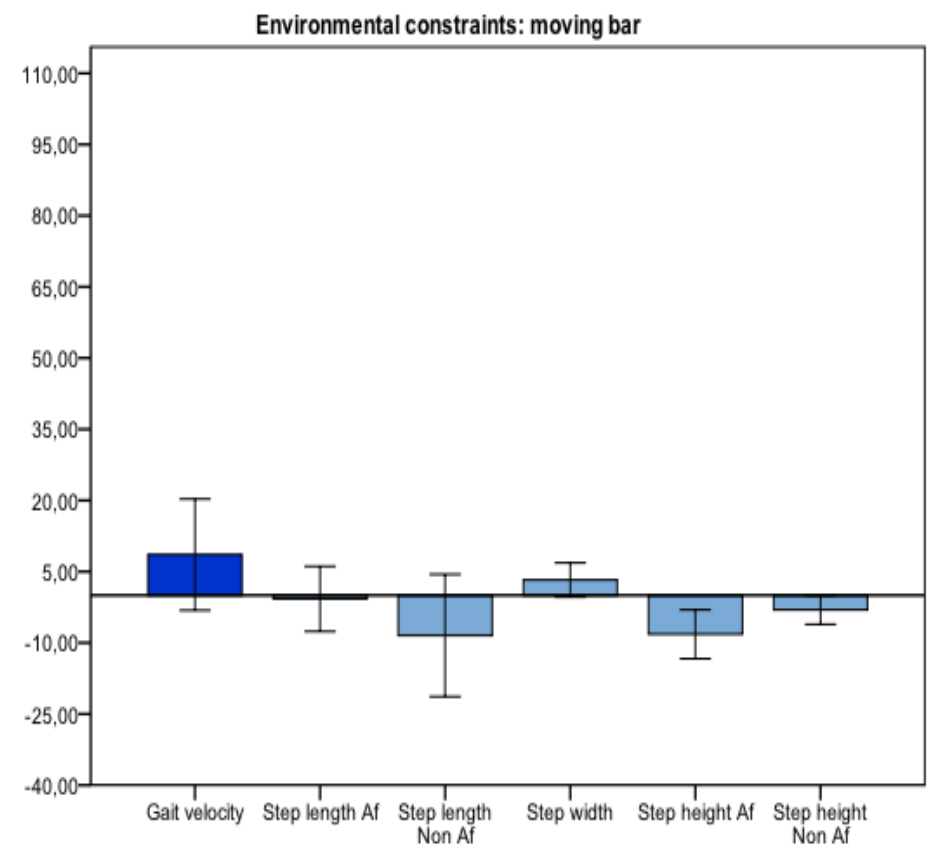


Supplemental Figure 3a. Mean \% changes from baseline in the analogy instruction (footprints sand) condition.

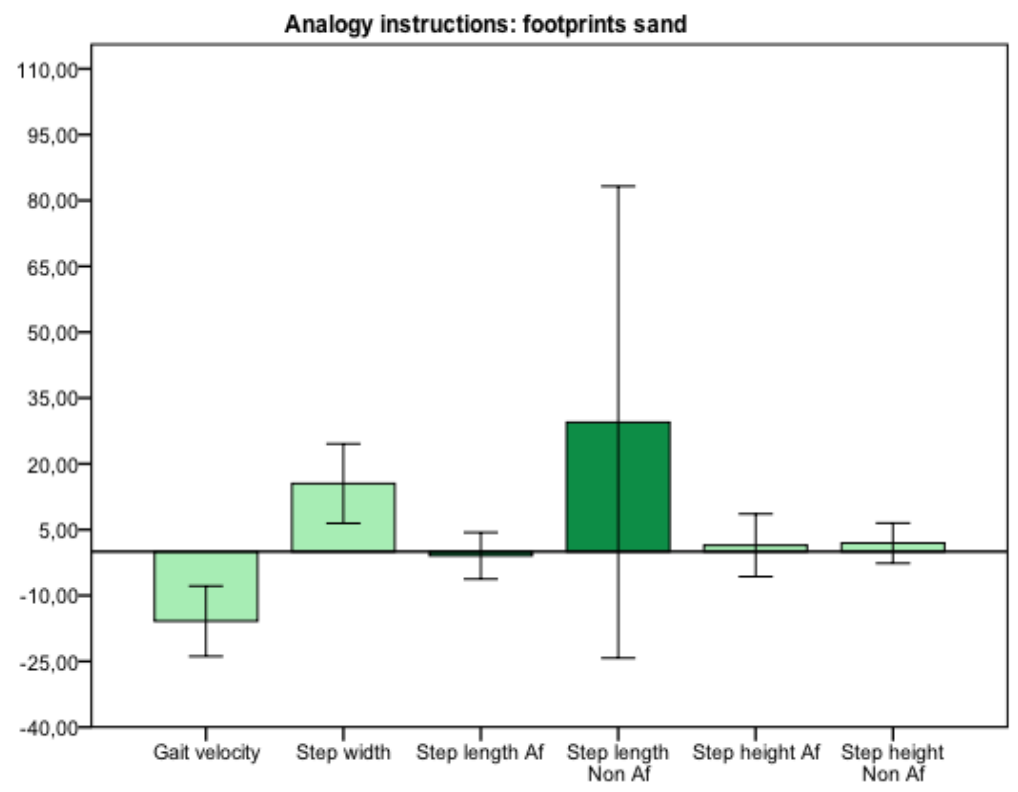

Supplemental Figure 3b. Mean \% changes from baseline in the analogy instruction (small bridge) condition.

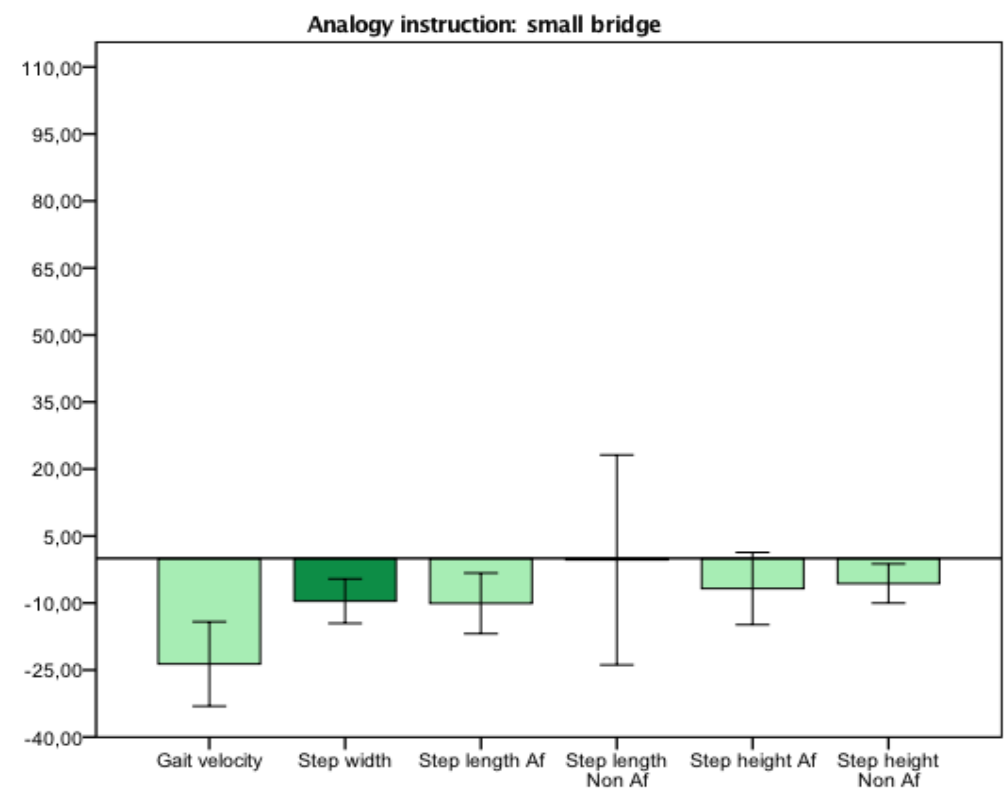

$42 \mid$ Immediate influence of implicit motor learning strategies 
Supplemental Figure 3c. Mean \% changes from baseline in the analogy instruction (traffic light) condition.

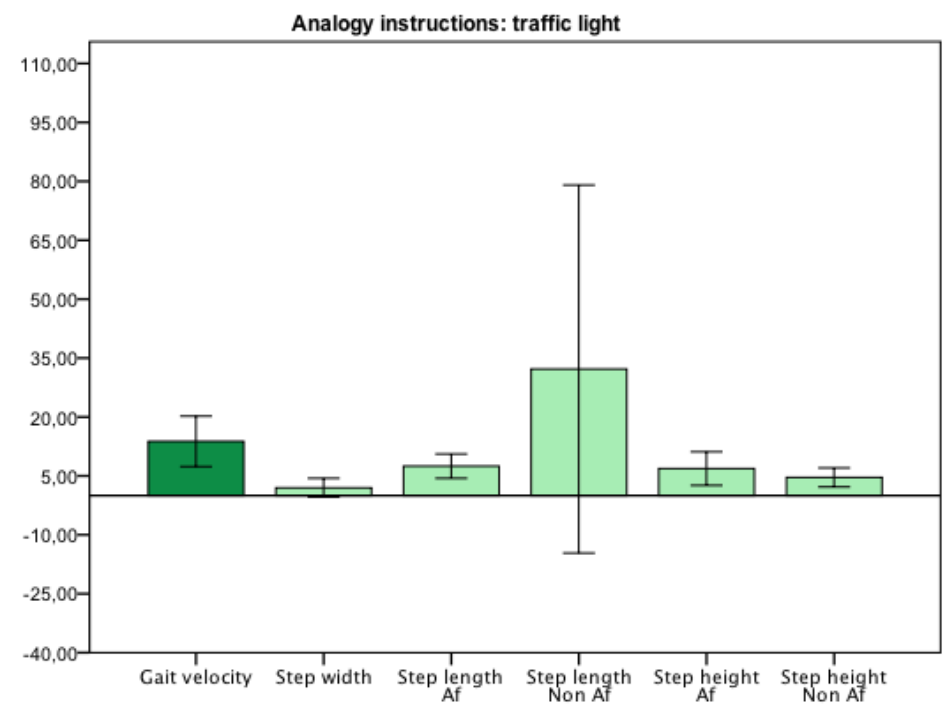

Supplemental Figure 3d. Mean \% changes from baseline in the analogy instruction (deep snow) condition.

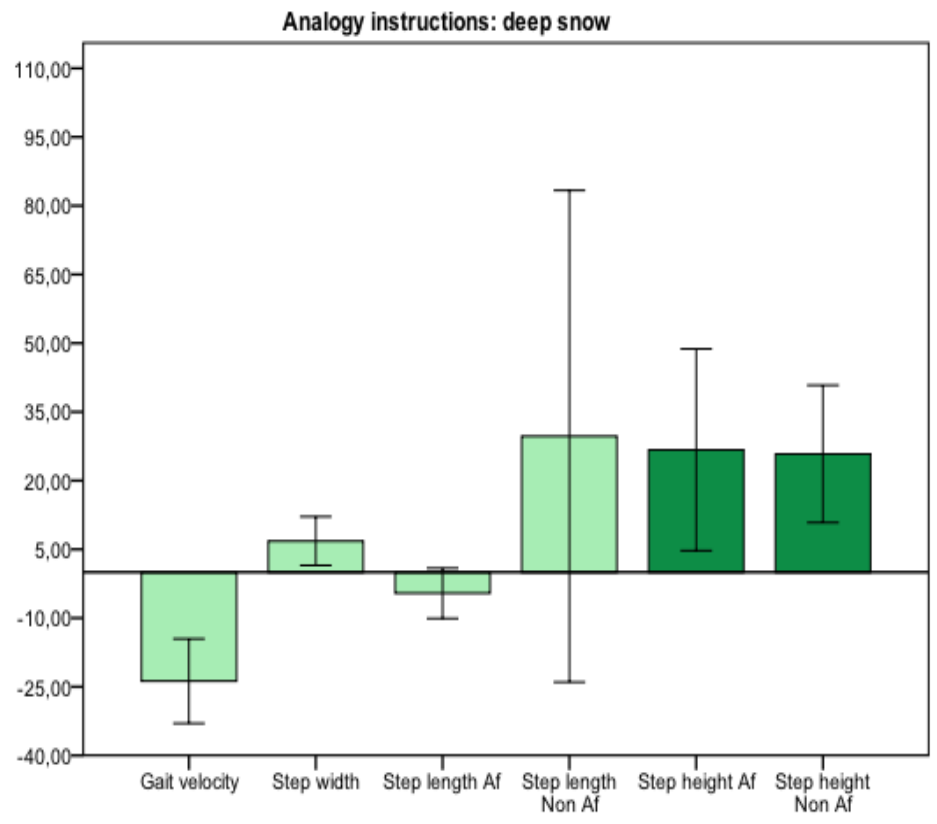





\section{CHAPTER 4}

\section{The Effects of Implicit and Explicit Motor Learning in Gait Rehabilitation of People After Stroke: Protocol for a Randomized Controlled Trial}

Li-Juan Jie, Melanie Kleynen, Kenneth Meijer, Anna JHM Beurskens, Susy M Braun

\section{Published as:}

Jie, L. J., Kleynen, M., Meijer, K., Beurskens, A., \& Braun, S. (2018). The Effects of Implicit and Explicit Motor Learning in Gait Rehabilitation of People After Stroke: Protocol for a Randomized Controlled Trial. JMIR Res Protoc, 7(5), e142-e142. doi: 10.2196/resprot.9595

(C) 2018. This manuscript version is made available under the CC-BY-NC-ND 4.0 license. http://creativecommons.org/licenses/by-nc-nd/4.0/ 


\begin{abstract}
Background: A significant part of neurological rehabilitation focuses on facilitating the learning of motor skills. Training can adopt either (more) explicit or (more) implicit forms of motor learning. Gait is one of the most practiced motor skills within rehabilitation in people after stroke because it is an important criterion for discharge and requirement for functioning at home.
\end{abstract}

Objective: The aim of this study was to describe the design of a randomized controlled study assessing the effects of implicit motor learning compared with the explicit motor learning in gait rehabilitation of people suffering from stroke.

Methods: The study adopts a randomized, controlled, single-blinded study design. People after stroke will be eligible for participation when they are in the chronic stage of recovery (>6 months after stroke), would like to improve walking performance, have a slow walking speed $(<1 \mathrm{~m} / \mathrm{s})$, can communicate in Dutch, and complete a 3-stage command. People will be excluded if they cannot walk a minimum of $10 \mathrm{~m}$ or have other additional impairments that (severely) influence gait. Participants will receive 9 gait-training sessions over a 3week period and will be randomly allocated to an implicit or explicit group. Therapists are aware of the intervention they provide, and the assessors are blind to the intervention participants receive. Outcome will be assessed at baseline (T0), directly after the intervention (T1), and after 1 month (T2). The primary outcome parameter is walking velocity. Walking performance will be assessed with the 10-meter walking test, Dynamic Gait Index, and while performing a secondary task (dual task). Self-reported measures are the Movement Specific Reinvestment Scale, verbal protocol, Stroke and Aphasia Quality of Life Scale, and the Global Perceived Effect scale. A process evaluation will take place to identify how the therapy was perceived and identify factors that may have influenced the effectiveness of the intervention. Repeated measures analyses will be conducted to determine significant and clinical relevant differences between groups and over time.

Results: Data collection is currently ongoing and results are expected in 2019.

Conclusions: The relevance of the study as well as the advantages and disadvantages of several aspects of the chosen design are discussed, for example, the personalized approach and choice of measurements.

Trial Registration: Netherlands Trial Register NTR6272;

http://www.trialregister.nl/trialreg/admin/rctview.asp?TC=6272 (Archived by WebCite http://www.webcitation.org/6ytA937m5)

Registered Report Identifier: RR1-10.2196/9595

46 | Protocol of the Randomised Controlled Trial 


\section{BACKGROUND AND RATIONALE}

For most people, walking is a motor skill that generally takes place without too much effort. However, for people who have suffered a stroke, walking is often suddenly impaired, which can lead to major consequences in daily life functioning. People may experience impaired walking patterns with lower walking speeds, which has been associated with lower levels of functional ambulation.93 Gait training is one of the main components of physiotherapy within stroke rehabilitation because it is an important criterion for discharge and requirement for functioning at home.93,94 Evidence suggests that even in later (chronic) stages after stroke, people are still able to improve motor performance.95 Many different techniques and therapies can be used to improve walking performance 96; the challenge for physiotherapists is to choose and deliver gait training in the most efficient and effective manner. Moreover, preferably, obtained improvements in performance are durable over a longer period and resilient under different circumstances and in dual-task situations, for example, walking and talking. Despite the availability of new training approaches such as the use of robotics, 97 virtual reality, for example through exergames98,99 or body weight support training, 100 overground walking 101 still seems one of the most applied gait-training approaches in clinical practice. The current literature and clinical guidelines encourage the use of context- and task-specific treatment approaches, an example of which is overground walking. 96,102

To apply overground gait training, physiotherapists are encouraged to use general motor learning principles.102 Within the context of motor learning, a broad distinction between implicit and explicit motor learning has been described.4,8 Explicit motor learning can be defined as learning generated by verbal knowledge of movement performance; it involves cognitive stages within the learning process and is dependent on working memory involvement.27 The definition indicates that the learner is aware of all the underlying facts and rules of the to-be-learned motor skill during the process of learning. In practice, verbal explicit instructions are frequently used, and often these instructions encourage patients to be aware of their own body movements.6 For example, in gait training, therapists tell patients to think about their performance, for example, "Move your hips to the left and straighten your knee before stepping".6

In contrast to explicit learning, implicit motor learning progresses with no or minimal increase in the verbal knowledge of movement performance (eg, facts and rules) and without awareness.27 Learning is suggested to take place more automatically and in a less conscious manner. The learner is aware of the process of learning but cannot recall the underlying facts and rules of the motor skill. Gait training could be facilitated more implicitly, for example, when a physiotherapist would gradually constrain or change the environment, for example, when letting the person walk over different surfaces. 
In this situation, verbal instructions are not needed, but the environment facilitates the motor skill (walking). An observational study demonstrated that often multiple learning strategies are being used within one training session.92 These different learning strategies may represent an implicit-explicit continuum on which some promote more implicit and others more explicit forms of learning.4 Looking at the current practice, therapists seem to have a preference for learning approaches that are related to explicit learning in which often high numbers of verbal explicit instructions are used.6

Various advantages of implicit motor learning over explicit motor learning have been reported in literature. 8 Studies have demonstrated that individuals who learned motor skills implicitly perform the motor skill better under pressure, perform better in dual-task situations, and perform better over time compared with their explicit counterparts.8,9,12 Although these studies have primarily been conducted within the healthy population, implicit learning may also be advantageous for the patient population.50,91 For example, implicit motor learning may be of extra benefit to those with cognitive deficits.103 Reduced cognitive function is frequently seen in people after stroke63 and it often hampers the process of motor learning. The degree by which these cognitive functions are being evoked can be influenced by the choice of learning approaches.103 An interesting feature of implicit motor learning is the assumption that it is less reliant on working memory resources, that is, it involves less cognitive functions, compared with explicit motor learning.50 It is, therefore, intriguing to explore the effects of implicit motor learning within the stroke population.

Although from a theoretical perspective, the features of implicit motor learning have been described, its practical application in clinical practice seems more complex. Various learning strategies, for example, dual task, or errorless learning have been shown to promote implicit motor learning.12,25 One learning approach that may also be placed more on the implicit side of the implicit-explicit continuum is analogy learning. In analogy learning, the learner is provided with one single analogy or metaphor that strives to combine all the relevant rules of the to-be-learned motor skill. Early studies on analogy learning took place within a sporting context, for example, to learn specific skill techniques in table tennis or basketball.10,11 A good example in this regard was presented by Lam and colleagues.10 They used the analogy instruction "Shoot as if you are trying to put cookies into a cookie jar on a high shelf' to teach basketball players to impart backspin on the basketball. At present, there seems an increased interest of its application within different contexts. Analogy studies have been performed with older people 104 in the context of speech therapy22 and within neurological populations.24,68 For example, the analogy "imagine as if you are walking over a frozen lake" has been used in gait rehabilitation to facilitate lifting and placing the foot while walking.24 With regard to gait rehabilitation, small pilot studies have reported that analogies can be used in a feasible manner to facilitate walking performance.24,68 It has been reported that the analogy should 
lead to the desired biomechanical movement and that preferably the analogy should contain a meaningful component to the participant.68,85 In addition to feasibility, trends toward improved walking performance have been observed following analogy interventions, which demonstrates the potential of analogy learning in clinical practice.24,68 However, to further establish the effectiveness of analogy learning in clinical gait rehabilitation, larger sample sizes and research designs using a control condition are required.

This study describes the design for a randomized controlled study to assess the effects of implicit motor learning compared with explicit motor learning on walking speed in people suffering from stroke. The concept of analogy learning is used to structure the gait-training sessions within the implicit condition, whereas explicit motor learning is promoted by using extensive verbal instructions and feedback. A process evaluation is embedded to investigate feasibility and fidelity of the applied interventions.

\section{Research Question}

The following research question was established to examine the effects of implicit and explicit motor learning in gait rehabilitation of people after stroke: Is a 3-week implicit motor learning walking intervention (analogies) more effective compared with a 3-week explicit motor learning walking intervention (verbal detailed instructions) delivered at home with regard to walking speed in people suffering from stroke?

\section{METHODS}

\section{Study Design}

The study adopts a randomized, controlled, single-blinded study design in people suffering from stroke in the chronic stage of recovery. The study has been approved by the local ethics committee METC-Z in Heerlen, the Netherlands (NL number: NL.60338.096.16, Ethics nr: 17-T-06). After baseline measures, eligible participants will be randomized to the implicit or explicit condition (T0). Outcome assessments will take place directly after the intervention (T1) and again one month later (T2).

\section{Involvement of Client Representatives}

Throughout the design and planning of the study, 3 patient representatives were involved in every step of the decision-making process regarding the design and execution of the study. In several consensus meetings, they represented the patient's perspective, particularly with regard to the feasibility aspects of the study design. They were also involved in customizing participant information letters and promotion material for the study. 


\section{Population}

The study population consists of people who had a stroke and who are living at home. People will be recruited via local private practices, rehabilitation institutes, and a local health-related newspaper. Participants will be included if they had a stroke and want to improve their gait performance. To minimize the chance that improvements occur as a result of spontaneous recovery, only participants who are in the chronic stage of recovery (>6 months after stroke) will be included in the study. To prevent a ceiling effect, people with a low self-selected walking speed $(<1.0 \mathrm{~m} / \mathrm{s})$ will be invited to participate. Finally, all people should be able to communicate in Dutch and complete a 3-stage command. People are excluded if they are unable to walk a minimum distance of $10 \mathrm{~m}$; have a functional ambulation category score <3; have additional impairments not related to stroke, which influence their gait pattern, for example, severe osteoarthritis or amputation of the lower limb; have additional neurological impairments, for example, Parkinson disease that (severely) influence their walking performance.

\section{Sample Size}

The sample size calculation is based on a randomized controlled trial with equal group sizes and "walking speed" (10-meter walking test, 10MWT) as a primary outcome measure. 105 The power is set at beta $=.80$, the significance level at alpha $=.05$, and a standard deviation of $0.23 \mathrm{~m} / \mathrm{s} .106$ To demonstrate a significant change in walking speed, the minimal clinically important difference (MCID) is used and set at a minimum change of $0.16 \mathrm{~m} / \mathrm{s} .57,107$ The calculations resulted in a minimum group size of 33 participants per group. Taking into account that $20 \%$ (7/33) of participants may be lost during (dropout) and after the intervention (loss to follow-up), this study aims to recruit 40 participants per group.

\section{Randomization, Blinding, and Treatment Allocation}

\section{Randomization Procedure}

The allocation of participants to the experimental or the control condition will occur based on a computerized randomization program. Block randomization was calculated in block sizes of fours and sixes. The randomization procedure and the randomization scheme will only be available to an independent researcher who will not be involved in the delivery of the interventions or the performance of the measurements.

\section{Blinding}

The trained assessors are blind for treatment allocation. The therapists are aware of the treatment condition as they provide the explicit or implicit motor learning condition. The participants will probably also be aware of the treatment they receive; however, they will not be specifically told. The participants will be asked at each assessment not to reveal the details of treatment they received to the blinded assessor. 


\section{Training of Therapists and Treatment of Participants}

An intervention guideline is developed that outlines how the treatments (implicit and explicit conditions) should be delivered. The main aim of the intervention is to improve the quality of walking performance in people after stroke. The basic principle of the intervention guideline is based on the definitions of implicit and explicit motor learning by Kleynen et al.4 For the purpose of this study, we strive to create the largest contrast between the conditions as possible. The main characteristics and differences in instructions, and feedback of the practice between interventions are described in Figure 1. Both conditions will always be applied to an extent that is feasible for the participant, and training will therefore always be tailored to the participant's abilities within the given boundaries of the condition. Similar situations will be adopted in both conditions with regard to the "organization" of the training, for example, use variation in the (analogy) instructions and practice of the motor skill.

The chosen intervention period was based on a preliminary study of Kleynen et al 24 that demonstrated that 3 weeks was a feasible period to develop and practice analogies with people after stroke. All participants will receive 9 training sessions in a 3 -week intervention period, that is, 3 training sessions per week (Figure 2). Each training session takes place at the home of the participants and lasts for $30 \mathrm{~min}$. Participants were asked to use the instructions in daily life (unguided therapy) and after the 3-week intervention period. To standardize the training content as much as possible, the therapists involved in the study will be trained before the start of the study. During 5 standardization training sessions, the intervention guideline will be discussed, explicated, and the therapists will be trained with the help of example cases. During the trial, therapists will attend 3 evaluation sessions to discuss the progress of the study and possible cases or difficulties they may experience during the intervention.

\section{Implicit Condition}

Within the implicit condition, the concept of analogy learning is the leading approach as it has shown to adopt characteristics of implicit motor learning $11,24,68$ and provides therapists with a practical and feasible guideline to organize gait training. To choose and develop appropriate analogies, the same procedure as used by Kleynen et al 24 is followed. A booklet with examples of possible analogies will be available for the therapists and patients as a source of inspiration. Preferably, analogies are developed based on the participants' experiences and background to promote the personalized and meaningful aspects of analogy learning.24 All analogy instructions used in the trial will be documented. In addition to analogy learning, the therapists are allowed to use elements based on the characteristics of implicit motor learning as reported under the implicit column in Figure 1 . 


\section{Explicit Condition}

Within the explicit condition, gait training is organized by creating a learning environment that is (more) explicit in nature. Practice will be organized based on the characteristics of explicit motor learning as reported under the explicit condition in Figure 1. The condition is similar to the first (cognitive) stage of motor learning according to Fitts and Posners that is characterized by the use of many explicit instructions, explaining precisely how motor skills should be performed. This stage is verbal and cognitive of nature. Contrary to the implicit condition, the explicit condition strives to maximize the number of explicit verbal instructions. The explicit instructions that have been used in the trial will be documented by the therapist's in treatment logs.

\begin{tabular}{|c|c|c|}
\hline & More Implicit & More Explicit \\
\hline Elements & Implicit condition & Explicit condition \\
\hline General rules & $\begin{array}{l}\text { - Minimal use of verbal instructions. } \\
\text { - For the participant, learning should } \\
\text { progress without being aware of the } \\
\text { underlying gait parameters that the } \\
\text { therapist aims to improve. }\end{array}$ & $\begin{array}{l}\text { - Many verbalinstructions should be } \\
\text { used, however, only to an extent that } \\
\text { is feasible for the participant. } \\
\text { - For the participant, leaming should } \\
\text { progress in a conscious manner. } \\
\text { The participant should be very } \\
\text { aware of the underlying gait } \\
\text { parameters that the the rapist aims to } \\
\text { improve. }\end{array}$ \\
\hline \multirow[t]{2}{*}{ Instructions } & $\begin{array}{l}\text { The instruc tions should only contain } \\
\text { information about the analogy. The analogy } \\
\text { can be based on: } \\
\text { - Past experiences and/ormeaningful } \\
\text { situations. It should contain an image. }\end{array}$ & $\begin{array}{l}\text { Instructions should contain: } \\
\text { - Detailed instructions with reference } \\
\text { to joints, angles, and precise placing } \\
\text { of extremities/limbs. } \\
\text { - Biomechanical information. } \\
\text { - Keep intemally focussed (directed } \\
\text { on body movements). } \\
\text { - Create a conscious walking } \\
\text { environment. } \\
\text { - Frequently repeat verbal } \\
\text { instructions. }\end{array}$ \\
\hline & $\begin{array}{l}\text { - No information about joints, angles, } \\
\text { position of limbs, etc. } \\
\text { - No details about spatiotemporal gait } \\
\text { parameters orbiomec hanics of gait. }\end{array}$ & $\begin{array}{l}\text { - No information about how the } \\
\text { movement feels, no use of images, } \\
\text { no reference to familiar situations, } \\
\text { no imagery. }\end{array}$ \\
\hline Feedback & $\begin{array}{l}\text { - Knowledge of results (state goal of } \\
\text { movement). } \\
\text { - Link the feedback to the analogy e.g. } \\
\text { situations, images, or emotions. } \\
\text { - Use positive feedback. }\end{array}$ & $\begin{array}{l}\text { - Knowledge of performance } \\
\text { (kinematic feedback; information } \\
\text { about execution of movement). } \\
\text { - Link the instructions to e.g. feedback } \\
\text { directed at the joints, angles, or } \\
\text { placing of placing of } \\
\text { extremities/limbs. } \\
\text { - Use positive feedback. }\end{array}$ \\
\hline Examples & $\begin{array}{l}\text { - Walk as if you are walking through a } \\
\text { deep layer of snow. } \\
\text { - Walk as if you follow the footprints in the } \\
\text { sand. } \\
\text { - Try to cross a small bridge. }\end{array}$ & $\begin{array}{l}\text { - Place yourheel right. } \\
\text { - } \text { Lift your feet. } \\
\text { - } \text { Transferyour weight on your } \\
\text { right/left leg. } \\
\text { - Push off with your foot. } \\
\text { - Bend yourknees. } \\
\text { - } \text { Walk up-right. } \\
\text { - Lift yourwalking frame. }\end{array}$ \\
\hline
\end{tabular}

Figure 1. Characteristics of the interventions. 


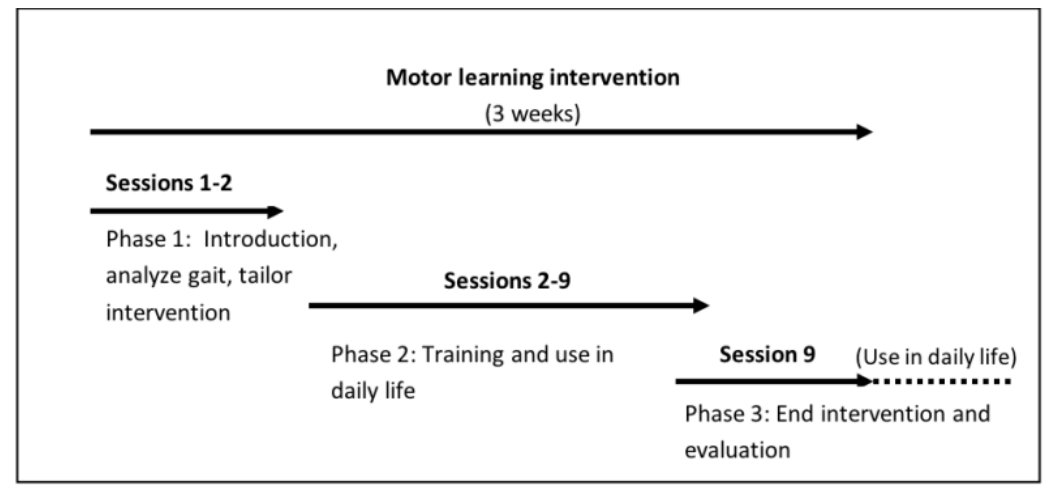

Figure 2. Overview of the gait training sessions.

\section{Measurements}

All measures will be assessed by independent, blinded, and trained assessors at 3 assessment points (T0, T1, and T2; Table 1) and will take place at the participant's home. The primary outcome parameter is walking speed measured in meters per second using 10MWT. First, demographics are described, and then, the primary and secondary outcome measures are reported.

Table 1. Overview of measurements used in this study.

\begin{tabular}{|c|c|c|}
\hline Data & Time & ICF a level \\
\hline \multicolumn{3}{|l|}{ Demographics } \\
\hline $\begin{array}{l}\text { Age, gender, time poststroke, affected or } \\
\text { nonaffected side, walking aids, educational level }\end{array}$ & T0 & Personal factors \\
\hline Montreal Cognitive Assessment & T0 & Impairment level \\
\hline Berg Balance Scale & T0 & Activity level \\
\hline Rivermead Mobility Index & T0 & Activity level \\
\hline Fugl-Meyer Assessment & T0 & $\begin{array}{l}\text { Body functions and } \\
\text { structure }\end{array}$ \\
\hline \multicolumn{3}{|l|}{ Walking performance } \\
\hline 10-Meter Walking Test & $\mathrm{T} 0, \mathrm{~T} 1, \mathrm{~T} 2$ & Activity level \\
\hline Dynamic Gait Index & $\mathrm{T} 0, \mathrm{~T} 1, \mathrm{~T} 2$ & Activity level \\
\hline Dual task & $\mathrm{T} 0, \mathrm{~T} 1, \mathrm{~T} 2$ & Activity level \\
\hline \multicolumn{3}{|l|}{ General outcome measures } \\
\hline Movement Specific Reinvestment Scale & $\mathrm{T} 0, \mathrm{~T} 1, \mathrm{~T} 2$ & Personal factors \\
\hline Verbal protocol & $\mathrm{T} 0, \mathrm{~T} 1, \mathrm{~T} 2$ & N/A \\
\hline Stroke Specific Quality of Life Scale & T0, T2 & Participation level \\
\hline Global Perceived Effect scale & $\mathrm{T} 2$ & N/A \\
\hline
\end{tabular}

aICF: International Classification of Functioning, Disability, and Health.

bN/A: not applicable. The ICF level is not applicable for the Verbal Protocol and Global Perceived Effect Scale as these measures do not examine health or disability but evaluate the intervention. 


\section{Demographics}

At baseline, the following demographic and prognostic information is collected (T0): age, gender, time post stroke, affected side, walking aids, educational level, cognitive level (Montreal Cognitive Assessment, MoCA)78, static balance and fall risk (Berg Balance Scale)108, measures of mobility and disability (Rivermead Mobility Index)109, and ability to make movements outside the synergetic patterns (Fugl-Meyer assessment of the lower limb).110 To assess the propensity for conscious motor processing, the Dutch version of Movement Specific Reinvestment Scale (MSRS) is used. 111,112

\section{Walking Performance Measures}

Walking performance is measured using the 10MWT71 and the Dynamic Gait Index (DGI).113 To assess the robustness of the obtained performance, walking will also be assessed over a longer period (1-month follow-up) and under secondary task loading.

\section{0-Meter Walking Test}

Gain in walking speed has been associated with a transition to a higher class of ambulation, resulting in a better function and quality of life.71,114 Next to statistical significance, the MCID will be used to assess clinical relevant differences. The MCID for walking speed in people after stroke has been established at the minimal difference of $0.16 \mathrm{~m} / \mathrm{s} .107$ Exceeding this threshold indicates that the participants obtained a clinically meaningful improvement.

\section{Dynamic Gait Index}

The DGI is a physical performance test that assesses the gait, balance, and fall risk and has shown to have a good reliability and validity in people after stroke.115,116 Eight different tasks related to the balance and gait, for example, walking, turning, and stepping over objects are assessed.117 The performance will be scored according to the modified DGI as proposed by Shumway and Cook as the extended scoring system has shown to possess good psychometric properties.113,118

\section{Dual Task}

In this study, people will be asked to complete a tone-counting task similar to that proposed by Wilson et al.119 In this task, people will be exposed to 4 different sounds (buzzer, ping, tone and bell ring) in a randomized order over a 30-s time period. They will be asked to only count a specific target sound (eg, bell ring) and ignore the other 3 distracting sounds. The task will be performed twice, once as a single task and once while walking concurrently (dual task). The actual number of tones will be compared with the estimate number of tones by the participants. Error scores (actual minus estimate) will be calculated as a measure for the dual-task performance. 


\section{Self-Reported Measures}

\section{Movement-Specific Reinvestment Scale Adapted for Gait}

The MSRS is a questionnaire that measures a person's inclination for conscious control. People after stroke have shown to have greater propensity to conscious processing compared with the age-matched, nondisabled population.120,121 In this study, an adapted version of the MSRS specific to gait is used. Adapted versions of the MSRS have been used before, for example, for putting movements in golf.122,123 The MSRS contains one factor related to conscious control (conscious motor processing) and one related to selfconsciousness about movement (movement self-consciousness). Each factor of the MSRS comprises 5 statements, such as "I try to think about my movements when walking" (conscious motor processing) and "I am concerned about what people think about me when I am walking" (movement self-consciousness). The statements will be assessed with binary response (yes/no).111 The MSRS will be measured at baseline (T0) to describe the population and over time (T0, T1, and T2) to assess how much gait-related conscious processing takes place.

\section{Verbal Protocol}

To assess the amount of explicit knowledge, a verbal protocol questionnaire as used in Orrel et al will be administered after the 3-week intervention.25 Explicit knowledge is assessed by examining the number of explicit rules that the participant uses during walking. Participants will be asked to report any "rules, methods, or techniques" that they have thought about or used and that have improved or impaired their walking performance. A rule is defined as any statement containing at least one of the following aspects: a movement or position of one limb, a movement or position of one joint, a velocity of a limb movement, an angle or direction of a joint or the spine, or the placement of the walking aid. Each statement containing a single limb, joint, or other body part will be counted as 1 rule. If a statement contains 2 (or more) different limbs, joint and body parts, or different directions or angles, they are counted separately (eg, "I tried to lift my foot and put it more forward."). Statements are excluded if they are irrelevant to walking performance or do not refer to technical aspects about walking (eg, "More concentration needed."). The answer to the verbal protocol will be screened by 2 independent researchers who will be blind to the experimental condition. Their agreement will be investigated using a correlation coefficient (or ICC).

\section{Stroke and Aphasia Quality of Life Scale}

The Stroke and Aphasia Quality of Life Scale (SAQOL-39) is assessed at baseline and after the intervention to measure the health-related quality of life.124 The questionnaire contains 39 items and is developed for people after stroke and is feasible to use for people with aphasia. The SAQOL-39 is a short version of the original SAQOL (53 items) and has 
shown to be an acceptable, reliable, and valid measure of the health-related quality of life. 124

\section{Global Perceived Effect}

The Global Perceived Effect scale is a reliable method to assess the participant's satisfaction and will be used to evaluate the participant's perception of the intervention.125 The Global Perceived Effect scale will involve the following question: To which extent did your walking ability change over the last three weeks? The question will be scored on a 7-point Likert scale from "completely improved" to "completely deteriorated."

\section{Process Evaluation}

To gain an insight into the process-related factors that may have influenced the effectiveness of the 3-week analogy learning walking intervention, a process evaluation will take place along the study.126 Data will be collected to (1) asses to what extent the therapists delivered the interventions as intended (fidelity), (2) explore the therapists' opinions and experiences about the interventions with regard to the feasibility and possible effects, and (3) explore the patients' opinions and experiences about the interventions with regard to the feasibility. Table 2 represents the data collection methods used to assess the different aspects of the process evaluation. For both groups, the provided instructions will be documented and evaluated in the therapists' logs. In addition, short questionnaires will be used to administer the therapists' and participants' opinions about the gait training after the completion of the 3-week intervention. To explore the extent to which the interventions were implicit or explicit in nature, the verbal protocol will be assessed. Evidence indicates that implicit learning is typically characterized by the less accumulation of explicit rules compared with explicit learning.11,127

To monitor the integrity of the intervention, self-reported (subjective) and audio-recorded (objective) data will be evaluated. First, all therapists are required to self-report any deviations from the treatment protocol or other incidents during the session in a log after every session. All instructions used during therapy sessions will be recorded in the therapists' and patients' logs. Furthermore, patients can use the log to write down any possible events that might have occurred during unguided therapy. The patient log is, therefore, only used as a reminder for unguided therapy and as a communication tool between therapists and participants. Second, 10 gait trainings will be randomly selected (5 implicit and 5 explicit interventions) and audio recorded. Both self-reported and audiorecorded data will be screened to evaluate whether the intervention was delivered according to the protocol. 
Table 2. Measures for the process evaluation. A checkmark indicates with which measure the question is examined.

\begin{tabular}{|c|c|c|c|c|}
\hline \multirow[t]{2}{*}{ Questions } & \multicolumn{4}{|c|}{ Measures } \\
\hline & $\begin{array}{c}\text { Therapist } \\
\text { Log }\end{array}$ & $\begin{array}{c}\text { Audio } \\
\text { recordings }\end{array}$ & $\begin{array}{c}\text { Therapist } \\
\text { questionnaire }\end{array}$ & $\begin{array}{c}\text { Patient } \\
\text { questionnaire }\end{array}$ \\
\hline $\begin{array}{l}\text { To what extent did the therapists } \\
\text { deliver the interventions as intended } \\
\text { (fidelity)? }\end{array}$ & $\checkmark$ & $\checkmark$ & $\checkmark$ & \\
\hline $\begin{array}{l}\text { How did the therapist's experience } \\
\text { delivering the interventions with } \\
\text { regard to the feasibility and possible } \\
\text { effects? }\end{array}$ & & & $\checkmark$ & \\
\hline $\begin{array}{l}\text { How did the patients' experience the } \\
\text { intervention with regard to the } \\
\text { feasibility? }\end{array}$ & & & & $\checkmark$ \\
\hline
\end{tabular}

\section{Data Analyses}

Baseline scores of demographic and prognostic data and primary and secondary outcome measures will be used to compare the 2 groups. Only data of the participants who attended minimum of 7 or more of the therapy sessions will be considered as adherent and processed in the statistical analysis. Statistical analysis will be conducted to determine significant differences between groups and over time (baseline and postintervention performance). A repeated measure analyses will be used to compare the 2 groups (implicit and explicit) at 3 different time points (before, after, and after 1-month follow-up). Post hoc tests with correction for multiple testing will be used to explore effects over time and between groups. Subgroup analysis will be performed on cognition (MoCA score <21). 78 An alpha level of .05 will be set for all tests. The primary outcome measure, walking speed, will also be described with reference to clinically relevant differences between groups (MCID: $0.16 \mathrm{~m} / \mathrm{s}$ ).107 All datasets used or analyzed during this study are available from the corresponding author on a reasonable request.

Data will be analyzed according to an intention-to-treat and per-protocol principle. In the intention-to-treat, data of the participants are analyzed according to their original treatment allocation. If self-reported (subjective) and audio-recorded (objective) data reveal that cases are not delivered as intended, then the analyses will be performed using the perprotocol principle. Within this study, protocol deviations are defined as "deviations from the protocol that occur in two or more sessions." If protocol deviations were observed, then data from this person were not included in the per-protocol analysis. Data in the process evaluation related to the therapists' and patients' opinions and experiences toward the feasibility of the intervention and perceived benefits will be analyzed by means of descriptive statistics. Free comments and clarifying examples may be quoted and used to describe personal experiences of the therapists or participants. 


\section{RESULTS}

The entire project was funded in September 2015. Patient enrolment began in March 2017 and is expected to continue until July 2018. Following completion of data collection, data cleaning and analyses will take place. The first study results are expected to be submitted for publication in 2019.

\section{DISCUSSION}

In this paper, we described the methodology of a randomized controlled single-blinded study that evaluates the potential effects of implicit motor learning compared with explicit motor learning in the gait rehabilitation of people suffering from stroke. The relevance of the study and the advantages and disadvantages of several aspects of the chosen design are discussed.

\section{From Laboratory Setting to Clinical Practice}

Although motor learning research is growing exponentially, most published studies have been performed in laboratory settings. However, it is important to understand the application of motor learning within clinically relevant environments and the influence of interventions on the completion of everyday functional tasks.91,127 A recent systematic review on implicit motor learning in people after stroke pointed out that studies performed within clinical settings are limited.91 Of the 20 included studies, only 1 study involved a clinically relevant task.25 To improve the generalizability of research findings toward clinical practice and to the broader population, various choices on different aspects in the research design had to be made. First, with regard to the task and environment, this study involves gait training that takes place at the homes of the participants. Gait is a functional daily life motor skill, and it is advised to organize practice in a context-specific environment.96,128 Therefore, compared with the current state of evidence, this study adds insights into the effects of motor learning in a clinically relevant environment and for a clinically relevant task.

Second, in this study, the motor learning conditions are tailored to the individual participants. Most implicit motor learning studies use strict research protocols in which each participant usually receives the exact same instructions (eg, the whole experimental group receives the same right-angled triangle analogy to learn topspin forehand in table tennis), whereas the explicit learning is promoted by using the same set of verbal instructions.11 A one-size-fits-all approach may not be ideal for the clinical population as they generally demonstrate a large variety in degree and types of impairments. The use of personalized analogies allows the physiotherapists to respond to the individual walking impairments and emphasize on the meaningful component of analogy learning. With a personalized approach (more comparable with the real-life practice), the instructions may 
be less standardized. Therefore, steps were undertaken to ensure the quality of the interventions. Before the study, all therapists were familiarized and trained with the implicit and explicit conditions. Measures (therapists' logs and audio recordings of the interventions) were selected to evaluate whether the trainings were delivered as intended, and data will be analyzed as per the intention-to-treat and per-protocol (see the Data Analyses section).

\section{Methodological Aspects}

Within this study, extra attention was given to the following 3 methodological aspects in designing the intervention: contrast, content of the interventions, and target population. First of all, it is important to address that in many rehabilitation studies, the contrast between the intervention and control group (generally usual care only) turns out to be too modest, which therefore results in neutral study results.129 The underlying reason may be that rehabilitation interventions are often too complex to control for influences of other interventions and that the control intervention group (therapy as usual) is often poorly described. We tried to overcome these problems by including participants in the chronic phase of recovery, who do not receive additional interventions directed at the improvement of gait. Furthermore, we strive to ensure the contrast of the interventions by using a guideline, written protocol. In this protocol, the delivery of both conditions is clearly defined. It transparently describes how the 2 interventions differentiate from each other. To ensure the integrity of the intervention, the logs and audio-recorded therapy sessions will be conducted and evaluated.

Another decision we would like to address is the choice for the interventions. In clinical practice, often mixtures of implicit and explicit forms of learning are used or therapists switch between different forms of learning.92,130 However, it remains unclear whether mixing implicit and explicit learning or switching between the forms is effective and/or necessary. The current design is necessary to evaluate the effects of implicit versus explicit learning in clinical practice, and so we believe that it is (ethically) legitimate to compare 2 distinct interventions and not use "therapy as usual" as a control intervention. The interventions in this study will be delivered in a personalized manner regarding overall components of the interventions, for example, the sort of gait impairments, amount of repetitions, and use of personalized analogies. However, the content of the interventions applied is clearly outlined in the guidelines, and therapists are required to strictly follow these.

Furthermore, within the target population, people experiencing cognitive impairments or communicative restrictions are often excluded. In this study, we strive to include a sample that reflects the broad range of impairments reported after stroke. In earlier studies, it has been shown that motor learning interventions might be effective, also for people with cognitive and/or communicative impairments.24,68 


\section{Implicit Motor Learning}

Scientific evidence describes that implicit learning is typically characterized by robust dual-task performance, durable performance over time, and less accumulation of explicit rules.11,127 The dual-task measure and the 1-month follow-up session were specifically chosen with respect to these implicit characteristics. First, walking while concurrently carrying out a secondary (tone counting) task119 places high information-processing demands on the learners. In contrast to explicit learners, implicit learners showed that performance was not disrupted in dual-task conditions, which indicates that they may be able to free up attentional resources to perform the secondary task.11 We therefore predict that the implicit condition will remain stable under secondary task burden. For this reason, implicit learning may be particularly beneficial for those who experience cognitive impairments that are commonly seen in people after stroke.

Then, a recent study by Tse et al104 found performance improvements after a 2-day separation; however, they recommended to include a longer separation to test skill consolidation. This study will therefore evaluate performance after the 1-month follow-up period. It is hypothesized that performance improvements in the implicit group will remain robust over a longer period (1-month follow-up). To make statements about long-term effects within rehabilitation, it may be desirable for future studies to include even longer follow-up periods (>3 months). Finally, a verbal protocol will be assessed because it is hypothesized that participants within the implicit learning group will report fewer explicit rules than the control group, which would be in line with the findings of earlier studies.10,11 Even though the starting point of the explicit intervention is to use many verbal explicit instructions and provide more details on the motor performance, the exact number of explicit rules will be tailored to the ability of the patient to process these rules. Therefore, in practice, some patients might receive higher numbers of explicit rules than others. Still, we hypothesize that the explicit group will require more explicit rules compared with the implicit group.

\section{Conclusion and Implementation}

With the description of the study design, we hope to contribute to the discussion on how a tailored but standardized form of implicit motor learning could be applied in clinical practice. The relevance of the study and the advantages and disadvantages of several aspects of the chosen design are discussed (eg, personalized approach, sample selection). 


\section{Acknowledgments}

The authors would like to thank all (consortium) partners of the research project "Power of Implicit Motor Learning 2.0" for their participation in research meetings and thoughts along the planning and design of the study. The authors would like to thank the therapists and researchers Jos Halfens, Jos Kurvers, Kyra Theunissen, Monique Rothbauer, Inge de Lang, and Ritch te Kampen for their expertise and thoughts in designing the therapist's guidance and measurement protocols. The authors would also like to thank their client representatives Nathalie Sieben, Else de Bont, and Anja Minheere for their valuable perspectives and thoughts about the trial along the set-up of the study. The authors would like to thank Kirsty Brock for helping improve the language of the manuscript. This study is financially supported by Stichting Alliantie Innovatie (Innovation Alliance Foundation), RAAK-international (registration number: 2011-3-33int).

\section{Authors' Contributions}

LJ, MK, AB, and SB conceived and designed the study. LJ, MK, and SB wrote the first version of the manuscript. $\mathrm{LJ}, \mathrm{MK}, \mathrm{KM}, \mathrm{AB}$, and $\mathrm{SB}$ critically revised the manuscript for the relevant intellectual content. All authors read and approved the final version for submission.

\section{Conflicts of Interest}

None declared. 
$62 \mid$ Protocol of the Randomised Controlled Trial 


\section{CHAPTER 5}

\section{Implicit and explicit motor learning interventions have similar effects on walking speed in people after stroke: a randomized controlled single blind trial.}

Li-Juan Jie, Melanie Kleynen, Kenneth Meijer, Anna JHM Beurskens, Susy M Braun

Submitted, available as pre-print form as:

Jie, L. J., Kleynen, M., Beurskens, A., Meijer, K., \& Braun, S. (2019). Implicit and explicit motor learning interventions have similar effects on walking speed in people after stroke: a randomized controlled single blind trial. medRxiv. doi: 0.1101/19008797.

(C) The Authors 2019. This work is licensed under the Creative Commons Attribution 4.0 International License. http://creativecommons.org/licenses/by/4.0/ 


\begin{abstract}
Background: Therapists may use (more) implicit or (more) explicit motor learning approaches to facilitate motor skill learning of stroke patients. The use of implicit motor learning approaches has shown promising results in healthy populations.

Objective: To assess whether an implicit motor learning walking intervention is more effective compared to an explicit motor learning walking intervention delivered at home with regard to walking speed in people after stroke in the chronic phase of recovery.

Design: Randomized controlled single blind trial.
\end{abstract}

Setting: Home environment.

Patients: 79 people in the chronic phase after stroke $(66.4 \pm 11.0$ years; $70.1 \pm 64.3$ months after stroke; walking speed $0.7 \pm 0.3 \mathrm{~m} / \mathrm{s}$; Berg Balance Scale score $44.5 \pm 9.5$ ) were randomly assigned to an implicit $(n=38)$ or explicit $(n=41)$ group.

Intervention: Analogy learning was used as the implicit motor learning walking intervention, whereas the explicit motor learning walking intervention consisted of detailed verbal instructions. Both groups received nine training sessions, 30 minutes each, for a period of three weeks.

Measurements: The primary outcome was walking speed measured by the 10-Meter Walk Test. Outcome measures were assessed at baseline, immediate and 1-month post intervention.

Results: No statistically or clinically relevant differences between groups were obtained post intervention (between-group difference estimated $0.02 \mathrm{~m} / \mathrm{s}$ [95\% CI -0.04 to 0.08 ] and at follow-up (between-group difference estimated $-0.02 \mathrm{~m} / \mathrm{s}$ [95\% CI -0.09 to 0.05 ], $\mathrm{p}=0,563)$.

Limitations: The treatment effects may have been diluted by "noise" accompanied with research within real life settings, complex tasks and a representative sample.

Conclusions: Implicit motor learning was not superior to the explicit motor learning to improve walking speed in people after stroke in the chronic phase of recovery

$64 \mid$ Results of the Randomised Controlled Trial 


\section{INTRODUCTION}

One of the most practiced motor skills in stroke rehabilitation is walking.131 In general, therapists use (more) implicit or (more) explicit forms of learning to facilitate improvement of gait. Explicit motor learning can be referred to as a more conscious form of learning, that is characterized by the generation of verbal knowledge (i.e. facts and rules about movement performance) and involvement of cognitive resources.38 In contrast, implicit motor learning is assumed to take place without much knowledge of the underlying facts and rules of motor skills and has been described as "learning that progresses with no or minimal increase in the verbal knowledge of movement performance and without awareness'.27 Within current clinical practice therapists tend to structure therapy in a more explicit manner or switch between implicit and explicit learning approaches.6,92,130 However, this might not always be efficient. For people after stroke, who often experience cognitive impairments.28 it can be difficult to process large amounts of verbal explicit information. Implicit motor learning, on the other hand, strives to minimize the involvement of cognitive resources, especially working memory 65 and may therefore be more feasible for people after stroke who apart from physical constraints also suffer from cognitive impairments. Studies show that people after stroke are able to learn implicitly and that performance of an implicitly learned task might be more stable under dual-task condition and more durable over time.25 However, there is still a lack of studies comparing the effects of implicit motor learning post-stroke to explicit motor learning within clinically relevant tasks. In order to be clinically meaningful, implicit and explicit motor learning approaches need to be tailored to the individual needs of the patients and performed in the real-life situations.

One practical approach to induce implicit motor learning is through the use of analogies. In analogy learning, the learner is provided with one single metaphor (or analogy) that strives to encompass all underlying (explicit) knowledge that is necessary to complete the motor skill. For example, to facilitate step length a therapist could provide the analogy 'Walk as if you follow the footprints in the sand'.132 Although no technical (explicit) instructions are given, the analogy may facilitate for example a more symmetrical gait, the foot strike from heel to toe and foot-clearance. Studies in athletes have shown that analogy learning led to better and more stable performance under dual-task conditions.10,11 Within the neurological population first pilot studies reveal the feasibility of analogy learning and demonstrate its potential as both clinically relevant and statistically significant changes in walking performance could be obtained.24,68,132 In the current study, the effects of analogy learning were compared to detailed verbal instructions when training the clinically relevant task 'walking' in a real life setting (home environment).

To our knowledge, this is the first randomized controlled trial that examines the effects of implicit motor learning facilitated by analogies compared to explicit motor learning on a 
functional walking task in people after stroke. Contrary to earlier studies examining implicit motor learning using the same analogy for the entire group 10 the current study also tailored the interventions towards the individual needs, preferences and abilities of the patients. The research question was: Is a 3-week implicit motor learning walking intervention (analogies) more effective compared to a 3-week explicit motor learning walking intervention (verbal detailed instructions) delivered at home with regard to walking speed in people after stroke who are in the chronic phase of recovery? Walking speed was chosen due its integrated results on other gait parameters e.g. step length 133 and functional outcomes.134 It was hypothesized that implicit motor learning would result in greater improvements of walking speed post intervention (especially at 1-month post intervention).

\section{METHOD}

\section{Study design and participants}

The study adopts a randomized, controlled, single-blinded study design and was approved by the local ethics committee METC-Z in Heerlen, the Netherlands (approval number 17T-06, Netherlands Trial Register: NL6133). Full details of the study protocol have been published elsewhere.135 Recruitment of participants took place via community practices, rehabilitation institutes in the region and through a local health-related newspaper. Participants were included if they were $>6$ month after stroke, had a self-selected walking speed lower than $1.0 \mathrm{~m} / \mathrm{s}$, were able to communicate in Dutch and to complete a threestage command. Participants were excluded if they were unable to walk a minimum distance of 10 meter, could not ambulate on level surfaces without manual contact of another person (Functional Ambulation Scale (FAC) < 3), had additional impairments not related to stroke that significantly influenced their gait pattern (e.g. Parkinson's disease). All participants signed a written informed consent.

\section{Randomization and masking}

A randomization list was generated using a web-based randomization program and was only available to an independent researcher, not involved in the delivery of the interventions or measurements. Patients were randomly assigned (1:1) to either the implicit or explicit motor learning condition (block size of four). The assessors were blind to the treatment allocation. The therapists were aware of the treatment condition they provided. Patients were not told which condition they received and were asked to not reveal details about the treatment to the blinded assessors.

\section{Interventions}

In total nine training sessions were provided over a three-week long intervention period. Each session lasted 30 minutes. Within a case-study this duration and frequency of sessions were sufficient to result in clinically meaningful changes.24 An intervention 
guideline outlining how the implicit and explicit motor learning intervention should be delivered was developed for therapists in the trial. The guideline was developed with physiotherapists and client representatives and was based on the previous pilot studies and experiences.24,132,135 Prior to the trial, five standardization training sessions with the therapists took place to discus and explicate the intervention guideline with example cases. In both interventions, therapist examined the participants walking pattern and defined the underlying gait parameters which could potentially influence walking speed. More details about the interventions and main characteristics with regard to instructions and feedback are described in Figure 1.135

\section{The implicit intervention}

The main focus for the implicit intervention was creating a learning situation in which the learner was not (or minimally) aware of the underlying rules of the practiced motor skill. The concept of analogy learning formed the basis to guide the implicit intervention because 1) it has shown to adopt characteristics of implicit learning 11 and 2) it offers therapists a practical and feasible tool to apply therapy.24,68 The participants were provided with an analogy which aimed to improve the walking performance and was meaningful to them.

\section{The explicit intervention}

The main focus for the explicit intervention was creating a learning situation in which the learner is very aware of the learning process, e.g. in which he/she can precisely explicate the underlying facts and rules that are necessary to perform the motor skill. Therefore, the participant was provided with detailed explicit instructions on their gait performance e.g. "While walking pay attention your foot placement and motion. Place your left foot in front of your right foot. Make sure that you strike the ground with your heel first. Then roll through from heel to toe. Finally push off with your toe."

\section{Outcomes}

\section{Demographic information}

The following demographic information and clinical characteristics were collected: age, gender, time post stroke, affected side, use of walking aids, educational level, cognitive level (Montreal Cognitive Assessment, MoCA)78, static balance and fall risk (Berg Balance Scale)108, mobility disability (Rivermead Mobility Index)109, and ability to make movements outside the synergetic patterns (Fugl-Meyer assessment of the lower limb) 110 . To assess the propensity for conscious motor processing, the Dutch version of Movement Specific Reinvestment Scale (MSRS)111,112 was used. 


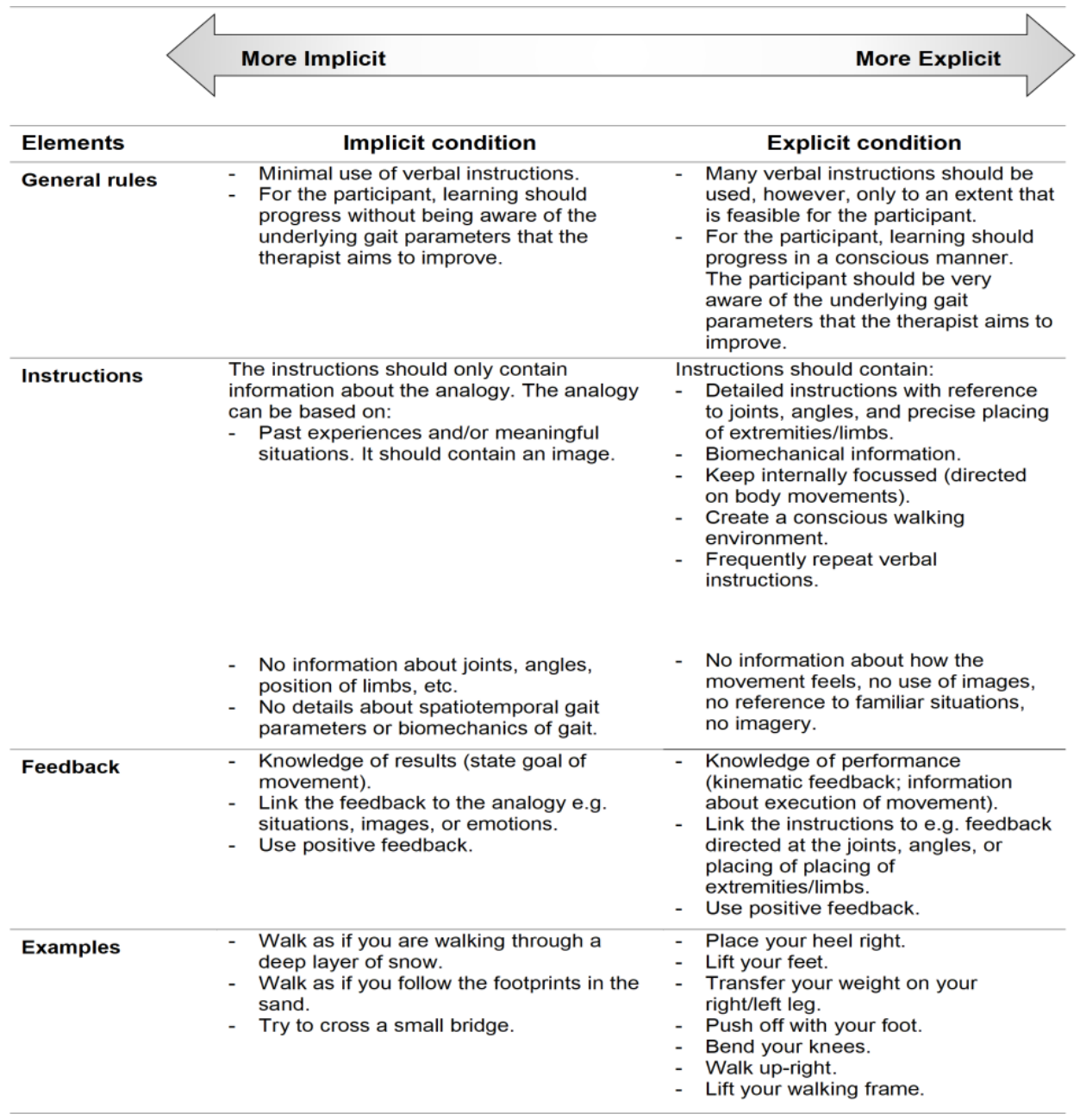

Figure 1. Characteristics of the interventions. Adapted from Jie et al.135

\section{Outcome assessment}

The primary outcome measure was walking speed which was calculated by measuring the walking time on a 10-Meter Walk Test (10MWT; 10m / time (s)).71 Secondary outcomes measures were the modified Dynamic Gait Index (DGI),113 motor and cognitive Dual Task (DT) performance,119 Movement Specific Reinvestment Scale (MSRS) adapted for gait,120,135 verbal protocol, Stroke and Aphasia Quality of Life Scale (SAQOL-39),124 Global Perceived Effect (GPE) scale 125 and verbal protocol. 
Assessment of the dual task

Motor task performance was measured through calculating the Dual Task Effects (DTE) according to the formula of Kelly et al.136 The cognitive task performance was measured via calculating the error scores as done before by Wilson et al.119 The error scores were not yet relative to single task. Therefore, the dual task error scores were subtracted from the single task error scores. Both the motor and cognitive task performances were expressed in percentages. Negative percentages indicate that performance deteriorated relative to single task, whereas positive scores indicate relative improvements of the dual task performance.

\section{Verbal Protocol}

To assess the amount of explicit knowledge, a verbal protocol questionnaire was be administered after the three-week intervention.25 Explicit knowledge is assessed by examining the number of explicit rules that the participant used during walking. More information of the definition of 'explicit rule' is described elsewhere.135 The answers of the verbal protocol were screened by two independent researchers who were blind to the experimental intervention.

\section{Sample size calculation and statistical analyses}

The sample size calculation resulted in a minimum group size of 33 participants per group. The power was set at beta 0.80 , the significance level at alpha 0.05 , and a standard error of $0.23 \mathrm{~m} / \mathrm{s} .106$ The minimal clinically important difference (MCID) of $0.16 \mathrm{~m} / \mathrm{s}$ for walking speed was set as the minimal change. 107 Considering $10 \%$ of participants may be lost during (drop-out) and another $10 \%$ after the intervention (loss-to-follow-up), this study aimed to recruit 40 participants per group. The statistical analyses were performed using IBM SPSS (version 24). Baseline characteristics of the two groups were reported using frequency distributions and descriptive statistics. For the intention-to-treat analyses data of all participants who received the intervention were analysed according to their original treatment allocation. Treatment effects on numerical data were assessed using a linear mixed model. The model represented group, time and group $\times$ time as fixed factors. For the repeated measures (balanced design) an unstructured covariance structure was used. The linear mixed model analyses uses all available data, corrects for baseline differences and accounts for dependency of data.

Statistical analyses of the primary outcome was also described in relation to clinically relevant differences between groups (MCID: $0.16 \mathrm{~m} / \mathrm{s}$ ). 107 In the per-protocol analyses data of subjects were excluded if they did not receive the intervention as intended i.e. when protocol deviations occurred in two or more (of the nine) sessions. Possible protocol deviations were self-reported (subjective) in therapists logs and randomly 10 gait training sessions were audio-recorded (objective) and evaluated to detect protocol deviations. Furthermore, people who did not meet the inclusion criteria or people who dropped out 
were excluded in the per-protocol analysis. Descriptive sub-group analysis was performed on cognition to explore whether cognitive abilities (MoCA $\leq 21)$ might influence the effect of the interventions. The verbal protocol was only assessed once and an independent t-test was used to compare results between the groups.

\section{RESULTS}

\section{Flow of participants through the trial}

The flowchart of the trial is presented in figure 2. Between 19 May 2017 and 19 September 2018, a total 81 people were assessed for eligibility and randomized. Two participants (3\%) did not start with the study. One participant withdrew due to diagnoses with additional impairments that severely influenced his gait. The other participant decided to stop due to personal reasons. All participants $(n=79)$ that started the intervention were included in the primary intention-to-treat analysis. Demographics and baseline characteristics are presented in table 1 . There were no apparent differences between the groups at baseline.

\section{Compliance with the trial}

Two participants (3\%) deviated from the protocol with regard to the provided instructions (>2 explicit instructions within the implicit intervention). Analysis revealed that in retrospect ten participants $(13 \%)$ did not meet the inclusion criteria of walking slower than $1 \mathrm{~m} / \mathrm{s}$ at baseline. In addition, three participants (4\%) wanted to improve overall fitness but had no specific goals related to gait and therefore discontinued with the intervention. Two participants (3\%) stopped due to other complaints not related to gait. Furthermore, the medical diagnoses of one participant (initially stroke; $1 \%$ ) was changed during the intervention. Due to pregnancy another participant dropped out of the intervention. All available data of these 19 participants $(24 \%)$ were included in the primary intention-totreat analysis but were excluded in the per-protocol analysis. 
Table 1. Baseline characteristics of participants.

\begin{tabular}{|c|c|c|}
\hline & $\begin{array}{l}\text { Implicit } \\
(n=38)\end{array}$ & $\begin{array}{l}\text { Explicit } \\
(n=41)\end{array}$ \\
\hline \multicolumn{3}{|l|}{ General Characteristics } \\
\hline Age $(y r)$, mean (SD) & $64.6(9.4)$ & $67.8(11.6)$ \\
\hline Gender, $\mathrm{n}$ males $(\%)$ & $24(63.2 \%)$ & $25(61.0 \%)$ \\
\hline Length $(\mathrm{cm})$, mean (SD) & $171.7(8.0)$ & $172.0(8.8)$ \\
\hline \multicolumn{3}{|l|}{ Educational level, n (\%) } \\
\hline Elementary education & $0(0.0 \%)$ & $1(2.4 \%)$ \\
\hline Secondary education & $20(52.6 \%)$ & $18(43.9 \%)$ \\
\hline Vocational training & $10(26.3 \%)$ & $11(26.8 \%)$ \\
\hline University & $8(21.1 \%)$ & $11(26.8 \%)$ \\
\hline \multicolumn{3}{|l|}{ Stroke Characteristics } \\
\hline Time post stroke (months), mean (SD) & $72.8(59.3)$ & $67.5(69.1)$ \\
\hline Side of stroke, $\mathrm{n}$ right $(\%)$ & $19(50.0 \%)$ & $16(39.0 \%$ \\
\hline \multicolumn{3}{|l|}{ Independent walking, $\mathrm{n}(\%)$} \\
\hline Walk unaided & $9(23.7 \%)$ & $9(22.0 \%)$ \\
\hline Walk with stick & $29(67.3 \%)$ & $32(78.1 \%)$ \\
\hline \multicolumn{3}{|l|}{ Motor Characteristics } \\
\hline Berg Balance Scale (0-56), mean (SD) & $45.5(11.6)$ & $43.5(8.9)$ \\
\hline Rivermead Mobility Index (0-15), mean (SD) & $11.6(2.5)$ & $11.3(2.7)$ \\
\hline Fugl-Mayer Assessment (0-34), mean (SD) & $23.5(8.0)$ & $22.2(8.1)^{*}$ \\
\hline \multicolumn{3}{|l|}{ Cognitive Characteristics } \\
\hline Montreal Cognitive Assessment (0-30, mean (SD) & $24.7(4.2)$ & $23.2(6.2)$ \\
\hline \multicolumn{3}{|l|}{ Conscious motor control preference } \\
\hline Movement Specific Reinvestment Scale (0-10), mean (SD) & $4.9(2.5)$ & $5.1(2.6)$ \\
\hline
\end{tabular}

* Due to fatigue one participant from the explicit group was unable to complete the Fugl-Mayer Assessment 


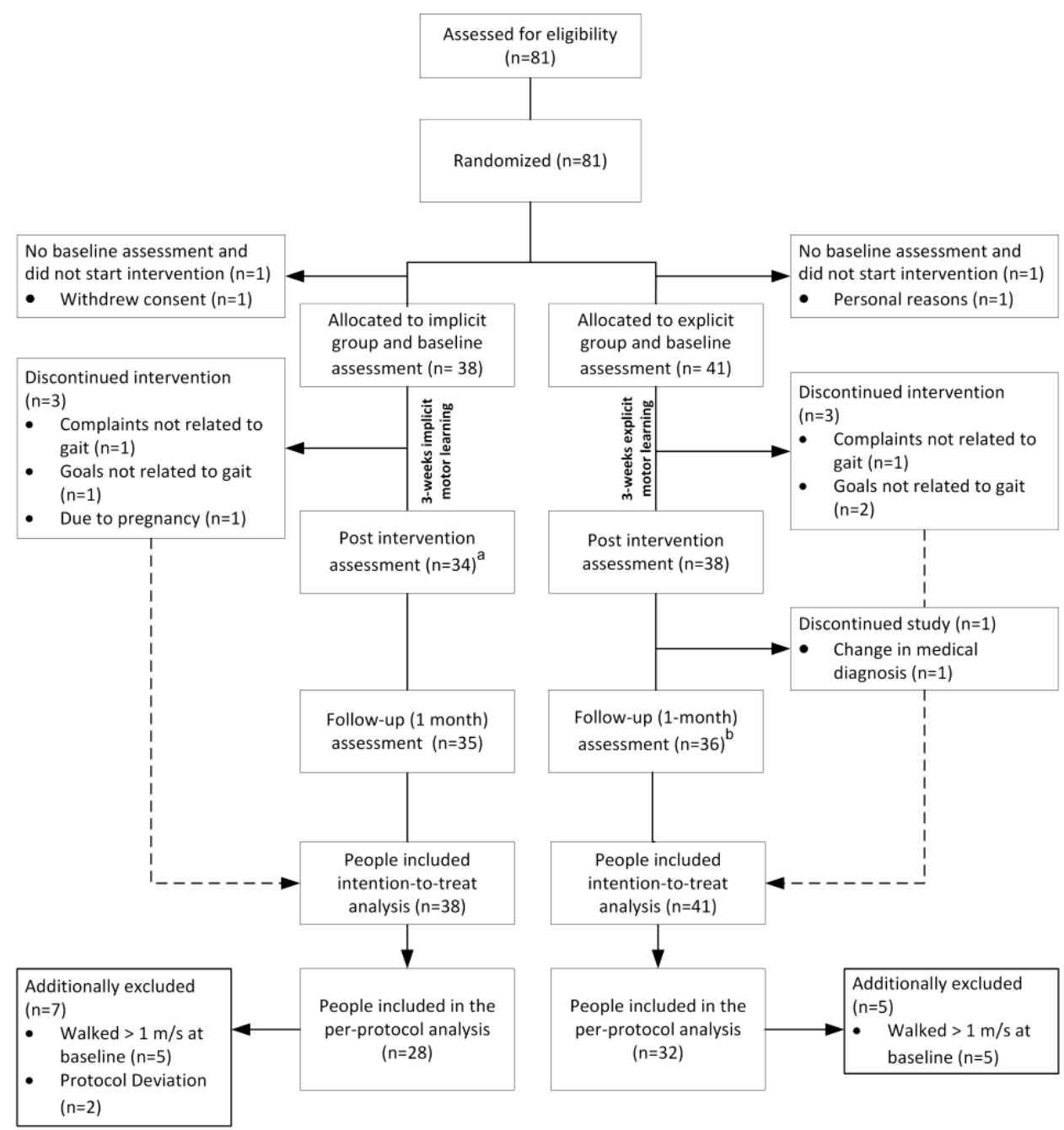

Figure 2. Flowchart of the trial.

a One participant was unavailable for the post intervention assessment $(n=1)$ b One participant was unavailable for the follow-up assessment $(n=1)$

\section{Results of the intention-to-treat analysis}

Table 2 presents the observed means (SD) per group and time point, the within-group differences, and the estimated between-group differences of the implicit versus the explicit group. Mixed linear models revealed no statistically significant differences between the groups immediately after (difference estimate $0.02 \mathrm{~m} / \mathrm{s}$ [95\% CI -0.04 to 0.08 ], $p=0.498$ ) and one-month post intervention (difference estimate $-0.02 \mathrm{~m} / \mathrm{s}$ [95\% CI -0.09 to 0.05 ], $p=0,563$; see table 2 and figure 3). Also, no clinically relevant (MCID: $0.16 \mathrm{~m} / \mathrm{s}$ ) difference between groups were observed.107 

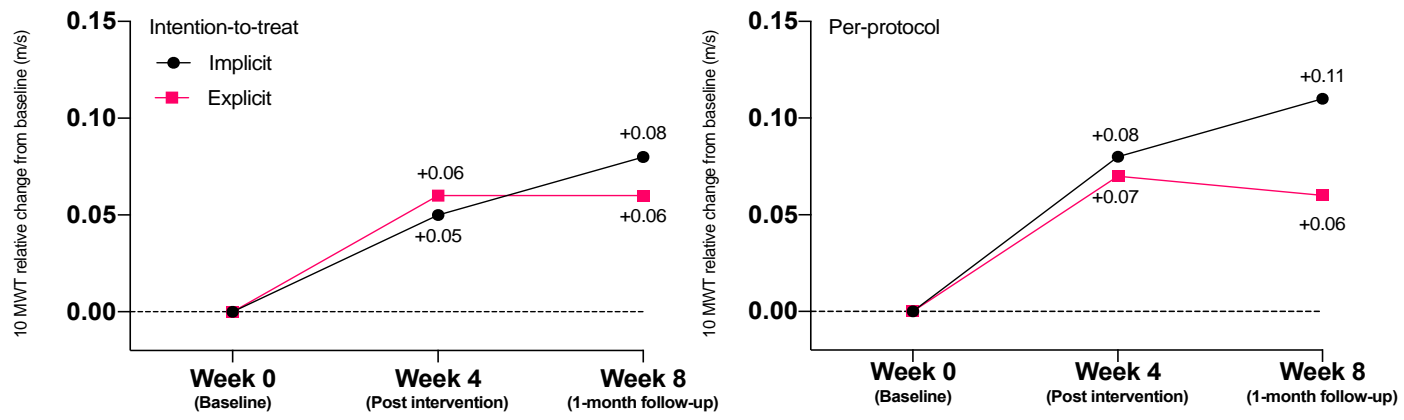

Figure 3. Performance of the 10 Meter Walking Test.

No statistically significant differences in favour of any group was obtained on any of the other secondary outcome parameters (table 2). Over time, within groups, positive changes were observed in outcome measures related to gait function (DGI, DTEmotor), and quality of life (SAQOL-39). Participants' perceived effects with regard to the intervention was similar for both groups (see figure 4). With regard to the verbal protocol, on average, people in the implicit group accumulated significantly fewer explicit rules $(M=0.38, R=0$ to 2 rules, $S E=0.10)$ compared to the explicit group $(M=2.42, R=0$ to 6 rules, $S E=0.27)$, $\mathrm{t}(68)=-7.07, p<.05$ after the intervention.

\section{To which extent did your walking \\ ability change over the last three weeks?}

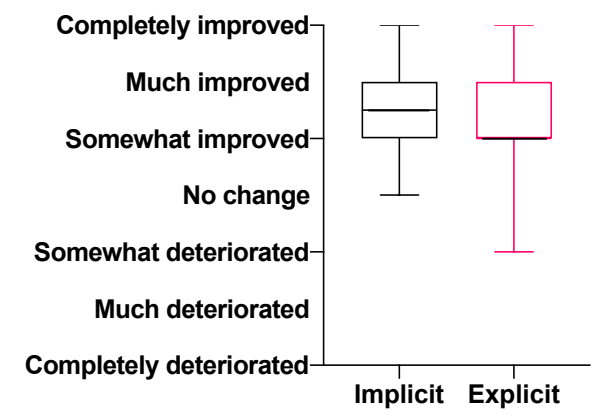

Figure 4. Boxplot of the Global Perceived Effect.

Sub-group analysis on cognition

In total 15 people (implicit group $n=5$; explicit group $n=10$ ) had a MoCA score $\leq 21$. No trend in favour of the implicit intervention was observed in the descriptive sub-group analysis on cognition (see supplementary data 1). 


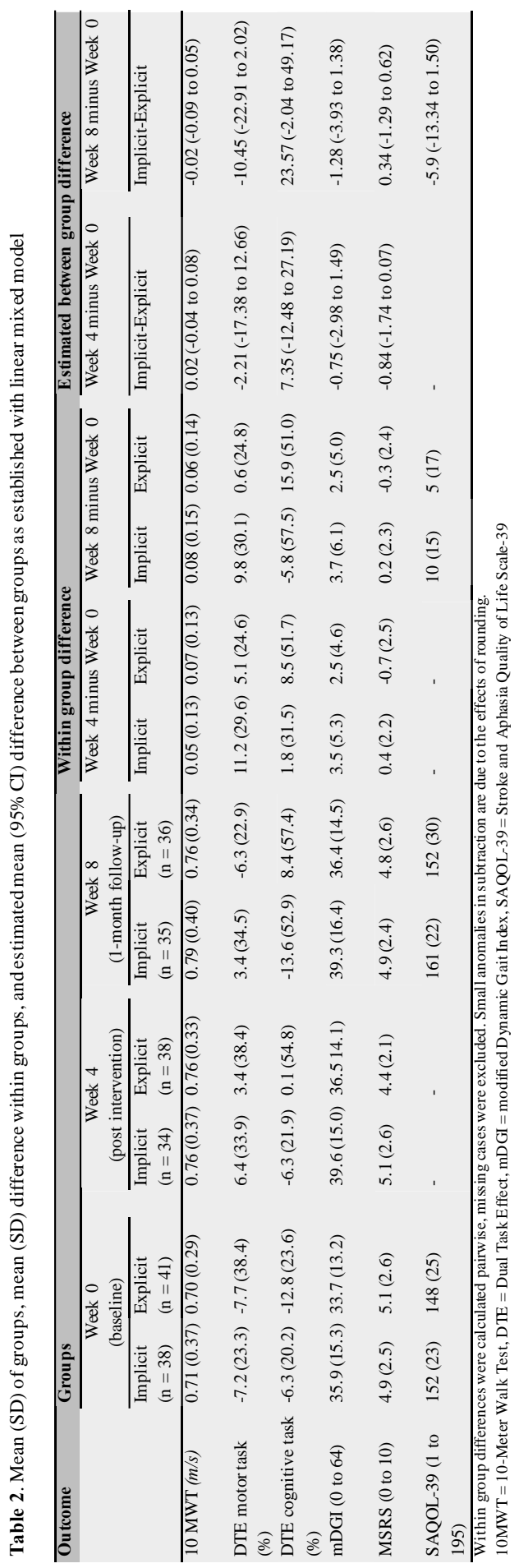




\section{Results of the Per-Protocol}

The per-protocol analyses led to slightly larger changes between groups but again did not lead to statistically significant after (difference estimate $-0.06 \mathrm{~m} / \mathrm{s}$ [ $95 \% \mathrm{CI}-0.13$ to 0.02 ], $p=0.140$ ) or clinically relevant effects (MCID: $0.16 \mathrm{~m} / \mathrm{s}$ ) between group on the primary outcome (see right graph figure 3 and supplementary data 2).

\section{DISCUSSION}

People after stroke in the chronic phase of recovery who received an implicit motor learning walking intervention (analogies) under the guidance of physiotherapists in their home environments, had similar effects on walking speed compared to those who received an explicit motor learning walking intervention (verbal detailed instructions). No statistically nor clinically relevant differences between groups were found as between group differences (intention-to-treat $\Delta 0.02 \mathrm{~m} / \mathrm{s}$; per-protocol $\Delta 0.05 \mathrm{~m} / \mathrm{s}$ ) did not exceed the chosen clinically relevant threshold of $0.16 \mathrm{~m} / \mathrm{s} .107$ Similarly, based on the descriptive sub-group analysis on cognition (MoCA $\leq 21$ ) no trend in favour of the implicit intervention was observed (table 3 ). No statistical sub-group analysis on cognition was performed based on small group sizes.

To our knowledge this was the first and largest trial in the field of stroke rehabilitation to examine the effectiveness of implicit motor learning to improve the functional 'walking' task within a clinically relevant context (home environment of the patient).91 The results of this study did not replicate the more promising findings on implicit motor learning in stroke from earlier studies, generally performed in more standardized, laboratory settings and/or with non-functional tasks e.g.23,29,137. A variety of factors related to the selection of participants (selection bias), use of the 10MWT as primary outcome measure (information bias) and operationalization of the intervention (contrasts) may have influenced the results and led to these neutral findings.

First, a selection bias may have occurred. To increase generalizability of the results and to gain a better insight into the potential effects in clinical practice, we chose to include a sample of stroke patients, which reflects the heterogeneity of the stroke population as seen in rehabilitation. The researched target population group therefore showed a large variability in terms of demographics as well as physical and cognitive abilities. This heterogeneity may reflect reality in practice but might also have diminished the results. Further, the erroneous inclusion of ten participants whose baseline walking speed exceeded the inclusion criterion may have led to a ceiling effect. This ceiling effect might explain the larger, but not significant, trend towards implicit motor learning (see figure 3 lower graph) in the per-protocol analysis. In addition, the study was probably underpowered due to this deviation. 
Second, the use of the 10MWT as primary outcome measure may have implications for both the findings themselves and the interpretation in terms of clinical meaningfulness. The 10MWT was chosen as the primary outcome measure due to its validity, reliability and feasibility within clinical practice 138 but also to allow comparison with other studies.139 The advantage of using walking speed as a primary outcome is the integrated result on multiple gait parameters such as step length and frequency 133,134 and the direct relation to changes in functional scale.114 However, it could be argued that the 10MWT might not have been sensitive enough to detect changes due to implicit learning if those underlying changes are small or not obviously related to walking speed (e.g. improvement of confidence during walking).

Another explanation for the neutral results could originate from the way the interventions were operationalised. Contrary to earlier studies in more controlled settings and with nonfunctional tasks such as serial reaction time tasks,91 it seems difficult to keep the contrast between interventions equally large when including a functional task within a clinically relevant environment. In contrast to other studies 10,11 the exact number of rules were not pre-defined but tailored to the participants. For example, Lam et al used a fixed number of 'eight' verbal rules compared to 'one' analogy.11 The provided number of explicit rules (explicit intervention) may have been limited because of ethical reasons, potentially resulting in a diminished contrast between groups.

Within this study we assessed the implicit nature of the intervention by asking participants to report the number of explicit rules the learned (verbal protocol), assessing durability of performance over a longer time period and dual task interference.10,11 None of these measures revealed a clear picture on the nature of the learning process. For instance, fewer rules were accumulated in the implicit compared to the explicit group, but it remains unclear whether these rules have been acquired through treatments before enrolment of this study. In addition, for some participants the tone-counting task may have been too easy not leading to dual task interference, whereas for other people the task was too difficult. Due to this large variation in performance on the cognitive (tone counting) dual task it was not possible to further legitimately interpret these results.

Finally, on average both groups slightly improved their walking speed after the intervention $(+0.08 \mathrm{~m} / \mathrm{s}$ in the implicit group and $+0.06 \mathrm{~m} / \mathrm{s}$ in the explicit group) exceeding the threshold for clinical relevant change of $>0.06 \mathrm{~m} / \mathrm{s}$ for within group differences as established by Perera et al.140 It might be that using implicit or explicit motor learning does not make a (clinically relevant) difference for the results of walking rehabilitation within the included target group and setting of this trial. It is remarkable that the detected improvement (in both groups) remained relatively stable at the follow-up test. This finding might be seen as a form of retention and indicates that motor learning occurred rather than just a temporal improvement in motor performance. 


\section{Future research}

The design of this RCT was carefully prepared by research into underlying theories, 4 feasibility and piloting testing24,68 of implicit motor learning.30 Applying the RCT in its cleanest form in clinical settings 141 and with complex interventions was challenging as we needed to balance between external validity (generalizability of the results for daily practice) and internal validity (standardization and reliability of the results). Other designs may be considered to evaluate effectiveness of long-term, highly individualized, and complex interventions, 30 as needed in the field of motor learning. Two recent studies suggest that tailoring motor learning interventions towards patient characteristics and preferences might be important, promoting more pragmatic trials. 86,132 The interventions may also be applicable for people with more severe cognitive impairments (MoCA $\leq 21$ ) as equal trends in performance were found within this sub-group. A logical next step would be to assess which patient characteristics influence motor learning interventions and how these factors influence the learning process. Therefore, cohort studies in which all potential influencing factors (e.g. activity dependent plasticity, cognition, or individual preferences) are measured over time and therapist document the used motor learning approach in detail might be an interesting alternative to consider.

To gain more insight in the gait mechanisms and functional effects when applying implicit motor learning, future studies may consider combining upcoming instruments for quantitative gait analysis which can be performed outside laboratory settings (e.g. use of wearable sensors) 142,143 with patient specific outcome measures which can detect functional relevant changes within individualized goals (e.g., Patient Specific Functional Scale).144,145

\section{Conclusion and clinical message}

In this study, no overall benefits of implicit motor learning over explicit motor learning for improving walking performance in people after stroke in the chronic phase of recovery were found. The treatment effects in this study may have been diluted by "noise" accompanied with research within real life settings, complex tasks and a representative sample of the target population. For tailored motor learning approaches more insight is needed on the patient characteristics and preferences that influence the process of motor learning. While awaiting further results, therapists may consider both motor learning approaches to facilitate walking speed within the stroke population. 


\section{Acknowledgements}

The authors would like to thank the therapists, and researchers who contributed in conducting the trial: Jos Halfens, Jos Kurvers, Wiel Regtop, Kyra Theunissen, Monique Rothbauer, Inge de Lang, Ritch te Kampen, Renée van den Heuvel, Joep Westbroek. Furthermore, we thank the client participants Nathalie Sieben, Else de Bont, and Anja Minheere for their perspectives and thoughts on the trial during the set-up of the study. We thank Peter Konsten for his support on the assessment forms and Bjorn Winkens for his advice in the data analyses. Finally, we thank all participants that took part in the study. A pre-print of this work is available at medRxiv.146

\section{Funding}

This work was supported by Nationaal Regieorgaan Praktijkgericht Onderzoek SIA (RAAKPRO; grant number 2014-01-49PRO).

\section{Conflicts of interest}

Non declared. 


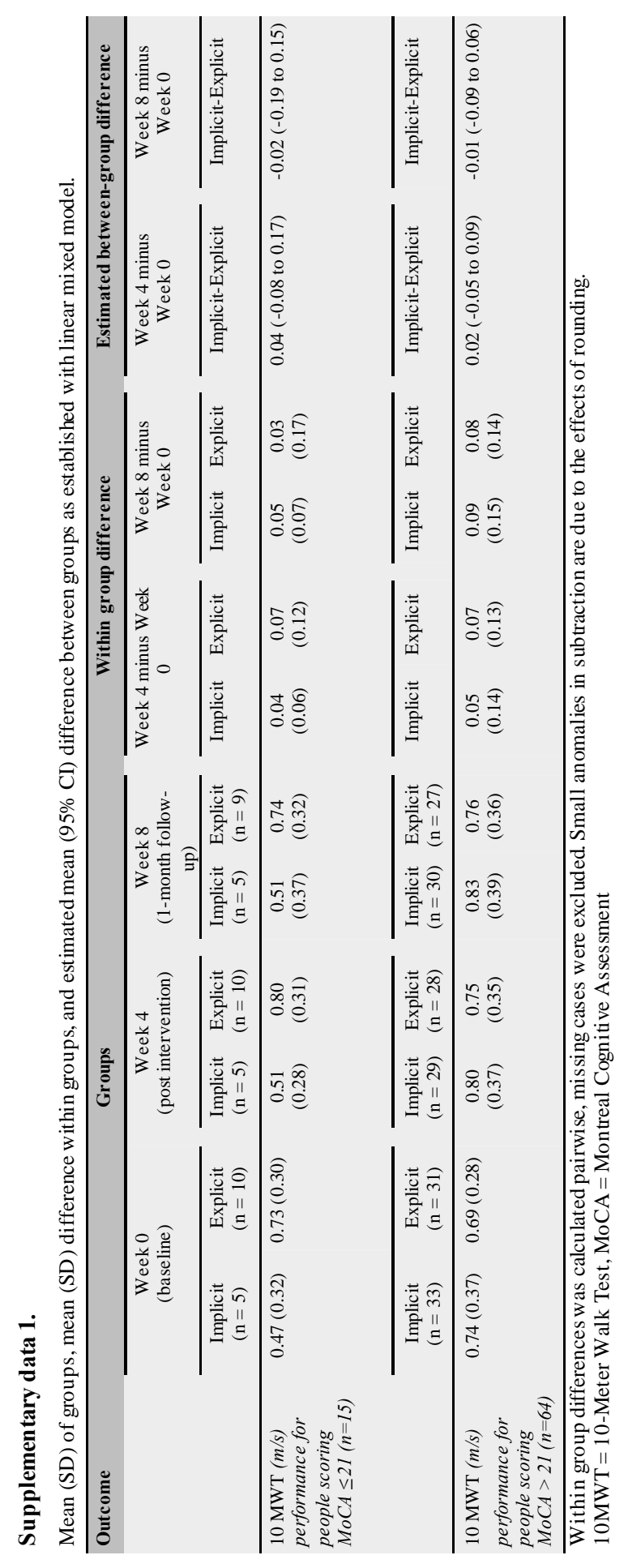




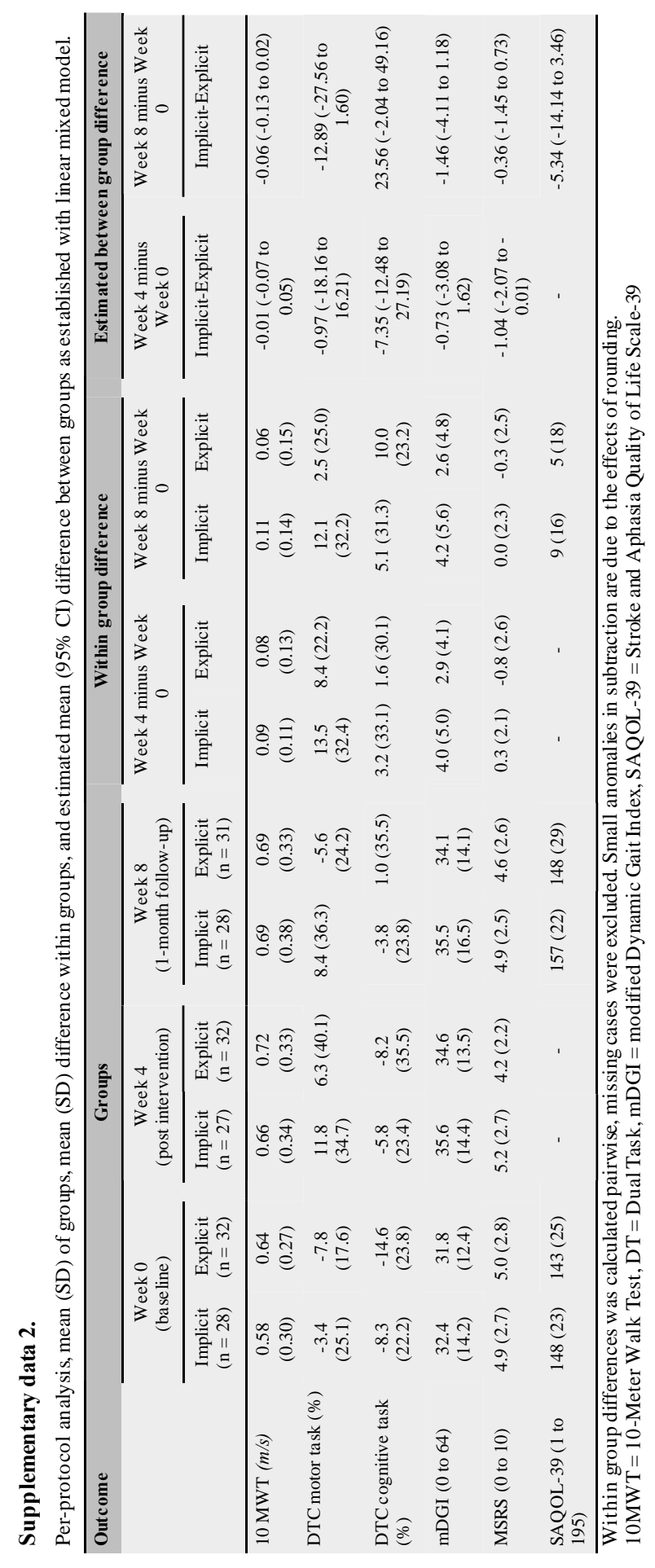




\section{CHAPTER 6}

\section{Implicit and explicit motor learning interventions for gait in people after stroke: a process evaluation on fidelity and feasibility performed alongside a randomized controlled trial}

Li-Juan Jie, Melanie Kleynen, Kenneth Meijer, Anna JHM Beurskens, Susy M Braun

Submitted, available as pre-print form as:

Jie, L. J., Kleynen, M., Beurskens, A., Meijer, K., \& Braun, S. (2019). Implicit and explicit motor learning interventions have similar effects on walking speed in people after stroke: a randomized controlled single blind trial. medRxiv. doi: 10.1101/2020.01.17.20017897

(C) The Authors 2020. This work is licensed under the Creative Commons Attribution 4.0 International License. http://creativecommons.org/licenses/by/4.0/ 


\begin{abstract}
Purpose: To evaluate whether the implicit and explicit motor learning walking interventions for people after stroke delivered in a randomized controlled trial were performed as intended (fidelity) and to report the therapist and participant experiences with regard to feasibility.
\end{abstract}

Methods: Dose delivered and content of instructions were assessed by evaluating the therapist logs and audio recordings of the training sessions. The therapist and participant experiences were assessed by means of self-developed questionnaires.

Results: 79 people were included of which seven people (9\%) dropped out. The remaining participants all received the required minimum of seven sessions. Overall therapists adhered to the intervention guideline. On average 5.2 and 0.4 explicit rules were used within the explicit group and implicit group respectively. Therapists and participants were generally positive about the feasibility but frequent comments were made by the therapists regarding "therapy time restrictions" and "tendency of the participants to develop explicit rules". A variety of changes were observed ranging from improvements in spatiotemporal parameters to cognition and emotions.

Conclusion: The delivery of the implicit and explicit motor learning walking interventions were successful in terms of fidelity. Therapists and participants were generally positive about the feasibility of the intervention.

82 | Process evaluation of the Randomised Controlled Trial 


\section{INTRODUCTION}

Within stroke rehabilitation gait is one of the most practiced activities.131 One way to apply over ground gait training is to use an explicit or implicit motor learning approach. Explicit learning is typically referred to as a more conscious approach to learning, 4 in which many detailed instructions about the movement itself are provided to the learner. Whereas implicit learning strives to takes place in a more automatic manner, without much knowledge of the underlying facts and rules of the movement. 4 To apply implicit motor learning different learning strategies can be used, for example errorless learning, dual task learning or analogy learning. In analogy learning one single analogical rule that integrates all (explicit) knowledge to perform the to-be-learned motor skill is used. In current clinical practice physiotherapists tend to use primarily a large amount of verbal instructions (explicit learning) or use a mix of implicit and explicit motor learning approaches.6,130 However, within sport advantages and positive results of implicit motor learning over explicit motor learning have been found, such as: better performance while multitasking or in pressurised environments and stable performance over a longer time period. 8 These positive results have the potential to transfer to the therapeutic environment.

To date the majority of studies on the effectiveness of implicit motor learning within the stroke population have been performed within laboratory settings and with non-functional computer tasks.91 Only few pilot and feasibility studies have been performed within clinical practice using real life tasks. These studies demonstrate the feasibility and potential effects of implicit motor learning within neurological rehabilitation.24,68 Given the limited evidence, a large randomized controlled trial 146 (preprint version) including a total of 79 participants was conducted, in which implicit and explicit walking interventions for people after stroke were compared. We hypothesized that implicit motor learning would result in greater improvements of walking speed post intervention.146 It was found that participants in both intervention groups improved with little to no differences between the interventions. To our knowledge this was the first study that took place within a clinical setting and with personalised interventions. No rigid protocol was implemented, however a framework was provided for the implicit and explicit interventions. The process evaluation took place parallel to the randomized controlled trial and covered the following research questions:

- To what extent did the therapists deliver the interventions as intended (fidelity)?

- How did the therapist's experience delivering the interventions with regard to the feasibility?

- How did the patients' experience the intervention with regard to the feasibility? 


\section{METHOD}

In this prospective process evaluation both quantitative and qualitative methods were used. The protocol of the randomized controlled trial 135 was approved by the local Ethics committee METC-Z in Heerlen, the Netherlands (Ethics nr: 17-T-06) and registered in the Netherlands Trial Register (Trial NL6133 (NTR6272)).

\section{Participants, and therapists}

Both the participants of the study and therapists who provided the interventions were included in the process evaluation. The participants were recruited through local private practices, rehabilitation institutes, and a local health-related newspaper. All the participants evaluated their experience about the received intervention through a participant questionnaire developed to be specific to the intervention.

The therapists were recruited through the (professional) network of the researchers. Only experienced therapists ( $>10$ years) within neurological rehabilitation and/or experts within the field of the motor learning were included in the study. All therapists provided both the implicit and explicit interventions.

\section{Interventions, training of therapists and assessors}

Within this RCT an implicit and explicit motor learning walking intervention for people after stroke was evaluated. Based on scientific evidence 4,24,68,132 and expert experiences (practice based evidence) an intervention guideline was developed that outlined precisely how the treatments (implicit and explicit conditions) were supposed to be delivered. The guideline for both interventions was comparable in terms of dose (three sessions per week over a 3-week period) and intensity (30 minutes per session). A minimum frequency of seven sessions was considered as being consistent with the protocol.135 Outside guided therapy and after the 3-week intervention period participants were asked to use the practiced instructions in daily life. First the treating therapist identified the individual walking problem (similar for both groups), after which tailored instructions were provided in either an implicit or explicit manner according to the intervention guideline. Figure 1 provides an overview of the main characteristics of the interventions. 


\begin{tabular}{|c|c|c|}
\hline Elements & Implicit condition & Explicit condition \\
\hline General rules & $\begin{array}{l}\text { - Minimal use of verbal instructions. } \\
\text { - For the participant, learning should } \\
\text { progress without being aware of the } \\
\text { underlying gait parameters that the } \\
\text { therapist aims to improve. }\end{array}$ & $\begin{array}{l}\text { - Many verbalinstruc tions should be } \\
\text { used, however, only to an extent that } \\
\text { is feasible for the participant. } \\
\text { - For the participant, leaming should } \\
\text { progress in a conscious manner. } \\
\text { The participant should be very } \\
\text { aware of the underlying gait } \\
\text { parameters that the therapist aims to } \\
\text { improve. }\end{array}$ \\
\hline \multirow[t]{2}{*}{ Instructions } & $\begin{array}{l}\text { The instructions should only contain } \\
\text { information about the analogy. The analogy } \\
\text { can be based on: } \\
\text { - Past experiences and/ormeaningful } \\
\text { situations. It should contain an image. }\end{array}$ & $\begin{array}{l}\text { Instructions should contain: } \\
\text { - Detailed instructions with reference } \\
\text { to joints, angles, and precise placing } \\
\text { of extremities/limbs. } \\
\text { - Biomechanical information. } \\
\text { - } \quad \text { Keep intemally focussed (directed } \\
\text { on body movements). } \\
\text { - Create a conscious walking } \\
\text { environment. } \\
\text { - Frequently repeat verbal } \\
\text { instructions. }\end{array}$ \\
\hline & $\begin{array}{l}\text { - No information about joints, angles, } \\
\text { position of limbs, etc. } \\
\text { - No details about spatiotemporal gait } \\
\text { parameters or biomec hanics of gait. }\end{array}$ & $\begin{array}{l}\text { No information about how the } \\
\text { movement feels, no use of images, } \\
\text { no reference to familiarsituations, } \\
\text { no imagery. }\end{array}$ \\
\hline Feedback & $\begin{array}{l}\text { - Knowledge of results (state goal of } \\
\text { movement). } \\
\text { - Link the feedback to the analogy e.g. } \\
\text { situations, images, or emotions. } \\
\text { - Use positive feedback. }\end{array}$ & 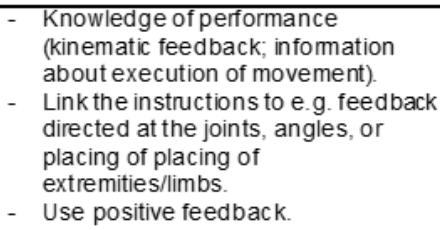 \\
\hline Examples & $\begin{array}{l}\text { - Walk as if you are walking through a } \\
\text { deep layer of snow. } \\
\text { - Walk as if you follow the footprints in the } \\
\text { sand. } \\
\text { - Try to cross a small bridge. }\end{array}$ & $\begin{array}{l}\text { - } \text { Place yourheel right. } \\
\text { - } \quad \text { Lift your feet. } \\
\text { - } \\
\text { Transferyour weight on your } \\
\text { - } \text { Push off leg. } \\
\text { - } \text { Bend yourknees. } \\
\text { - } \text { Walk up-right. } \\
\text { - } \quad \text { Lift yourwalking frame. }\end{array}$ \\
\hline
\end{tabular}

Figure 1. Overview with the main characteristics of the interventions. Adapted from Jie et al.135 


\section{The implicit intervention}

Within the implicit intervention we aimed to influence the walking pattern by facilitating the learning process with no or minimal increase of verbal knowledge.4 Analogy learning was chosen to guide the implicit intervention. In analogy learning, complex information e.g. step-by-step rules (explicit knowledge) about the motor skill, is replaced by one simple biomechanical metaphor or analogy.11 Based on the walking problem and participant preferences an analogy was created, practiced and adjusted if necessary, e.g. if the analogy did not lead to the desired adaptations of the movement performance.

\section{The explicit intervention}

Within the explicit intervention the opposite learning environment was created as much as possible, by organizing a conscious learning environment in which many verbal explicit instructions (explicit knowledge) were used. Although the explicit intervention strived to use as many instructions as appropriate, the number of instructions were used up to an amount that was considered to be possible (feasible) for the participants to process/handle. The latter was based upon as the judgement of the treating therapists.

To standardize both interventions, the therapists were trained during five sessions prior to the study. During these sessions the intervention guideline was discussed, and example cases were used to practice the application of the interventions. During the study three evaluation sessions took place to evaluate experiences or discuss difficulties and to ensure all therapists were using the intervention guideline in a similar manner.

\section{Data collection}

Demographic information of the participants such as age, gender and time post stroke was gathered at baseline. The following clinical characteristics were assessed before the start of the interventions: cognitive level (Montreal Cognitive Assessment),78 static balance and fall risk (Berg Balance Scale), 108 mobility disability (Rivermead Mobility Index),109 ability to make movements outside the synergetic patterns (Fugl-Meyer assessment of the lower limb),110 and propensity of reinvestment (Movement Specific Reinvestment Scale).111,112 Table 1 presents an overview of the different measures, timing of the measurement assessments, number of participants who were received and completed the measurements that were used within the process evaluation. 
Table 1. Overview of the different measures used to answer the research questions.

\begin{tabular}{|c|c|c|c|c|c|}
\hline Measure & $\begin{array}{l}\text { Process } \\
\text { variable }\end{array}$ & $\begin{array}{l}\text { Timing of } \\
\text { measure }\end{array}$ & Completed & $\begin{array}{l}\text { Response } \\
\text { rate }\end{array}$ & Comments \\
\hline $\begin{array}{l}\text { Therapist logs } \\
\text { (N Treated: } 79 \text { ) }\end{array}$ & Fidelity & Week 1-3 & $n=72$ & $91 \%$ & $\begin{array}{l}7 \text { people } \\
\text { dropped-out } \\
\text { during the } \\
\text { intervention }\end{array}$ \\
\hline $\begin{array}{l}\text { Audio Recordings } \\
\text { Intended: } 10 \\
\text { interventions } \\
5 \text { implicit } \\
\text { interventions } \\
5 \text { explicit } \\
\text { interventions }\end{array}$ & Fidelity & Week 1-3 & $n=10$ & $100 \%$ & \\
\hline $\begin{array}{l}\text { Therapist } \\
\text { Questionnaire } \\
\text { (N Handed: 72) }\end{array}$ & $\begin{array}{l}\text { Therapist } \\
\text { experiences }\end{array}$ & Week 3 & $n=72$ & $100 \%$ & \\
\hline $\begin{array}{l}\text { Patient } \\
\text { Questionnaire } \\
\text { (N Handed: 72) }\end{array}$ & $\begin{array}{l}\text { Participant } \\
\text { experiences }\end{array}$ & Week 3 & $n=72$ & $100 \%$ & \\
\hline
\end{tabular}

\section{Fidelity}

Within this process evaluation fidelity was assessed with regard to the dose (number of sessions) and content of instructions (adherence to the intervention guideline and the number of explicit rules provided). Data from the therapist logs, and audio files were used for this purpose. Therapist logs were used to evaluate therapists adherence with the intervention guideline.10 In the logs the number of sessions delivered, the provided instructions and any deviations from the protocol were reported. In addition to the selfreported data (subjective) a random selection of 10 gait training sessions ( 5 implicit and 5 explicit interventions) were audio-recorded.

\section{Therapist experiences with regard to feasibility}

The therapist questionnaire included four questions, one question each to explore their thoughts on the intervention period (3-weeks in total), duration of the training sessions (30 minutes per session), the amount of sessions ( 9 in total) and future use of the learned strategy by the participant. The second part of the therapist questionnaire included four questions related to independent practice of the participants, suitability of the interventions, the tendency to develop explicit rules, and any observed changes or improvements in gait. The first four questions were recorded using binary outcomes 'yes/no', whereas the last four questions were scored on a 11-point Likert scale. There was space for free comments to elaborate on each response (see table 3 for questions). 


\section{Patient experiences with regard to feasibility}

The patient questionnaire was administered at the end of the nine training sessions. Participants evaluated the following three questions: 1) the ease-of-use of the provided instructions 2) their gained confidence in walking and 3) their overall experience with regard to the received gait training sessions. Each participant scored their satisfaction about the intervention on an 11-point Likert scale (see table 5 for the questions).

\section{Data analysis}

Data from the therapist logs (dose delivered, provided instructions), audio recordings (provided instructions), therapist- (feasibility) and participant- (feasibility) questionnaires were extracted by a research assistant and summarised in an excel spreadsheet. The quantitative outcomes of the questionnaires were presented using descriptive statistics (median, IQR). Frequently reported responses were described and quotes were used as clarifying examples.

The actual dose delivered was determined by counting the number of completed sessions within the therapist logs. The content (provided instructions) of the therapist logs and audio recordings were examined for the presence of explicit rules in both groups and the use of analogies within the explicit intervention by two independent raters.135 To determine contrast between groups the number of explicit rules per participant was counted. To explore whether cognition influenced the quantity of explicit rules, the number of explicit rules were counted in people with lower cognitive abilities (MoCA $\leq$ 21) and vice versa. If explicit rules were used within the implicit intervention or when analogies were used within the explicit intervention this was reported as deviations from the protocol if they occurred in two or more sessions per participant.135 To provide more insight in the content of the given instructions, an overview of examples within the implicit (analogy instructions) and explicit (explicit rules) interventions were presented.

\section{RESULTS}

In total 79 people after stroke were included in the main analyses of the study. During the intervention period seven participants $(9 \%)$ dropped out (implicit group $n=3$; explicit group $n=4$ ). Three people wanted to improve overall fitness but did not have specific goals related to gait and two participants had other complaints not related to gait and therefore discontinued with the intervention. Furthermore, one participant stopped due to pregnancy and another person due to a change in medical diagnoses. These participants and their therapists did not evaluate the intervention (therapist and patient logs). Therefore, a total of 72 cases were included and evaluated in the process evaluation (Table 2). Based upon the three evaluation sessions with the treating therapists no deviation in the application of the intervention guideline were reported. 
Table 2. Baseline characteristics of the participants $(n=72)$.

\begin{tabular}{|c|c|c|}
\hline & $\begin{array}{l}\text { Implicit } \\
(n=35)\end{array}$ & $\begin{array}{l}\text { Explicit } \\
(n=37)\end{array}$ \\
\hline \multicolumn{3}{|l|}{ General Characteristics } \\
\hline Age $(y r)$, mean (SD) & $64.6(9.4)$ & $67.8(11.6)$ \\
\hline Sex, n males (\%) & $24(63 \%)$ & $25(61 \%)$ \\
\hline Height $(\mathrm{cm})$, mean (SD) & $171.7(8.0)$ & $172.0(8.8)$ \\
\hline \multicolumn{3}{|l|}{ Educational level, n (\%) } \\
\hline Elementary education & $0(0 \%)$ & $1(2 \%)$ \\
\hline Secondary education & $20(53 \%)$ & $18(44 \%)$ \\
\hline Vocational training & $10(26 \%)$ & $11(27 \%)$ \\
\hline University & $8(21 \%)$ & $11(27 \%)$ \\
\hline \multicolumn{3}{|l|}{ Stroke Characteristics } \\
\hline Time post stroke (months), mean (SD) & $72.8(59.3)$ & $67.5(69.1)$ \\
\hline Side of stroke, $\mathrm{n}$ right $(\%)$ & $19(50 \%)$ & $16(19 \%$ \\
\hline \multicolumn{3}{|l|}{ Independent walking, $\mathrm{n}(\%)$} \\
\hline Walk unaided & $9(24 \%)$ & $9(22 \%)$ \\
\hline Walk with stick & $29(67 \%)$ & $32(78 \%)$ \\
\hline \multicolumn{3}{|l|}{ Motor Characteristics } \\
\hline Berg Balance Scale (0-56), mean (SD) & $45.5(11.6)$ & $43.5(8.9)$ \\
\hline Rivermead Mobility Index (0-15), mean (SD) & $11.6(2.5)$ & $11.3(2.7)$ \\
\hline Fugl-Meyer Assessment (0-34), mean (SD) & $23.5(8.0)$ & $22.2(8.1)^{*}$ \\
\hline \multicolumn{3}{|l|}{ Cognitive Characteristics } \\
\hline Montreal Cognitive Assessment (points/30, mean (SD) & $24.7(4.2)$ & $23.2(6.2)$ \\
\hline \multicolumn{3}{|l|}{ Conscious motor control preference } \\
\hline Movement Specific Reinvestment Scale (0-10), mean (SD) & $4.9(2.5)$ & $5.1(2.6)$ \\
\hline
\end{tabular}

* due to fatigue one participant from the explicit group was unable to complete the Fugl-Meyer assessment

\section{Fidelity}

In terms of dose delivered the therapist's logs revealed that in total 627 therapy sessions were delivered (303 implicit sessions; 324 explicit sessions). All 72 participants (100\%) attended seven or more sessions according to protocol, of which 55 participants $(76 \%)$ attended all nine, 13 participants (18\%) completed eight and four participants $(6 \%)$ completed seven training sessions.

In $4(1 \%)$ of the 324 sessions within the explicit intervention, an analogy (implicit instruction) was used. In 14 (5\%) of the 303 sessions within the implicit intervention, 
explicit rules were used. Two protocol deviations were observed within the implicit intervention i.e. explicit rules that were used in $\geq 2$ sessions per participant. No protocol deviations were observed within the explicit condition. However, in one occasion, no explicit rules were used while being allocated to the explicit intervention. For this case the therapist log revealed that more general instructions were provided such as accelerate, decelerate or walk and look right, but these were not counted as explicit rules as they did not comply to the definition of explicit rules as defined within research protocolio.

Therapist logs revealed that on average $\mathrm{M}=5.2$ (Range $=0$ - 10) explicit rules were used within explicit group and $\mathrm{M}=0.4$ (Range $=0-3$ ) explicit rules the implicit group. Within the explicit group, on average less explicit rules were used $(M=4.1, R=2-8, n=10)$ when participants were more cognitively impaired (MoCA $<21$ ), compared to those who were not $(M=5.6, R=0-10, n=27)$. Table 3 provides an overview of the used instructions within the implicit and explicit group.

Table 3. Examples of the provided instructions by the therapists.

\begin{tabular}{l} 
Implicit group \\
\hline - $\quad$ Walk as if you are cross-country skiing \\
- $\quad$ Walk on the rhythm of the song Africa by Toto \\
- Do as if you are kicking the ball in front of you (football) \\
- $\quad$ Do as if the wind pushed you forward while walking \\
\hline Explicit group \\
- $\quad$ Stretch the hip while walking \\
- $\quad$ Push off with your toes \\
- $\quad$ Place your cane on the side, not in front of you \\
- $\quad$ Bend you knees during stance phase \\
- $\quad$ Stretch the hip while walking
\end{tabular}

\section{Therapist experiences with regard to feasibility}

In table 3 subjective responses of the therapists related to the feasibility and observed changes of the intervention are presented. In the therapist questionnaire no apparent differences between the groups were visible (table 4). Comments were frequently made with regard to "time restrictions", "tendency to develop explicit rules" and "observed changes". 


\section{Time restrictions}

Despite good adherence to the protocol (questions 1 to 3), therapists in both groups mentioned more time was preferred (> nine sessions and > 30 minutes per session) when participants were aphasic. Furthermore, when therapists provided the implicit intervention, they mentioned that sometimes more than one therapy session was preferred to establish a personalized metaphor. For example, therapists mentioned "I needed the first four sessions find the right approach. With music, and rhythm music I experienced good results. Three extra sessions would have been effective for him" (implicit intervention, therapist 3), or "The first session, 30 minutes were tide. Later on, 30 minutes seemed sufficient" (implicit intervention, therapist 3).

Table 4. Responses of the therapist questions about their experiences and observed changes $(n=72)$.

\begin{tabular}{|c|c|c|}
\hline Questions & Intervention & \% Agreed \\
\hline \multirow{2}{*}{$\begin{array}{l}\text { Were you able to adhere to the framework as } \\
\text { intended (content wise)? }\end{array}$} & Implicit & $91 \%$ \\
\hline & Explicit & $97 \%$ \\
\hline \multirow{2}{*}{$\begin{array}{l}\text { Were you able to finish the gait trainings within } 30 \\
\text { minutes as described in the protocol? }\end{array}$} & Implicit & $97 \%$ \\
\hline & Explicit & $97 \%$ \\
\hline \multirow{2}{*}{$\begin{array}{l}\text { Were the } 9 \text { training sessions sufficient to complete } \\
\text { the overall training according to protocol? }\end{array}$} & Implicit & $91 \%$ \\
\hline & Explicit & $95 \%$ \\
\hline \multirow{3}{*}{$\begin{array}{l}\text { Do you think that the participant will keep using the } \\
\text { learned strategy in the future? (Yes / No) }\end{array}$} & Implicit & $77 \%$ \\
\hline & Explicit & $87 \%$ \\
\hline & & Median (IQR) \\
\hline \multirow{2}{*}{$\begin{array}{l}\text { Did the participant practice independently outside } \\
\text { the supervised training sessions? ( } 0 \text { Never }-10 \\
\text { Always) }\end{array}$} & Implicit & $8(6-8)$ \\
\hline & Explicit & $8(7-9)$ \\
\hline \multirow{2}{*}{$\begin{array}{l}\text { Did the allocated intervention suit the participant? }(0 \\
\text { Very Poor }-10 \text { Very Good) }\end{array}$} & Implicit & $7(6-8)$ \\
\hline & Explicit & $7(6-8)$ \\
\hline \multirow[t]{2}{*}{7.} & Implicit & $7(6-8)$ \\
\hline & Explicit & $7(6-8)$ \\
\hline \multicolumn{3}{|l|}{$\begin{array}{l}\text { 8. (0 No change }-10 \text { completely changed i.e. } \\
\text { improved })\end{array}$} \\
\hline \multirow{2}{*}{$\begin{array}{l}\text { In your opinion, did the participant has the tendency } \\
\text { to develop explicit rules? ( } 0 \text { Not at all }-10 \text { to a great } \\
\text { extent) }\end{array}$} & Implicit & $6(3-8)$ \\
\hline & Explicit & $7(5-8)$ \\
\hline
\end{tabular}

Legend: IQR: Interquartile Range: 25-75 percentile 


\section{Tendency to develop explicit rules}

Related to the development of explicit rules (question 8), therapists mentioned that often the participants wanted to be in control (think about) of their own walking performance. Therapists described "as a person she wants to control" (explicit intervention, therapist 4) or "He thinks a lot about 'why' and 'what" is going wrong during walking' (implicit intervention, therapist 2). Additionally, therapists described that participants tended to use explicit rules that they may have acquired through care givers and through previous therapy sessions. For example, they mentioned that participants had a tendency to develop explicit rules for different reasons, such as "I think due to the classic (explicit) way of learning/improving gait" (implicit intervention, therapist 3), 'especially due to instructions that have been used in earlier phases of rehabilitation' (explicit intervention, therapist 1) and 'A lot was made explicit by the former therapists, partner and/or daughter' (implicit intervention, therapist 3). In response to future use of the learned instructions (question 4), therapists thought that some participants who received the implicit intervention would probably switch back to the use of explicit rules.

\section{Observed changes}

A variety of observed changes after the intervention were reported by the therapists ranging from improvements in "spatiotemporal parameters" (e.g. better foot clearance, faster walking, or a better step through gait) to changes in "cognitions and emotions" (e.g. more confidence in walking, ease of walking and more joy in walking).

\section{Patient experiences with regard to feasibility}

Table 6 represents the answers on participant questions related to their experiences with the received intervention. The participants were overall satisfied about the intervention and found the received instructions were relatively easy to use.

Table 5. Responses of the patient questionnaire on the intervention's feasibility

\begin{tabular}{|c|c|c|}
\hline End of 9 training sessions & Intervention & Median (IQR) \\
\hline \multirow{2}{*}{$\begin{array}{l}\text { How difficult or easy were the instructions of the training } \\
\text { sessions? ( } 0 \text { very difficult }-10 \text { very easy) }\end{array}$} & Implicit & $7(5-8)$ \\
\hline & Explicit & $6(5-8)$ \\
\hline $\begin{array}{l}\text { Did you gain more confidence in walking? } \\
\text { ( } 0 \text { Not at all }-10 \text { Very much) }\end{array}$ & Implicit & $7(6-8)$ \\
\hline & Explicit & $7(6-8)$ \\
\hline \multirow{2}{*}{$\begin{array}{l}\text { How did you experience the gait training sessions in } \\
\text { general? ( } 0 \text { Not satisfied }-10 \text { Very satisfied) }\end{array}$} & Implicit & $8(7-9)$ \\
\hline & Explicit & $8(8-9)$ \\
\hline
\end{tabular}

Legend: IQR: Interquartile Range 


\section{DISCUSSION}

The aim of this process evaluation was to assess whether the implicit and explicit motor learning walking interventions for people after stroke delivered in a randomized controlled trial were performed as intended (fidelity) and to report the therapists' and participants' experiences with regard to feasibility. Overall, the interventions have been delivered as intended with regard to fidelity and therapist and participants were generally positive about the feasibility of both interventions.

\section{Methodological quality, strength and limitations of the study}

Within the process evaluation three factors were observed that may have influenced contrast between the implicit and explicit interventions. First, therapists were exposed to knowledge of both interventions, and needed to constantly switch between interventions, which may have led to contamination. Providing both interventions may also be seen as a strength of the study as in real life therapists equally need to switch between different learning approaches. Second, therapist observed that overall participants had the tendency to self-develop explicit rules. They mentioned that some participants tried to consciously control their movements, which is a common characteristic of people after stroke.111,120 Furthermore, therapists reported that explicit rules may also have been acquired through support of caregivers and earlier received therapy sessions (prior to the study). The presence and possible use of explicit rules, may have caused the implicit intervention to lean more towards the middle of the implicit-explicit continuum. Third, therapists were more preserved in the provision of explicit rules in people with lower cognitive abilities which in turn this may also have contributed to a diminished the contrast between interventions. Despite good protocol adherence the process evaluation also showed that it is difficult to keep interventions $100 \%$ implicit or explicit. Incidentally (14\% of the implicit sessions; $1 \%$ of the explicit sessions) implicit instructions were provided within the explicit intervention and vice versa. Also, in some cases the number of explicit rules within the explicit interventions were very low.

A strength of the study was that the interventions and population reflect real life practice very well as the interventions took place in the home environment of the participants and implicit and explicit instructions were tailored towards the individual walking problem. Also, the identified factors that possibly influenced the interventions contrast, should be taken into account and cannot be ignored when translating findings to daily life practice. The high external validity, and positive experiences by therapists and participants contribute to the generalizability and transferability of findings to the overall stroke population and clinical practice. 


\section{Results compared to other studies}

To our knowledge this was the first study to examine the fidelity and experiences of an implicit and explicit motor learning walking intervention that took place in the home environment of people after stroke. This makes it difficult to compare results to other studies, however, some insights can be gained from studies in applied sports settings e.g.10,11. For example, with regard to contrast we observed a mean difference of five explicit rules between the implicit and explicit intervention. This is less than the difference of eight explicit and twelve explicit rules that were used within a basketball and respectively table tennis task.10,11 However, we don't know how many rules are needed to achieve a sufficient contrast between interventions.

Although comparable studies on motor learning are lacking, there are other process evaluations with neutral study results, that evaluated the implementation of complex interventions within clinical trials.147,148 These studies revealed that interventions were less feasible than expected.147,148 In contrast to those studies, the interventions within this trial were overall implemented as intended. Even when complex interventions in pragmatic trials are being carried out well, a variety of factors such as individuality (e.g. participants, therapists, caregivers), training environments, or context are difficult to control for.

Therapists not only observed changes related to walking speed (main outcome parameter of the trial) but also reported changes in other spatiotemporal gait, feelings of joy and confidence. Similar findings were observed in another process evaluation that evaluated a movement imagery intervention in people after stroke.147 Next to the primary outcome a variety of potential benefits e.g. related to confidence, were reported by the therapists. Future studies may consider incorporating different outcome measures that cover a broader range of improvements.

\section{Implications for research and clinical practice}

From the process evaluation various implications for research and clinical practice can be learned. Firstly, therapists experienced that partners or family members, with good intensions used explicit rules (thus indirectly violated the protocol) to explain how an analogy should be performed. Therefore, it is important to have similar views and standardize treatments (i.e. mutual understanding of implicit learning) with caregivers and members of the multidisciplinary team who treat the patient.

Furthermore, finding a suitable (meaningful) analogy may require time and the process of developing a successful analogy may take longer than one therapy sessions. It can be a creative process as the analogy should reflect correct movement pattern and preferably is meaningful to the participant.24 Examples of analogies to facilitate walking are reported (table 3), also a few other analogies to facilitate gait have been described by 
Kleynen et al24 and Jie et al.68 We hope that sharing the delivered analogies (table 4) may function as a source of inspiration.

Tailored implicit and explicit motor learning walking interventions can be applied in a feasible manner to people after stroke who are in the chronic phase of recovery. However, various factors such as cognitive (in)abilities or the tendency to self-develop explicit rules can influence the implicit or explicit nature of the intervention.

\section{Conclusion}

The delivery of the implicit and explicit motor learning walking interventions were successful in terms of fidelity. Within the process evaluation three factors were observed that may have influenced contrast between the implicit and explicit interventions e.g. less explicit rules were provided in people with lower cognitive abilities. Therapists and participants were generally positive about the intervention's feasibility.

\section{Acknowledgements}

This work was supported by Nationaal Regieorgaan Praktijkgericht Onderzoek SIA (RAAKPRO; grant number 2014-01-49PRO). The authors would like to thank the therapist and patients who contributed in conducting the process evaluation within this trial 



\section{CHAPTER 7}

\section{Design of the user interface for "Stappy", a sensor-feedback system to facilitate walking in people after stroke: a user-centred approach}

Li-Juan Jie, Gaston Jamin, Kate Smit, Anna JHM Beurskens, Susy M Braun

\section{Published as:}

Jie, L. J., Jamin, G., Smit, K., Beurskens, A., \& Braun, S. (2019). Design of the user interface for "Stappy", a sensor-feedback system to facilitate walking in people after stroke: a user-centred approach. Disability and Rehabilitation: Assistive Technology, 0(0), 1-9. doi: 10.1080/17483107.2019.1629654

(C) 2019 Informa UK Limited, trading as Taylor \& Francis Group 


\begin{abstract}
Introduction: Sensor-feedback systems can be used to support people after stroke during independent practice of gait. The main aim of the study was to describe the user-centred approach to (re)design the user interface of the sensor feedback system "Stappy" for people after stroke, and share the deliverables and key observations from this process.

Methods: The user-centred approach was structured around four phases (the discovery, definition, development and delivery phase) which were fundamental to the design process. Fifteen participants with cognitive and/or physical limitations participated (10 women, $2 / 3$ older than 65 ). Prototypes were evaluated in multiple test rounds, consisting of 2-7 individual test sessions.
\end{abstract}

Results: Seven deliverables were created: a list of design requirements, a personae, a user flow, a low-, medium- and high-fidelity prototype and the character "Stappy". The first six deliverables were necessary tools to design the user interface, whereas the character was a solution resulting from this design process. Key observations related to "readability and contrast of visual information", "understanding and remembering information", "physical limitations" were confirmed by and "empathy" was additionally derived from the design process.

Conclusions: The study offers a structured methodology resulting in deliverables and key observations, which can be used to (re)design meaningful user interfaces for people after stroke. Additionally, the study provides a technique that may promote "empathy" through the creation of the character Stappy. The description may provide guidance for health care professionals, researchers or designers in future user interface design projects in which existing products are redesigned for people after stroke.

\title{
IMPLICATIONS FOR REHABILITATION
}

- The case study provides a structured methodology and seven deliverables that may contribute to the (re)design of interfaces of existing supportive technologies for stroke rehabilitation.

- For supportive technologies in stroke rehabilitation important aspects to consider are the provision or presence of "feedback" (sensor-feedback system), "readability and contrast of visual information", "understanding and remembering information", "physical limitations" and "empathy".

- Apart from functional requirements and an understandable user interface, i.e., good usability, our case study demonstrates that the inclusion of a (fictional) character like "Stappy" may lead to a more meaningful and enjoyable user experience.

98 | Design of the user interface for "Stappy" 


\section{INTRODUCTION}

The number of people affected by stroke continues to rise throughout the world due to an ageing population.149 About two-thirds of stroke survivors experience difficulties in walking.101 It is known that frequency and intensity within a functional approach are essential prerequisites for effective rehabilitation.150,151 However, in the first stages after discharge from hospital or inpatient rehabilitation facilities, therapy time is often limited, whereas at the same time stroke survivors still feel they would benefit from more therapy.152 Effective and efficient therapy is essential to cope with the increased numbers of incidence and prevalence of stroke survivors.

To meet the required training intensity, which is needed for optimal recovery, patients are encouraged to practice independently at home. Unguided practice may not only contribute to physical recovery but may also improve feelings of autonomy. However, the actual compliance by patients at home to therapist-prescribed activities seems poor.32,153 The presence of cognitive impairments, which is commonly seen in people after stroke, 154 may be one of the explanations. People can forget to exercise (e.g., due to memory deficits), find it hard to monitor own performance (e.g., due to attentional deficits) or may not be motivated enough to practice. Affordable and simple solutions, such as smart devices could support people after stroke during unguided practice.

Smart devices with sensor-feedback have the potential to stimulate and motivate users to practice. Moreover, they allow practice without therapy supervision at convenient times in the preferred (home) environment of the patient. An example of an easy to use sensorfeedback system is the CuPiD-system. ${ }_{34}$ Casamassima et al34 developed this wearable gait training system (Figure 1) for people with Parkinson, which was later adapted by Ferrari et al.155 The functionalities of the technology also seem compatible for gait rehabilitation in people after stroke. However, next to functionalities, designing a meaningful user interface for this target population may optimize the user experience and therefore the actual use of the system. We therefore wanted to examine whether the CuPiD-system could be altered for use within this new target population. The current study describes a user centred approach that was used to (re)design the user interface of this existing sensorfeedback system for people after stroke.

As input for the (re)design process, the designers needed a thorough understanding of the needs and preferences of the new target population to ensure a meaningful and comprehensible user interface. Therefore, as a first step of the design process (so called "discovery phase"), three main characteristics of the stroke population were identified from literature31,94,154,156 and user interfaces of related projects (Quick Board,157 Med App, 158 Oefen App beroerte159). The characteristics include "the older population (65)", "cognitive" and "motor impairments". For the user interface, these characteristics imply 
that the design should consider aspects related to readability,156 reduced cognition (e.g., minimize task complexity) 154 and motor impairments.31,94 Also, preferably, traditional and intuitive colours are used, e.g., "green" (good) and "red" (wrong) to match the mental model of the target population.160,161 Based on the stroke specific characteristics and literature, design requirements for the sensor-feedback system were established (Table 1). Design requirements were discussed and confirmed with potential future users in informal conversations.

Table 1. List of user requirements

\begin{tabular}{|c|c|c|}
\hline $\begin{array}{l}\text { Stroke specific } \\
\text { characteristics }\end{array}$ & Design Requirements for user interface & References \\
\hline \multirow{6}{*}{$\begin{array}{l}\text { Older population: } \\
\text { Readability and contrast of } \\
\text { information }\end{array}$} & $\begin{array}{l}\text { Feedback = basis (general principle \& function of } \\
\text { sensor-feedback system) }\end{array}$ & \\
\hline & - $\quad$ Large font size & 156,161 \\
\hline & - Clear call to actiona in page; & 157 \\
\hline & - $\quad$ High contrast use of colours; & 161 \\
\hline & - $\quad$ No hidden information (fold-outs, scrolling); & 159 \\
\hline & - Use of relevant pictures to complement the text & 156 \\
\hline \multirow{3}{*}{$\begin{array}{l}\text { Cognitive impairments: } \\
\text { Understanding and } \\
\text { remembering information }\end{array}$} & - Intuitive use of colours & 157,160 \\
\hline & $\begin{array}{l}\text { - Use of simple language (no jargon) and simple } \\
\text { instructions }\end{array}$ & $156,157,162,163$ \\
\hline & - $\quad$ Limited amount of text; & 161,163 \\
\hline \multirow{5}{*}{$\begin{array}{l}\text { Motor impairments: } \\
\text { Physical limitations }\end{array}$} & - Avoid complex interactions; & 162 \\
\hline & - $\quad$ No (fast) moving or animated elements; & 162 \\
\hline & - $\quad$ Relatively large buttons & $159,161-163$ \\
\hline & - $\quad$ Avoid holding a device during exercise; & $\begin{array}{l}\text { Clinical } \\
\text { expertise }\end{array}$ \\
\hline & - Easily secured sensors; & $\begin{array}{l}\text { Clinical } \\
\text { expertise }\end{array}$ \\
\hline
\end{tabular}

aCall to action (CTA): a key element on a webpage, acting as a signpost that lets the user know what to do next. 162163

The main characteristics and design requirements formed the departure point for the design process. In order to develop a meaningful and intuitive user interface, users were intensively involved throughout the entire design process. 
The current study was conducted to finetune and optimize the sensor-feedback system that is part of a larger usability study. The overall goal was to create a meaningful user experience for people after stroke through designing a usable and enjoyable interface of the sensor-feedback system. The second aim was to provide a systematic description of the user-centred approach with its associated deliverables. The identification of key observations may support the (re)design of existing and future products or user interfaces for the stroke population. The final result was a high-fidelity prototype of the sensorfeedback system, called "Stappy" (see Box 1 for description).

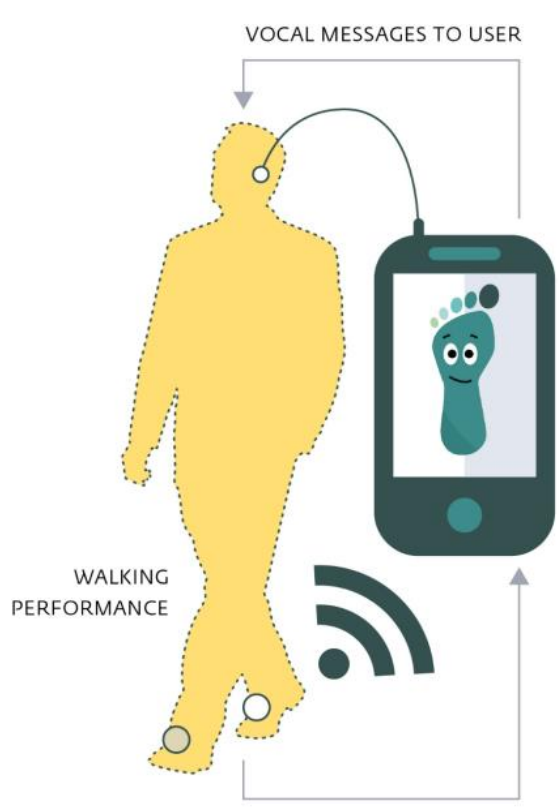

SENSORS PROVIDE REAL-TIME FEEDBACK
Box 1. About "Stappy"

- Stappy (Figure 1), is a spinoff of an existing sensor-feedback system originally developed for people with Parkinson's34.

- The system provides real-time feedback to users while walking.

- The overall concept and hardwaresoftware architecture of the system in described in detail by Casamassima et al34.

- The current study describes continued developments on the user interface of Stappy for the target population 'stroke'.

- The sensor-feedback system is named after the character Stappy, which was designed by Kate Smit. The character Stappy guides users through the steps in the smartphone application.

Figure 1. Overview of the sensor-feedback system, with sensors on the shoes, smartphone and auditory feedback through speakers. 


\section{METHOD}

The user-centred approach was structured around four phases, the discovery, definition, development and delivery phase (see top layer, Figure 2).164 The first (discovery) phase established the characteristics (translated into user requirements) of the target population. The discovery, definition, development and delivery phase are outlined below. Within each phase several deliverables were produced (see middle layer, Figure 2). To uncover as many issues as possible, small rounds of test sessions took place until all major usability problems were resolved. The duration of the entire design process took place over a oneyear period. This study is part of a larger usability study that was approved by the local ethics committee with reference number METC Z 17-T-06. All ethical principles, e.g., voluntary participation, privacy, confidentiality, were considered during the design process of the user interface.

\section{Participants}

Test rounds took place within the development phase in which participants evaluated the prototypes in individual test sessions. Participants were recruited from a local rehabilitation centre (Limburg, The Netherlands) and via client representatives of the project team. As cognitive impairments are a main characteristic of the target population (Table 1), for the first test round only people who experienced cognitive limitations in daily life were included. Furthermore, people were included in the test sessions if they were open towards use of technologies and had the goal to improve gait function. Each test round consisted of 2-7 individual test sessions. In total 15 participants (10 female and 5 male) of which 10 people with stroke took part in the study: the majority was older than 65 years (10/15) and all had cognitive and/or physical limitations. About half of the participants were familiar with smart devices, e.g., had previous smartphone or tablet experience. Demographic data of the included participants are presented in Table 2.

$\mathbf{1 0 2} \mid$ Design of the user interface for "Stappy" 


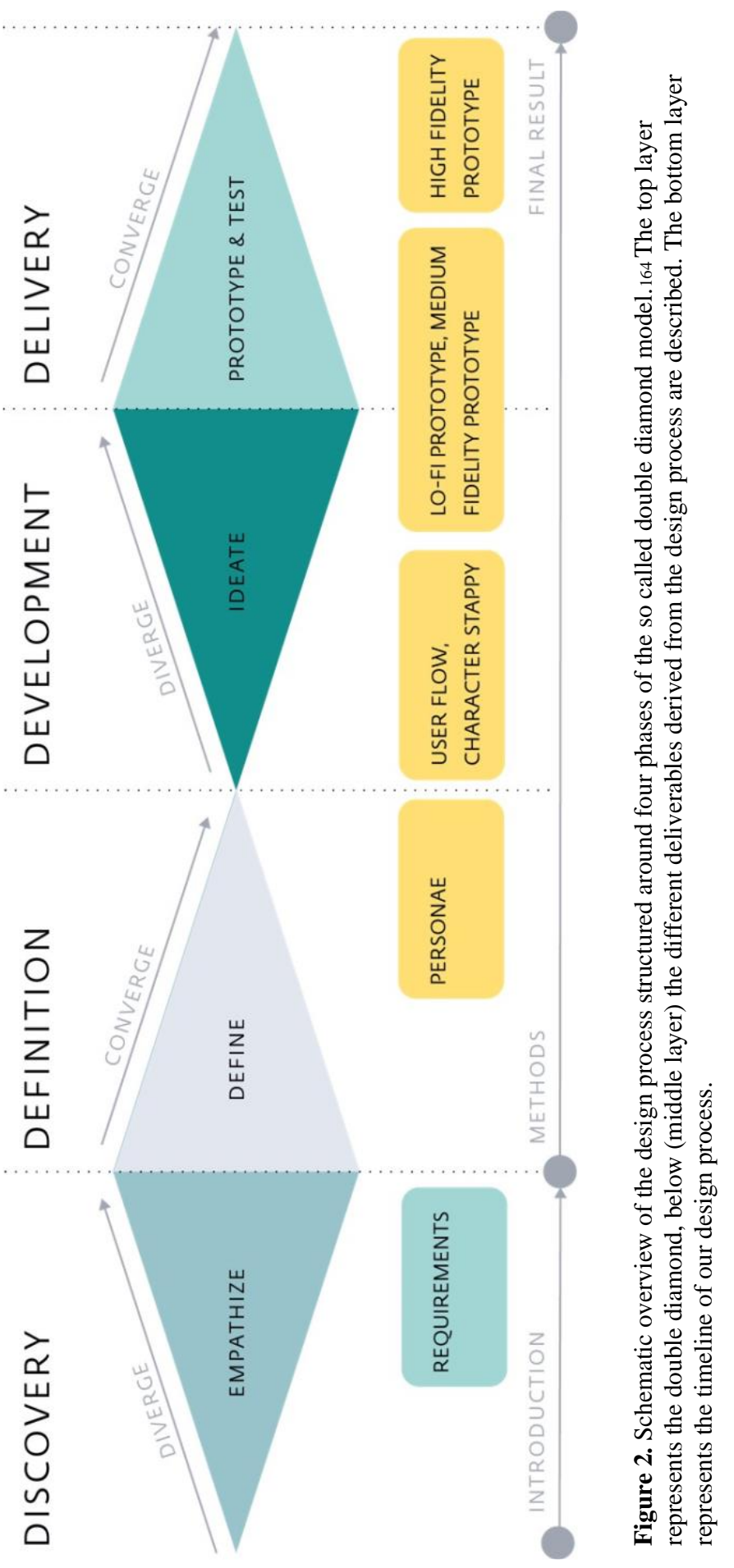




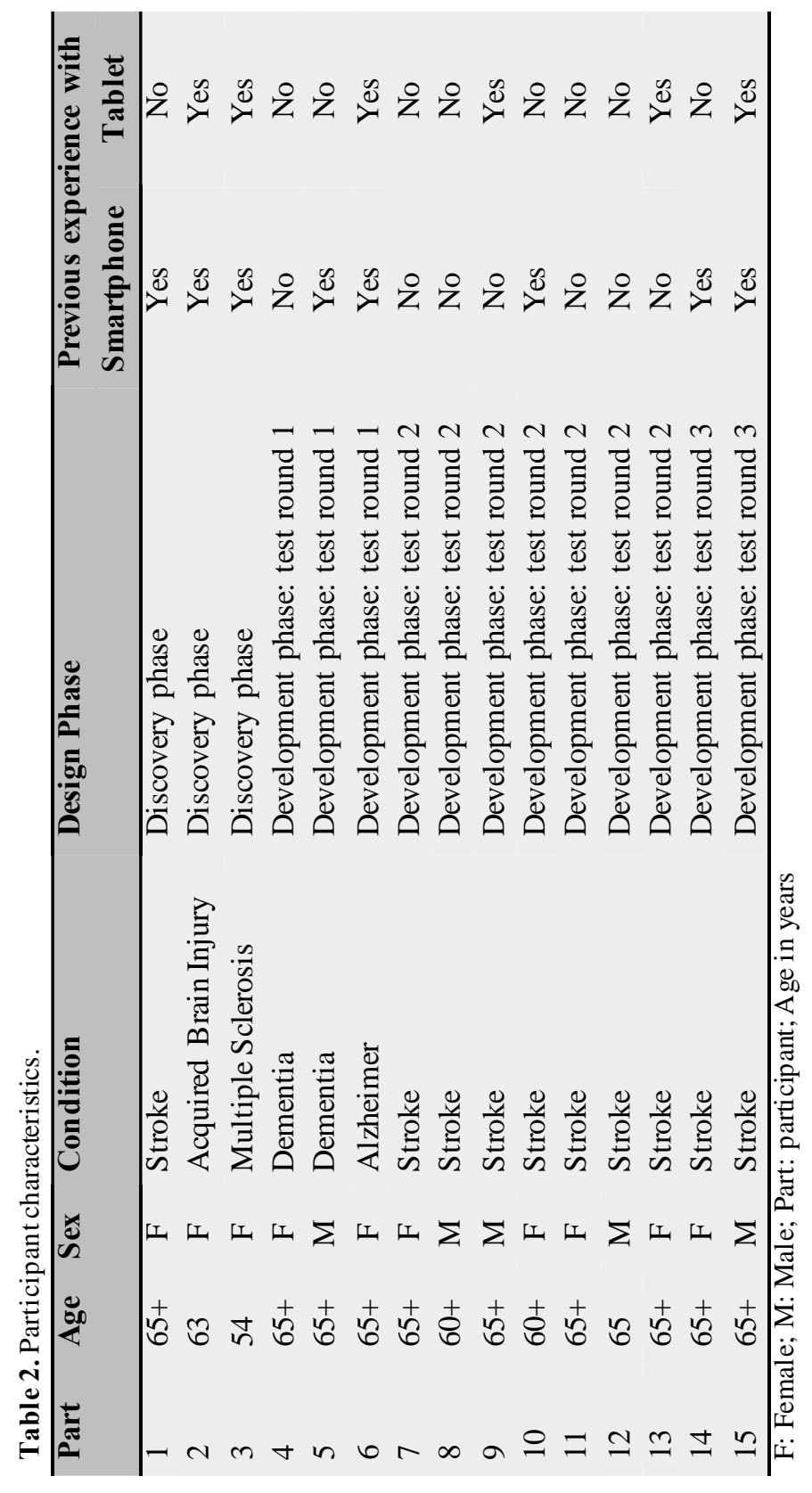




\section{Definition phase}

Based on literature and related projects (discovery phase), user requirements were gathered (Table 1). The list of user requirements was evaluated and if applicable extended during the design process, e.g., when new information derived from the test sessions. Knowledge derived from the discovery phase was synthesized into a persona. A persona can be defined as "an archetype of a user that is given a name and a face, and it is carefully described in terms of needs, goals and tasks" and is used by the design team to satisfy the user needs and goals. 165

\section{Procedure and analyses}

In the current study, the persona was based on the main characteristics and design requirements as identified from the discovery phase. This persona is a visual representation of the application's intended user.

\section{Development phase}

Test sessions with the deliverables, i.e., designed prototypes, began in the development phase. Based on the persona and design requirements (discovery and definition phase), the following deliverables were designed: a user flowa and low to high fidelity prototypes. In test sessions with potential future users, the user flow, low and medium fidelity prototypes were evaluated, the high-fidelity prototype is evaluated in the delivery phase.

\section{Procedure and analyses}

The test sessions involved the evaluation of different components. In the first test round, with regard to the user flow participants were asked to evaluate (1) whether steps were placed in a logical order, (2) whether text was clear and (3) whether provision of information was complete or what was missing. With regard to the prototypes they were asked to evaluate which they preferred (and why) and what they (dis)liked (and why). After test round one, all feedback was considered and processed in the design. In the consecutive test, round participants were asked to provide feedback on each component of the application. These included the homepage, the walking exercise and stopping the training. In test sessions, users reported what they (dis)liked about the following items: use of colours, readability, instructions, language and feedback. Test sessions took place until no major usability problems occurred. Key observations were summarized at the end of each test round. Examples of the designed prototypes along the design process were displayed in figures and clarifying quotes were reported.

\section{Delivery phase}

The delivery phase presents the results of the last round of test sessions, i.e., when no major usability issues were reported by the users. A high-fidelity prototype formed the starting point for the delivery phase. Test sessions were similar to the development phase, hereby users were asked to evaluate components related to use of colours, readability, 
instructions, language and feedback. Next to these components, if applicable, users could report other usability issues that were not listed. Together with engineers of the project team, the high-fidelity prototype "Stappy" was implemented in the existing smartphone application of the sensor-feedback system.

\section{RESULTS}

Within each phase, different deliverables (persona, list of user requirements, and prototypes) were created (Figure 2). In total three rounds of test sessions (iterations) were needed to develop a low, medium and high-fidelity prototype. The first two rounds are described in the development phase and evaluation of the high-fidelity prototype (third test round) is described in the delivery phase.

\section{Definition phase}

The definition phase resulted in a persona that is presented in Figure 3.

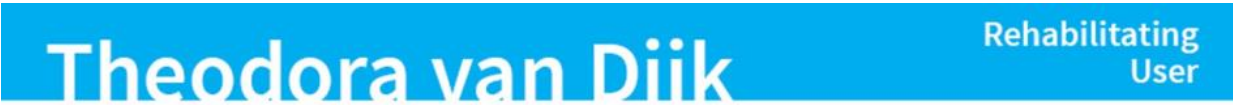

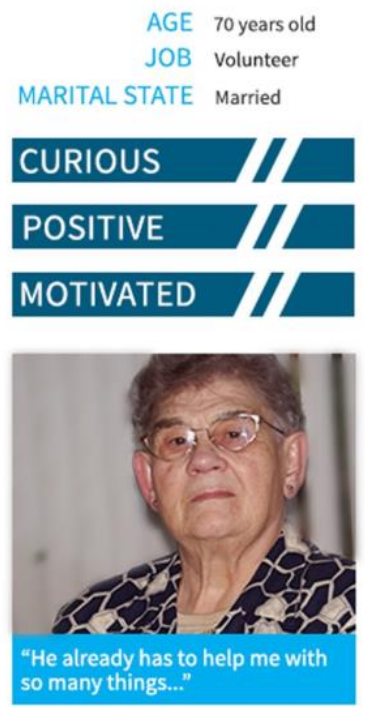

\section{USER NEEDS}

Would like to improve walking

(Re)building self-esteem

Likes to explore new things

Wants to be able to do things herself

\section{CHALLENGES}

Reading small fonts

Keeping balance during walk Energy level

Fine motor skills in left hand Assessing own training

To stay motivated when disappointed

\section{TECHNOLOGY}

Minimal experience smartphone

Minimal computer knowledge

Sometimes insecure in use of technology

Open to "give things a try"

\section{HER STORY}

Aged 69 Theodora had a stroke. She was alone when it happened. The moment she realised something was wrong, she called her son. Now, a year after her stroke, she is still working on her gait rehabilitation. She finds her husband to be of great support.

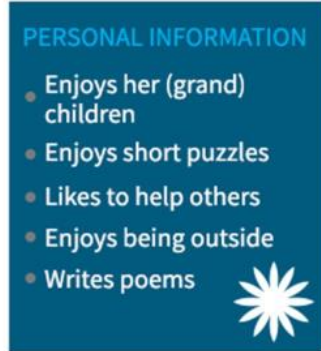

Figure 3. Persona of the application's intended user. 


\section{Development phase}

The development phase presents the prototype, key observations and evaluation by the users of each test round. Results of each test round are described as follows: first the deliverables are presented, then the evaluation of the deliverable are described and finally the key observations from the test round are summarized.

\section{Test round 1: user flow and low fidelity prototype}

The discovery phase delivered input for the design of the user flow (Figure 4) and low fidelity prototype (Figure 5). Three users evaluated the user flow, and low fidelity prototype of the user interface (Table 2). All three people understood and agreed with the user flow. They emphasized that the option to receive extra information if needed is nice. People were positive about the extra reminder at the end of practice to charge the system. Based on responses of the users in the first test round, no further changes were made in the user flow. All three people preferred the sketch that included a fictional character (Figure 5, right). People mentioned that the character made the design cheerful and personalized. Importantly, the character was not perceived as "childish" but users found the character suitable for the application. Users reported that the text "connect with the sensors" was not clear and reacted to the text with "but how do I do this?" (participants 4 and 5). The feedback from test round one and the remaining design requirements that could not yet be implemented in the low fidelity prototype (Table 2) were integrated in the medium fidelity prototype (Figure 6). Two versions (Figure 6) of the medium fidelity prototype with different colour options were evaluated in the following test round (Box 2).

\section{Box 2. Key observations: user flow and low fidelity prototype}

Known from literature and confirmed in study

- Understanding and remembering information: Cognitive limitations are common within the target population, therefore an extra reminder function to charge the sensors after practice was added in the user flow. Secondly, an optional step was included with extra instructions on how to use the sensorfeedback system.

- Readability and contrast of visual information: Based on the earlier defined user requirements, the low-fidelity prototype aimed for a clear and simple presentation of the functionalities and provision of information. To emphasize the user experience, part of the sketches in this prototype included a fictional character. 


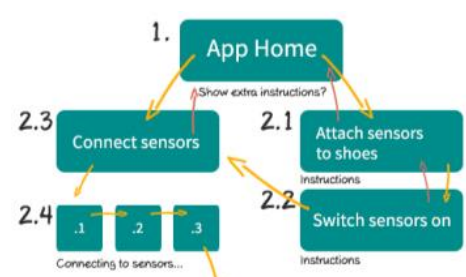

3.

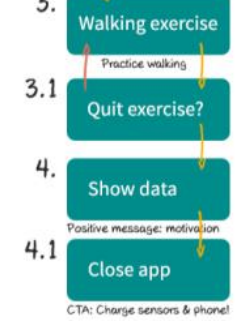

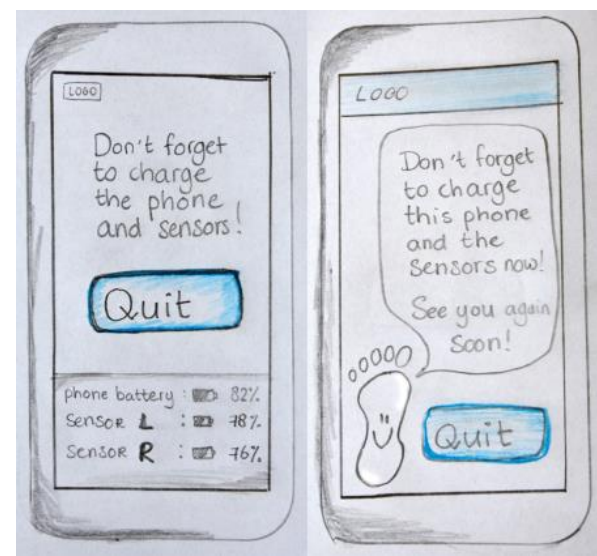

Figure 4. User flow of the mobile application. Figure 5. Low fidelity prototype

\section{Test round 2: medium fidelity prototype and character Stappy}

The first test round revealed that the user flow was clear, therefore, no other changes were made in the user flow. Furthermore, feedback from the low-fidelity prototype was processed in the design of the medium fidelity prototype (Figure 6) and the inclusion of a fictional character was further explored (Figure 7).
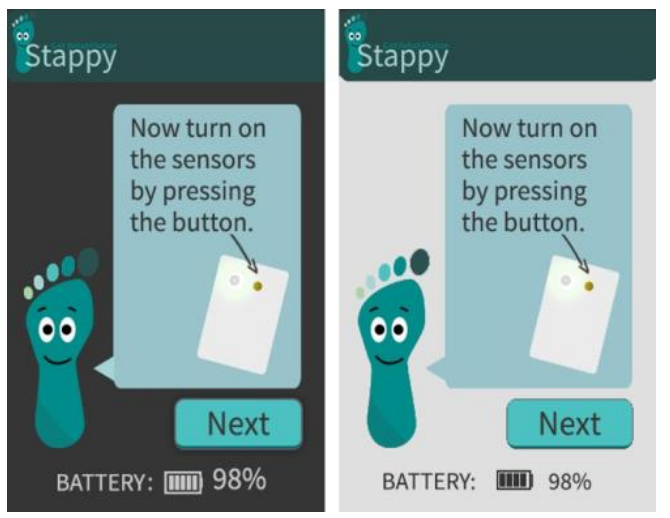

Figure 6. Medium fidelity prototype.

Version 1 (dark background); version 2 (light background).

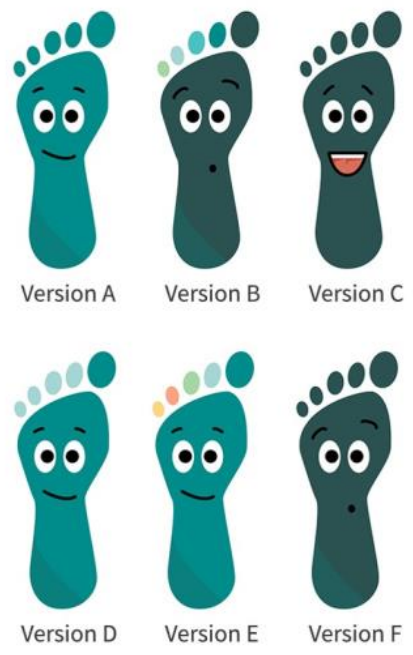

Figure 7. Exploration of the character "Stappy". 
In total, seven people evaluated the medium fidelity prototype of the user interface. People had a strong preference (six out of seven) for the version with a light background and letters displayed in black (Figure 6, left). Font and button sizes were found to be clear but some instructions were not. The screen with text "press to connect" (to turn on the sensors on shoes) brought some confusion. Text was intended to press on the button of the actual sensors but instead users pressed on a button of the user interface. For clarity some people proposed changes in the supporting image "the image should be more three-dimensional" (participant 9). Two users mentioned that it would be nice if the system would refer to their personal names. Users mentioned that they liked to receive feedback at the end of the training for example people reported "Even a low percentage would motivate me to do better next time" (participant 7) and "It would be motivational to have both the duration of practice as the success percentage as feedback" (participant 13). They reported that it would motivate them to keep on training. All seven people were positive about the character Stappy. Users stated "It's a funny foot, I like that it's there" (participant 13) and "Stappy makes it inviting to start training" (participant 10). The character was seen as a positive, enjoyable element of the interface that evoked sympathy "Stappy has a sympathetic look" (participant 9) (Box 3).

\section{Box 3. Key observations: medium fidelity prototype and character Stappy (test} round 2)

Known from literature and confirmed in study

- Readability and contrast of information: To optimize readability for the target population text was supported by illustrations.

NEW:

- Character Stappy: There was a strong preference for including a fictional character (test round one). Therefore, the addition of character Stappy was further explored (figure 7). Stappy was shaped as a foot so that people would associate character with the activity 'walking'.

- Empathy \& Feedback: The character Stappy can empathize with the users through displaying different emotions e.g. happy face when an action goes well. Through different emotions (empathy) the character Stappy also forwards some level of feedback. For example, when the user is waiting for the sensors to connect, the face expression of Stappy is waiting (figure 7b) until the connection is established. Then the character transforms into a happy emotion (figure 7A), connection succeeded. 


\section{Delivery phase}

With input from the development phase a high-fidelity prototype was designed. In the delivery phase, this prototype is evaluated. First, the high-fidelity prototype is presented, then the evaluation by the users and final key observations are described.

\section{Test round 3: high-fidelity prototype}

In the high-fidelity prototype, feedback from the second test round related to readability and the character Stappy were integrated (Figure 8). Two users evaluated the high-fidelity prototype and both mentioned that they were able to walk through the application easily. They reported to perceive the graphics as enjoyable "I like that Stappy's expression changes during the instructions" (participant 14) and "the instructions are clear and the images are a nice addition" (participant 15). The users confirmed that feedback at the end of practice and the built-in reminders were nice "I like the positive message about the score at the end of practice" (participant 14). Despite impaired motor skills (trembling hand) by one of the participants the buttons were big enough to "tap". One of the participants mentioned that she would like to see the battery status at the beginning of practice, while the other participant did not find this necessary. No other, major usability problems were experienced by the users and therefore this test round was determined as the end of the test sessions. Based on these results, no further changes were integrated in the user interface (Box 4).

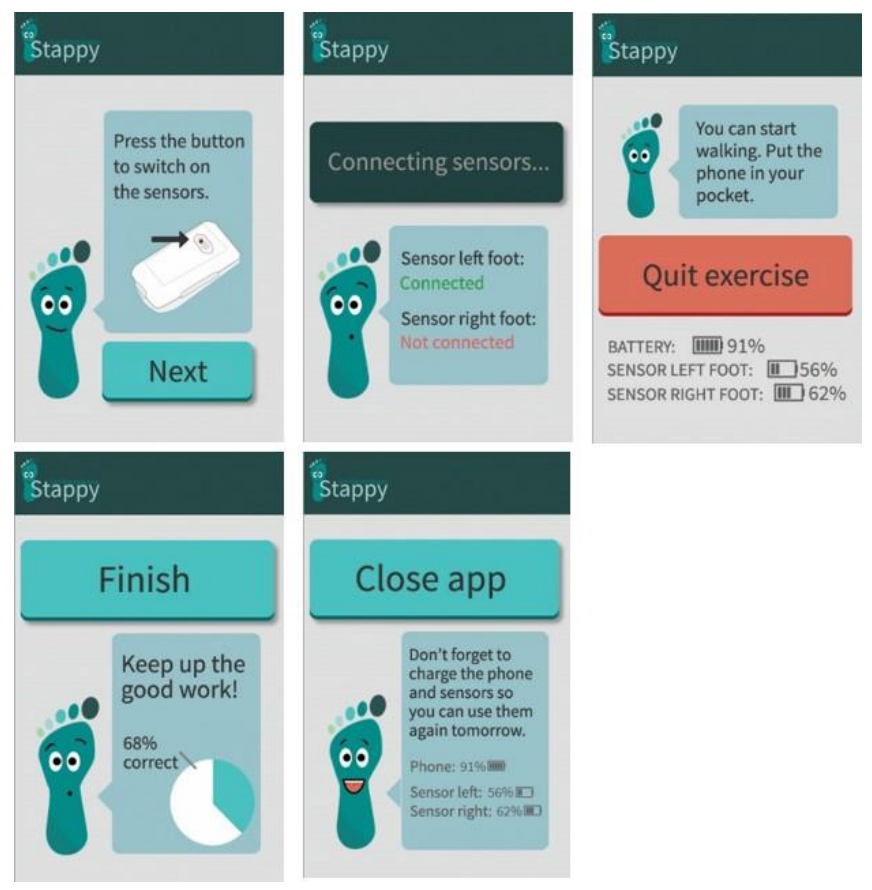

Figure 8. The final implemented user interface for the sensor-feedback system. 


\section{Box 4. Key observations: high-fidelity prototype}

Known from literature and confirmed in study

- Readability and contrast of information: The image of sensors was not clear. The graphic of the sensors was therefore transformed into a three-dimensional image and the associated text was adjusted to clarify that users should press the button of the sensors on the shoes (not the user interface itself).

- Readability and contrast of information: To keep provision of information to a minimum and avoid distractions from the actual task, battery status is only visible in the most relevant places (not on every slide).

- Feedback: Test round two pointed out the importance of feedback about performance. Therefore, at the end of practice the success rate of practice (percentage of correct performance) is shown in a pie-diagram.

\section{DISCUSSION}

The main aim of the study was to describe the user-centred approach used to (re)design a user interface from an existing sensor-feedback system for people after stroke, and share the key observations from this process. Within this approach, seven different deliverables derived: a list of design requirements, a persona, a user flow, a low-, medium- and a highfidelity prototype and the character Stappy. The iterative nature of the user-centred approach enabled us to gain a deeper level of user understanding and thereby design an interface that may lower the threshold for people after stroke to actually use the sensorfeedback system. Furthermore, the expressive personality of the designed character Stappy may contribute to a more enjoyable interaction with the product. Through the development of the interface, more general key observations could be obtained. Key observations covered aspects concerning "feedback" (sensor-feedback system), "readability and contrast of visual information" (older population), "understanding and remembering information" (cognition), "physical limitations" (motor impairments) and "empathy (character)". The first four listed domains of design requirements as reported in literature and observed in related projects (Table 1) were confirmed. "Empathy" was an additional domain derived from this specific design process. Feedback and empathy seem important motivational factors in general,166,167 and of course also for people after stroke.168,169 Hopefully, the description of the user-centred approach and associated key observations will contribute to the (re)design of existing and future products and interfaces for the stroke population.

\section{Comparison with other studies}

To improve designs of assistive technologies within the stroke population scientific literature advocates patient involvement throughout the entire design cycle.169,170 Comparable studies 170,171 that described the design process using a user-centred approach 
demonstrate similar design cycles. Starting with "getting to know" the users (e.g., through defining user requirements) after which products are developed and evaluated in an iterative manner with the users (e.g., through focus groups). This general design cycle could be placed in the double diamond model (see top layer, Figure 2).164 The double diamond model offers structure and was therefore used in the current study as a guidance to organize the design process. Below the double diamond in Figure 2, the deliverables created are presented, these may be different and specific for each design project. The choice of co-design techniques and deliverables may depend on various factors such as the expertise of the team, timeline, costs and main aim of the product.

Interface characters are a familiar concept within software technology 172; however, the design process of a character is not often explicitly described. Also, as far as we know, no other studies designing user interfaces for people after stroke have described and evaluated the application of an interface character like Stappy. Within the current study, the character Stappy seemed meaningful to users as it evoked feelings of sympathy and joy. Stappy was found to motivate people to practice, this is important as it may contribute to the overall goal of the system which is to optimize intensity and quality of independent practice of gait.

\section{Methodological quality}

There are some strengths and weaknesses in this study that need to be addressed. A strength of the study is that the design cycle as presented in Figure 2 does not describe a linear process and more- over there seems no golden recipe on how to proceed. The process requires flexibility and resilience of the design team. Following an iterative approach, structured around four phases (double diamond model),164 helped the design team to keep focus firmly on the user and make informed choices regarding methods and techniques. Truly understanding the user proved to be key in order to design a meaningful user experience.

A broad range of inclusion criteria was set in which cognitive limitations played a central role. Findings of the current study may therefore also be generalizable to other populations with similar characteristics for example older people and/or people that may experience cognitive limitations, e.g., people with Parkinson. Lastly, today there is a strong increase within current health care when it comes to the development of technologies.173 Sometimes there seems to be an overkill in similar products, instead of improving or altering existing ones. This study describes the changes made on a promising existing system 155 rather than focussing the developments of new technologies per population, which seems inefficient.

A weak point of the study might be the confirmation of the characteristics of the participants. The inclusion criteria stated that only people who experienced cognitive 
impairments could be included. Although all participants reported cognitive limitations in daily life, no cognitive tests were performed to confirm this. It is therefore not clear what range and severity of cognitive impairments in the current sample size was.

\section{Implications for research}

The (re)designed user interface should contribute to a positive and meaningful user experience. People should feel encouraged to practice their gait with the sensor-feedback system on a regular basis. Future research should assess the impact of the (re)designed user interface on the actual use sensor-feedback system. Furthermore, as the system is able to register frequency and duration of practice, it would be interesting to explore whether perceived usability can be linked to actual use of the sensory-feedback system. In other words, are people that evaluate the usability the highest, also the people that use the system the most (and vice versa).

Within this preliminary study, we observed that users were very positive about the potential use of Stappy. We believe that in some patients, the sensor-feedback system could really contribute to rehabilitation and unguided practice. In reality however, many technologies are not always used as regular as intended and the level of use is determined by various factors.174 To gain a better understanding of who to offer the technology (and who not) to, it would be interesting to explore which aspects motivate users the most to engage with the sensor-feedback system "Stappy" (compliance).

\section{Conclusions}

The study offers a structured methodology and seven deliverables with associated key observations that can be used for designing meaningful user interfaces for people after stroke. Furthermore, the study provides a technique that may promote "empathy" through the creation of the character Stappy. The description will hopefully provide guidance to health care professionals, researchers or designers that (are planning to) design meaningful and intuitive user interfaces for this target population. Future studies are needed to assess the actual impact of the (re)designed interface of this study on the usability of sensorfeedback system "Stappy" on walking performance of the users.

\section{Note}

a. The user flow is a requirement for designing the user interface of the application and depicts the path that a user follows through to complete a certain task, e.g., connecting the sensors to the phone.

\section{Acknowledgements}

The authors would like to thank the participants that took part in the study. We thank Elisabetta Farella and Bojan Milosevic for their openness and support in integrating the new user interface in the technology. 


\section{Disclosure statement}

The authors report no conflict of interest.

\section{Funding}

This study is financially supported by Nationaal Regieorgaan Praktijkgericht Onderzoek SIA (RAAKPRO; grant number 2014- 01-49PRO). 


\section{CHAPTER 8}

\section{General Discussion}


The overall aim of this $\mathrm{PhD}$ thesis was to develop and explore the application of different implicit motor learning approaches and to assess the effectiveness and feasibility of one implicit motor learning approach at home in people after stroke. This implicit approach was compared to an explicit motor learning approach. Additionally, the design of a user interface for a simple, innovative technology that can be used to support independent practice of walking at home, was developed.

Figure 1 presents the overall structure of the general discussion. First, the main findings of each phase within this thesis will be described. The first phase focused on exploring the application of different implicit motor learning approaches. Experiences and immediate effects of implicit motor learning within gait rehabilitation were described in chapters $2 \&$ 3. These studies provided insights that fed into the second phase of the thesis. In the second phase, a randomised controlled trial took place in which an implicit and explicit motor learning walking intervention for people after stroke were compared. From this phase, the protocol, results and an evaluation regarding the effectiveness and process of trial were described in chapters 4, 5 and 6. The last phase of this thesis described a methodology that was used to re-design the user-interface of an existing sensor-feedback system that supports people after stroke during independent practice of walking. The main findings are following by the methodological considerations, lessons learned and implications for practice, education and research.

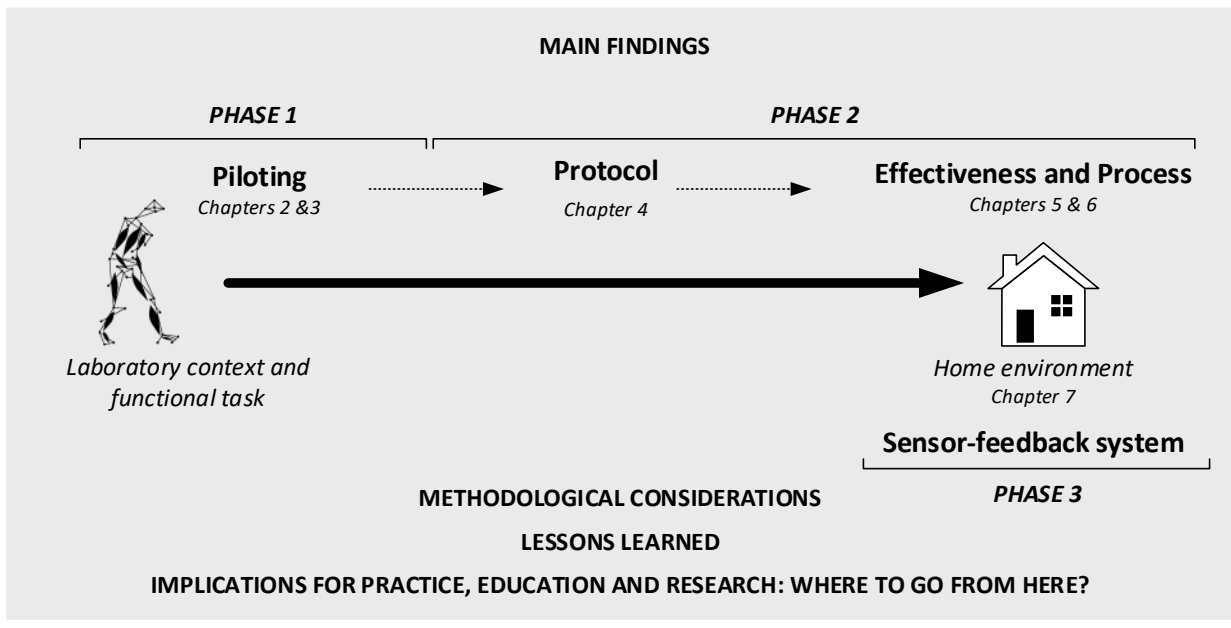

Figure 1. Overview of the structure of the general discussion 


\section{Main findings}

The main findings of this project are described within the three phases of the project.

\section{Phase 1: Exploring the application of different implicit motor learning approaches}

Implicit motor learning to facilitate walking performance seems feasible and is a potentially effective approach in people with Parkinson's and people after stroke. However, the effects may especially potent when a more personalised approach is used. In two pilot studies, we explored the application of three, potentially implicit, motor learning approaches. These were analogy learning, environmental constraints and action observation. In chapter 2, the analogy of "following the footprints in the sand" was used to enhance stride length in people with Parkinson's disease, thereby improving their walking performance. The analogy seemed meaningful to most of the participants, as the majority of people (10 out of 12) had a positive association with the beach. A positive trend towards robust dual-task performance was observed, supporting the potential implicit nature of the intervention. In chapter 3, we explored the immediate changes in gait following analogy instructions, environmental constraints and action observation in people after strokea. On a group level, only the analogy instructions and environmental constraints led to specific, immediate changes in walking performance. Within each strategy, the responses of the participants varied considerably. This was possible a consequence of the pre-defined, rather than tailored. instructions to the individual participants. The variety of personal associations with the instructions, either positive or negative, may have contributed to the wide variation in performance. The examined motor learning approaches seem feasible to facilitate walking performance in people with Parkinson's and people after stroke but may need to be tailored towards 1) the individual walking problem (e.g. biomechanical aspect) and 2) the individual preferences (e.g. experiences).

\section{Phase 2: The development, effects and evaluation of an implicit and explicit motor learning walking intervention}

Implicit (analogies) and explicit (detailed verbal instructions) motor learning walking interventions have similar effects on walking speed in people after stroke and can be personalised towards the individual walking problem and preferences of the participant. Chapter 4 describes how the tailored interventions can be applied to train walking performance in the home environments of people after stroke. To minimise the chance of spontaneous recovery, only people after stroke who were in the chronic phase of recovery were included in the study. In chapter 5 , a total 79 people after stroke were randomised to

\section{a Example cases}

Analogy Learning

"Walk as if you are following footprints in the sand"
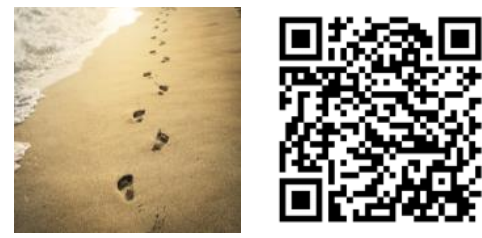

Environmental constraints

"Step only on the projected stripes while walking"

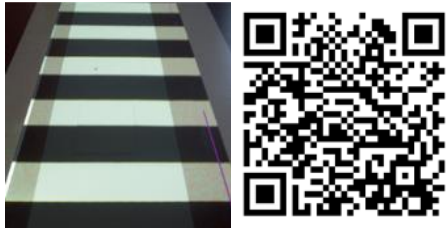


either an implicit or explicit motor learning walking intervention under guidance of a physiotherapist in their home environment. The implicit motor learning intervention had similar positive effects on walking speed compared to those who received an explicit motor learning intervention. No statistically nor clinically relevant differences were observed between groups. Over time, clinically relevant improvements for within group differences were observed in both groups. Chapter 6 revealed that the interventions had been delivered as intended and therapist and participants were generally positive about the feasibility of both interventions. Some factors were observed that may have influenced the contrast between the implicit and explicit interventions, such as the tendency to develop explicit rules and cognitive (in)abilities of the participants. Importantly, as the study demonstrated a high external validity, these findings seem highly generalizable and transferable to the overall stroke population and clinical practice.

\section{Phase 3: Designing a user interface of a gait sensor feedback system for use in stroke rehabilitation}

High-intensity and repetitive-task training are important factors for effective therapy.31 Therefore, also after discharge from an inpatient rehabilitation facility, where practice usually is very intensive, people are often encouraged to practice independently at home. However, generally therapy compliance is low, some people forget to practice, or the lack of motivation or feedback can be perceived as barriers to practice. 32 Supportive technologies with real-time feedback can be used to facilitate this process. Within this study (chapter 7), we choose not to develop something new, instead we tried to optimise an existing system (CuPiD system) 34 for people after stroke. In chapter 7 , in an attempt to increase the user experience and actual use of an existing sensor-feedback system, the user interface was re-designed specifically for, and together with, people after stroke.34 Apart from functional requirements and an understandable user interface, i.e., good usability, our case study demonstrated that the inclusion of a (fictional) character, like "Stappy", may lead to a more meaningful and enjoyable user experience.

\section{Methodological Considerations}

Within this section, the overall methodological considerations of the thesis will be discussed. The methodological considerations are described in relation to the following themes: "Order of chosen research designs", "From isolated tasks in laboratory settings to clinical practice", and "A structured process to design the user interface". 


\section{Order of chosen research designs}

A strength of this thesis is that research designs were carefully chosen based on the research question and stage of research, which fits well within the phases as described by the medical research council to develop and evaluate complex interventions.30 Through pilot studies,24,68,132 we first gained experience in the application of implicit motor learning to a functional task in neurological populations within a laboratory research environment. With a greater theoretical and practical understanding, we then set-up a larger clinical trial to examine the effectiveness (chapter 5) and process (chapter 6) of implicit motor learning within gait training in people after stroke in their home environments. We gained more insight into the effectiveness, fidelity and experiences of therapist and patients (chapters 5 to 6 ).

\section{From isolated tasks in laboratory settings to functional tasks in clinical practice}

To reflect real life practice, certain methodological choices with regard to the walking intervention and setting, selection of the participants, choice of the primary outcome measure, and operationalization of the interventions were made.

First, to our knowledge this was the first study to examine the effectiveness of an implicit motor learning walking intervention in people after stroke, that included a functional task (walking) and took place within the home environments of the participants.

Second, with regard to the selection of the participants, overall we included a heterogeneous sample of stroke patients with regard to different characteristics such as the physical and cognitive abilities. We only included people who were in the chronic phase of recovery to minimise potential improvements due to spontaneous recovery. A strength of this heterogeneity is that the population reflects real life practice very well. However, the heterogeneous population can also be viewed as weakness of our study as this may decrease the power of the study. From a theoretical perspective, most benefits of implicit motor learning may be expected for people with lower cognitive abilities. However, this specific target population was small $(n=15)$ within our heterogeneous sample, therefore no statistical sub-group analysis on cognition was performed. Furthermore, a weakness with regard to selection of the participants, is the erroneous inclusion of ten participants whose walking speed was too fast at baseline which may have led to a ceiling effect and consequently reduced power.

Third, we choose the 10MWT as the primary outcome measure. A strength of the 10MWT test is its good methodological properties138 and its common use within neurological rehabilitation which makes it easy to compare findings to other studies.134 Changes in walking speed have been associated with integrated results on other gait parameters such as step length and frequency 133,134 and also with changes in functional scales and abilities in daily life.138,175 The outcome may also be seen as a weakness. One could question whether the 10MWT is sensitive enough to detect differences if such underlying changes are small or not necessarily related to walking speed (e.g. improvements of confidence during walking).

Fourth, in order to keep the interventions feasible for clinical practice specific choices were made with regard to the operationalization of the implicit intervention that was structured by analogy learning. Rather than using fixed instructions (e.g. as done in Liao et al11 or Lam et al10), we 
personalised the analogies towards the individual needs and preferences. A strength of the personalised approach is that it allows flexibility to meet the participants needs (e.g. the individual walking problem, and physical and cognitive abilities of the participant) and contributes to the external validity of the study.

A weakness with research within a representative sample, real-world environment, and functional task is that various factors may have influenced the contrast between interventions (see table 1). However, many of these factors cannot be controlled for or it may not be desirable to control for in pragmatic trials that should represent for clinical practice.176 From a research perspective, it is important to keep to the extremes of the implicit-explicit continuum while for clinical practice it seems difficult to keep the interventions $100 \%$ implicit or explicit.

Table 1. Overview of observed factors that may have influenced the contrast between the implicit and explicit intervention in daily practice.

Implicit Condition

Explicit rules received by caregivers or partners

\section{Explicit Condition}

Therapists used less explicit rules in people with lower cognitive abilities

Participants tendency to selfdevelop explicit rules

Explicit rules acquired through former therapy

Therapists used less explicit rules in people with aphasia 


\section{A structured process to design a user interface}

To re-design the user-interface of the sensor feedback system, we used the double diamond model as described by the British design council to structure the design process.164 A strength of this model is that it provides the space and freedom to select the design techniques (e.g. creating a persona, creating a customer journey map or user-flow) that are most appropriate for each unique design project. Although the model allows flexibility it also provides structure through the presentation of four structured design phases: the discovery, definition, development and delivery phase.

A weakness of this thesis is, that only the design process of the user-interface has been described within this thesis. Any pilot tests or evaluations that further explored the actual use and experiences of patients and therapists with the device are lacking, as it was not feasible within the time frame of this thesis. However, part of these results are described here $177-180$ and other results will later be described elsewhere.

\section{Lessons Learned}

The lessons learned from this PhD-trajectory can be clustered in seven major themes: "The difficulty to determine effectiveness of implicit motor learning in clinical practice", "Personalised and meaningful analogies", "Tailoring motor learning: the importance of identifying participant characteristics", "The need to understand implicit motor learning within neurological rehabilitation", "Empathy through the creation of the character Stappy" and the "The value of patient participation".

\section{The difficulty to determine effectiveness of implicit motor learning in clinical practice}

Despite the well-considered outcome measures, the carefully designed interventions, and experienced therapists, we did not find any meaningful differences between the implicit and explicit motor learning intervention (chapter 4). It may be that there is no difference between the groups or we may not have been able to detect any differences between groups. From this specific trial we learned that when it comes to implicit motor learning there are various factors that may influence the intervention (see table 1).

Not only in our trial, but also other Randomised Controlled Trials (RCTs) have shown that it can be difficult to examine the effectiveness of motor learning approaches within clinical settings, heterogeneous samples and functional tasks.147,148 RCTs are generally seen as the gold standard to determine effectiveness of an intervention.181 However, there are challenges when applying RCT in its cleanest form within a clinical setting and with a complex intervention. In pragmatic trials with complex interventions, effects are not only dependent on the intervention, but are also strongly linked to the context.182 In order to reflect real-life practice (increasing external validity), often compromises are made that affect the internal validity of the study. RCTs are widely viewed on a continuum from explanatory (ideal situation) to pragmatic (usual care).183,184 Especially when close to the pragmatic end of the continuum, researchers should carefully weigh the pros and cons related to transferability of results (external validity) versus the ability to detect effects due to the intervention. To gain insights into the effects of an intervention, other research designs could be considered such as a multiple baseline design, or step wedge design. However, each design comes with its own strengths (e.g. opportunity to measure possible effects of the time) and limitations (e.g. 
time consuming, or reduced internal validity in comparison to explanatory RCTs). The research question should be the leading factor in the choice of design but the context should also be carefully considered. Pragmatic trials may not be the right approach if there is too much 'noise' due to the high external validity of the study.

\section{Personalised and meaningful analogies}

Analogies need to be personalised towards the individual walking problem and personal background and experiences of the learner. Taking in account the background of the learners may help to develop an analogy that is more meaningful to the learner. The importance of developing meaningful analogies was also reported in other studies.24,85 Poolton and colleagues 85 found that an existing and validated analogy effective within native English speakers did not work for a Chinese population. Therefore, they re-developed an analogy that was culturally relevant and thus, more meaningful for the Chinese learners which led to performance improvements as expected.85 An effective analogy for one person may not necessarily work for another person. It is important to keep the analogy simple and the complexity of the presented concept should to high.15 Generally, meaningful improvements can be observed relatively shortly after introducing a suitable analogy.24 If the patient doesn't understand the analogy, or if the analogy needs to be explained, this is not the right instruction.

Up until now, the use of personalised analogies as conducted within our trial (chapters 3 to 5) is different from the majority of studies on analogy learning that use more rigid protocols with fixed instructions e.g.10,11,132. However, we learned that it is feasible and effective to use personalised analogies to facilitate walking performance in people after stroke. Eventually, in order to reflect complex daily life situations, instructions should be tailored towards the individual needs and preferences of the learner.

\section{Tailoring motor learning: the importance of identifying participant characteristics}

In order for therapists to tailor motor learning towards the individual patient it is important to uncover which patients benefit most from which (implicit or explicit) motor learning approach. In chapter 3, we observed that especially people with better physical function (i.e. faster walking speed at baseline, higher scores on the Motricity Index, Brunnstrom Fugl-Meyer assessment and Berg Balance Scale compared to that of the rest of the group) responded best to the analogy instructions and environmental constraints. Recently, also other factors in people after stroke related to motor skills, cognition and attention, sensory function, and inclination for conscious control have been described as potential participant characteristics that influence motor learning (for an overview see185). For example, in a recent randomised trial in people after stroke, Kal et als6 observed that external focus (more implicit) instructions led to greater performance improvements in a balance task, with better balance and better sensory functioning, while internal focus (more explicit) instructions seemed more beneficial for those with more severe impairments.86 Also in other populations (e.g. in children or healthy adults) specific participant characteristics such as motor learning preference 186 and working memory 187 have been identified in relation to motor learning approaches. More research is needed to validate and identify patient characteristics and associated motor learning approaches in a variety of functional tasks within neurological rehabilitation. 


\section{The need to understand implicit motor learning within neurological rehabilitation}

Despite the well-considered measures to assess implicit motor learning, no clear picture could be obtained regarding the implicit nature of the intervention. One of the main hypotheses that is typically assessed within implicit motor learning is whether this approach is less cognitively (working memory) demanding compared to explicit motor learning e.g. see10-12. In chapter 2, we found some trends towards accepting this hypothesis, however we were not able to confirm this finding within the trial. Dual tasking is a commonly used measure to gain more insight into the attentional demands after learning a motor task. Within the trial, high variability in terms of cognitive abilities led to the inability to legitimately interpret the dual task results. This hypothesis is an important rationale for researching implicit motor learning within the neurological population. Also, knowing whether this hypothesis is true, may help practitioners to make well-informed decisions regarding the treatment approach. More research is needed to determine whether the potential mechanisms of implicit motor learning also apply for functional tasks within the neurological population.

\section{Empathy through the creation of the character Stappy}

Next to more general design requirements such as the importance of readability 156 or feedback, 169 an additional domain 'empathy' was identified within the design process of Stappy (chapter 7). The character Stappy (shaped as little foot, which refers to walking; see figure 1) takes the user through the different steps in the app. Through displaying different emotions (empathy) e.g. a happy face when an action goes well, the character also provides some level of feedback. Users reported that Stappy evoked feelings of sympathy and joy. Next to a functional user interface, future designers may create characters such as Stappy within the user-interface design to create a meaningful and enjoyable user experience for the target population, which in turn may optimise the actual use of the system.

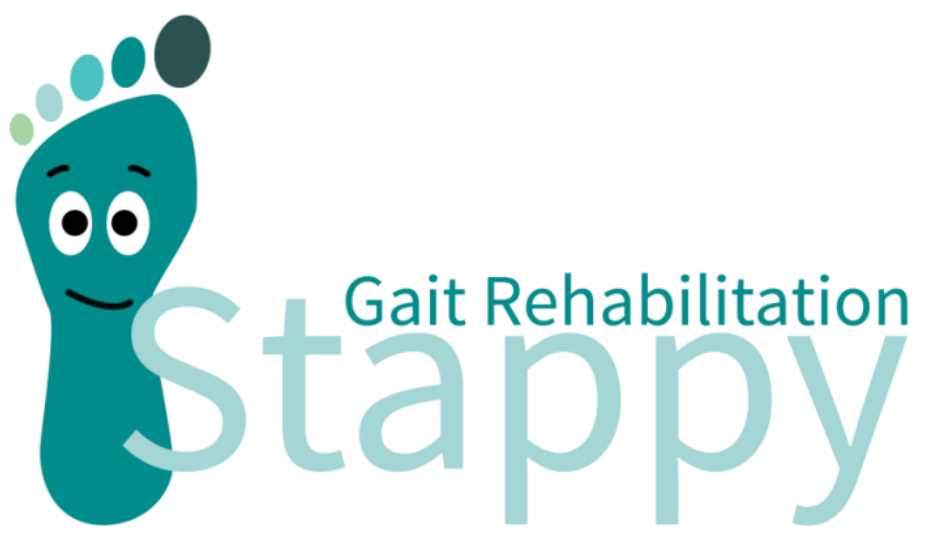

Figure 1. The character Stappy 


\section{The value of patient participation}

The involvement of patient representatives within this project was on a wide variety of levels 188 . From only receiving information about the project, to providing advice on the studies, and even up to being in charge e.g. the representatives published an article about the value of patient participation. The patient representatives helped us to keep the focus firmly on the target population and helped us to make informed decisions on aspects related to the applied methodologies, recruitment strategies, and dissemination of findings. Together with the patient representatives, various materials were developed, for example to facilitate communication with the participants, to preserve the feasibility of the measurements and training procedures, or to disseminate findings, which resulted in a more meaningful and more feasible project. We were very fortunate to have worked with individuals with specific expertise derived from being a patient, who were trained to actively participate in research projects, and had backgrounds in physiotherapy and other related health care areas.

\section{Implications for practice, education and research: Where to go from here?}

The following section will describe the implications for practice, education and research on implicit motor learning within neurological rehabilitation and which steps should be explored next.

\section{Implications for practice}

In 2015, evidence on implicit motor learning within neurological rehabilitation was limited and the available evidence was often not related to common daily life tasks such as walking. Knowledge transfer towards clinical practice can be achieved through education of (future) health care professionals. For clinical practice it especially important to publish research protocols with a detailed description of the interventions e.g.135,189,190, frameworks or practical protocols that describe how interventions can be applied e.g.191-193 and process evaluations e.g.147,148. Such papers may offer tools, examples and inspiration for therapists to structure their therapy sessions. Also, the current thesis describes relevant information about the application of implicit motor learning within the neurological population.

The developed implicit motor learning interventions within this thesis are concrete examples and may serve as a source of inspiration for (future) therapists. Especially, with regard to analogy learning a variety of analogy instructions to facilitate walking performance have been reported (see chapters 2, $3 \& 6$ ). The reported analogies can be used as a starting point and as inspiration, which can be especially helpful when experience with analogy learning is minimal. Furthermore, we have also described that personalised analogies can be developed. This thesis demonstrated that motor learning approaches can be tailored towards the individual needs, preferences and abilities of the patients in a feasible manner. The detailed description of the interventions in chapter 4 , and the developed intervention guideline (only available in Dutch) can be used by (future) therapists as a guide to structure therapy in either a (more) implicit or (more) explicit manner.

Furthermore, the process evaluation also revealed the importance of communication. It is important to communicate about the treatment plan with other people who treat the patient. For efficient therapy and to avoid confusion for the patient, make sure that everyone (i.e. partners, care-givers or 
other members of the multidisciplinary team) who treat the patient have similar views on the treatment approach. In this way, the learner will not be exposed to explicit rules while the aim is to provide therapy in an implicit manner and vice versa. Within a multi-disciplinary team setting, frameworks as developed by Kleynen et al 192 could potentially be used (e.g. added to the patient dossier) to structure this process. When it comes to clinical practice, the process evaluation also revealed that we can come very close the implicit extreme of the implicit-explicit continuum but that it seems difficult to keep the interventions a $100 \%$ implicit or $100 \%$ explicit.

\section{Implications for education}

In order to facilitate the knowledge transfer towards education, it important to create material and learning environments that are understandable and inviting to use for students and health care professionals. Rather than frontal teaching, interactive teaching methods and active use of multimedia materials may be more enjoyable, fun and engaging. Within the project a variety of materials have been developed such as short video clips about implicit motor learning, a video with therapists' experiences on implicit motor learning in people after stroke, example videos of participants walking within constraint led approach and analogy learning conditions, an instruction manual of the sensor-feedback system for people after stroke (also usable for people with aphasia), and an instruction video for therapists about the sensor-feedback system. To share and make the knowledge accessible these developed multimedia materials could be integrated in an online platform. Within this online platform it would be interesting to create a database with example analogies (and other interventions) that could function as a source of inspiration for (future) therapists.

Furthermore, with regard to education, a distinction between bachelor students and more specialised tracks (graduate students or post HBO) may be made. For bachelor students, for whom implicit motor learning is integrated within the program, it may be easier to integrate implicit motor learning directly within their daily routines. Sufficient knowledge and experience with implicit and explicit motor learning creates the opportunity to make well informed decisions regarding the motor learning approach. However, for therapists with years of experience, in the beginning, it may be difficult to integrate implicit motor learning within daily life practice. Due to routines and habits, it is easy to fall back on, or to correct motor skills with detailed explicit instructions. In daily practice, many therapists may have already used some aspects of implicit motor learning e.g. through demonstration (action observation) or through using an analogy. However, often these strategies are combined with explicit instructions. It is easy to us an implicit instruction once, but the challenge lies in applying implicit motor learning in a pure and structured manner over a longer time period. Especially in the beginning, it requires some energy and a certain degree of creativity to keep therapy implicit of nature. It is harder than it seems to not use any explicit instructions at all.

\section{Implications for research}

Within this thesis, studies in a variety of settings, from the laboratory to clinical practice, were conducted. The trial within this thesis included a functional task, complex intervention and heterogeneous population. Future studies on implicit motor learning within neurological rehabilitation may consider different roads related to "identifying which patients benefit most from 
what motor learning approaches" and "examining whether the characteristics of implicit motor learning also apply for the neurological population".

\section{Participant characteristics: Which patients benefit most from what motor learning approach?}

To better understand the participant characteristics that play a role in (implicit and explicit) motor learning, future research should focus on a) identifying participant characteristics associated with motor learning approaches and b) to validate the identified factors (e.g. balance, attentional abilities and sensory functioning) across different populations and a variety of (functional) tasks within neurological rehabilitation. From a theoretical perspective and from recent literature, potential patient characteristics that play a role in implicit and explicit motor learning can be identified. From here, specific hypotheses for each participant characteristic in relation to implicit and explicit motor learning may be formulated and tested in a series of (more controlled) laboratory experiments. Knowing which patients benefit most from what approach, in combination with frameworks such as presented by Kleynen et al 41 can then help practitioners to make informed decisions regarding their motor learning approach.

Characteristics of the intervention: Do the typical characteristics of implicit motor learning also apply for the neurological population?

Implicit motor learning is characterised by robust performance during dual tasking, minimal accrual of explicit knowledge and stable performance over time. Due to the large variability in cognitive abilities we were not able to legitimately interpret our dual task results. When assessing working memory demands using dual tasks within the neurological population, future studies may consider the following options:

- Include a more homogenous sample in terms of cognitive abilities so that an appropriate dualtask difficulty can be selected. There are studies that suggest certain dual-tasks difficulties for different levels of cognitive abilities.194

- Include different dual tasks, such as spontaneous speech generation tasks e.g. "name as many animals as possible" or arithmetic tasks e.g. "count backwards in threes". As there is no ceiling effect in such tasks, they have the advantage that a more heterogenous population in terms of cognitive abilities can be included. However, people with aphasia cannot be included when using a dual task that requires speech. Also, attention should be payed to the type of dual task. Some tasks are more dependent on working memory and attention e.g. arrhythmic tasks, whereas others tasks may challenge different cognitive domains.

Another more direct way of assessing the cognitive processes that underlie motor learning is via using electroencephalography (EEG) or functional Near-Infrared Spectroscopy (fNIRS). Both measures can be used to measure cortical activity in real time. Various studies on implicit motor learning have used EEG to measure neural co-activation (coherence) e.g. Zhu et al195 or van Duijn et al15,196. Coherence measures simultaneous neural co-activation between two brain regions e.g. between the motor planning area and explicit, verbal analytical regions. There are EEG results during analogy learning that suggest reduced verbal processing demands.15 A strength of using EEG is that it provides a more reliable and objective measure. However, a disadvantage of EEG is that it is more complex and more difficult to use within clinical environments and functional tasks such as 
walking. It requires experts to both use the EEG and interpret the data. Furthermore, participants would have to carry and wear equipment which could potentially influence walking, especially for those with more severe impairments.

Another characteristic of implicit motor learning is that learning is assumed to take place without the accrual of explicit knowledge. Within our trial however, the therapists reported that some participants had already acquired explicit knowledge prior to the study. It would be interesting to explore if and how the presence of explicit knowledge affects, in particular, dual task performance. In healthy adults, the presence of explicit knowledge has been found to be detrimental for performance during multi-tasking or anxious situations.10-12 Is this also the case for people after stroke? And, what if people with explicit knowledge get exposed to an implicit motor learning intervention? Would it be possible to, in some way, reset (or replace) the 'default' (explicit, declarative) knowledge base with an analogy (at times that people would normally 'reinvest' in their movements)? 197 This is an interesting question as it is very likely that most people after stroke, especially in the chronic phase of recovery, already possess explicit, declarative knowledge.

\section{To conclude}

This thesis generated food for thought and inspiration for this challenging and relevant topic within neurological rehabilitation. Future steps may consider more fundamental and empirical studies to identify and validate participant characteristics and potential mechanisms of implicit motor learning, especially with regard to functional tasks and the neurological population. The interventions of these studies may be used as examples and as a source of inspiration for health care practitioners. Finally, I would like to conclude with reference to the title of this thesis "Different explanation, less frustration". Although 'frustration', was used by means of a metaphor, we can report that the participants within this thesis, including those with more severe cognitive deficits, were able to use the implicit instructions and found them relatively easy in use. Implicit motor learning walking interventions can be used in a feasible and effective manner to improve walking performance in people after stroke. 
128 | General Discussion 


\section{English Summary}


Physiotherapists teach patients motor skills on a daily basis. Motor learning in rehabilitation is a dynamic process and can be influenced by many factors such as pain, cognition or physical abilities, which can therefore result in challenging and complex situations. For people with acquired brain injury e.g. stroke patients, not only motor, but also cognitive functions may be impaired. As a consequence, understanding and processing explicit instructions about 'how' to perform a motor skill can be difficult. This manner of teaching (i.e. providing the learner exact step-by-step instructions how to execute a movement) is referred to as explicit motor learning. There are also other forms of learning, such as implicit motor learning, that are assumed to be less cognitively demanding. This thesis explored the application of implicit motor learning within neurological rehabilitation.

More specifically, the overall aim of this $\mathrm{PhD}$ thesis was to develop and explore the application of different implicit motor learning approaches and to assess the effectiveness and feasibility of one promising implicit motor learning approach at home for people after stroke. Additionally, the design of a user interface for a simple innovative technology that can be used to support independent practice of walking at home, independent of the approach, was developed.

To answer the overall aim, this thesis was divided into three phases. In phase 1 (chapters 2 and 3) we focused on exploring the application of different implicit motor learning approaches, which delivered input for the second phase. In phase 2 (chapters 4 to 6) a randomised controlled trial took place in which an implicit and explicit motor learning walking intervention for people after stroke were compared. Within these chapters, the protocol, results regarding the effectiveness and a process evaluation of the trial was presented. Finally, in phase 3 (chapter 7), the design process of a user interface for an existing sensor-feedback system that supports people after stroke during walking was described.

Chapter 1 introduced the topic and rationale of this thesis. First, implicit and explicit motor learning were defined and presented as two extremes on an implicit-explicit continuum. Then the characteristics of implicit motor learning were described, followed by an overview of different implicit motor learning strategies. Finally, the specific benefits of implicit motor learning for neurological rehabilitation were outlined. Additionally, a simple technology that supports patients during independent practice of walking at home was described.

Chapter 2 described whether analogy learning can be used to facilitate walking performance in people with Parkinson's disease. The analogy of 'following the footprints in the sand' was used to facilitate stride length and was practiced in eight walking trails. Walking performance (stride length and walking speed) was measured before and 
immediately after the intervention, and again 1-month later, using a CODA motion 3D analyses system. Patient experiences with the intervention were registered. The study revealed both statistically and clinically relevant improvements in walking performance, especially at 1 -month post intervention. Furthermore, a positive trend towards reducing dual task costs was demonstrated. Participants found the analogy relatively easy to use and it became easier to use over time. Analogy learning seemed feasible and potentially implicit (i.e. reduced working memory demands) to facilitate walking performance in people with Parkinson's.

Chapter 3 explored whether different implicit learning strategies can lead to immediate changes in walking performance. Associated patient experiences with each strategy were collected. People after stroke were randomly allocated to one of three implicit strategies: analogy instructions, environmental constraints, and action observation. The instructions within each condition were hypothesized to facilitate one specific gait parameter, either walking velocity, step length, step width, or step height. Gait parameters were recorded before and during the intervention using Vicon 3D motion analysis system. Performance at baseline was compared to performance during the conditions (within group differences). On a group level three of four analogies led to small but significant changes in velocity, step height and step width. Environmental constraints led to significant changes in step width. No significant changes were found within the action observation strategy. At an individual level, results showed a wide variation in magnitude of changes. Participants found it easy to walk with the different strategies. Analogy instructions and environmental constraints can be used to induce specific, immediate changes in walking performance. The researched strategies seem feasible but need to be tailored to the individual gait problem and preferences of the patients.

In chapters 4, 5 and 6, a trial was designed, conducted and evaluated. The trial examined whether an implicit motor learning walking intervention (analogies) is more effective compared to an explicit motor learning walking intervention (verbal detailed instructions) delivered at home with regard to walking speed in people after stroke.

Chapter 4 described the design for the trial. Within the trial only people after stroke who were in the chronic phase of recovery were included, to minimise the possibility for spontaneous recovery. Analogy learning was the leading strategy for the implicit intervention, whereas detailed explicit instructions were the leading strategy for the explicit intervention. The interventions took place over a 3-week period, consisted of 9 training sessions (3 per week), and each session lasted for 30 minutes. Walking performance was measured using the 10-meter walking test, and was assessed pre- and post- intervention, and again 1 month later. Intervention and measurement sessions took place at the home environment of the patients. The functional task, home environment and tailored motor learning interventions were specifically chosen to increase generalisability. 
To standardise the training content, therapists were trained and familiarised with the implicit and explicit conditions. A process evaluation was embedded to determine whether the interventions were delivered as intended and to explore the therapist and patient experiences with regard to feasibility.

In chapter 5, the results of the trial were presented. In total 79 people after stroke in the chronic phase of recovery took part in the study and received either an implicit $(n=38)$ or explicit $(n=41)$ motor learning walking intervention under guidance of a physiotherapist in their home environment. The implicit intervention had similar effects on walking speed compared to those who received an explicit intervention. No statistically nor clinically relevant differences were observed between groups. For people with relatively good cognitive abilities (MoCA >21), and people with more severe cognitive impairments $(\mathrm{MoCA} \leq 21)$ similar trends in performance were seen. Implicit motor learning was not superior to the explicit motor learning at improving walking speed in people after stroke in the chronic phase of recovery. The heterogeneous sample and personalised interventions that took place within clinical settings contributed to a high external validity. For tailored motor learning approaches more insight is needed on the patient characteristics and preferences that influence the process of motor learning.

Chapter 6 described the process evaluation of the trial with regard to the fidelity and feasibility of the interventions. Data for the process evaluation was collected alongside the trial by means of therapist logs, audio recordings, therapist and patient questionnaires. Overall therapists adhered to the intervention guidelines. However, data also showed that it is difficult to keep interventions $100 \%$ implicit or explicit. Incidentally implicit instructions were provided within the explicit intervention and vice versa $(14 \%$ of the implicit sessions; $1 \%$ of the explicit sessions). In total, two protocol deviations were observed within the implicit intervention. Therapists and participants were generally positive about the feasibility of both interventions but frequent comments were made by the therapists regarding "therapy time restrictions" and "tendency of the participants to develop explicit rules". The interventions were successful in terms of fidelity and overall therapists and patients were positive about the feasibility of the delivered implicit and explicit motor learning interventions within the trial.

Chapter 7 described how a usable and enjoyable user interface of a sensor-feedback system can be re-designed for people after stroke. The design process was structured around four consecutive design phases: the discovery, definition, development and delivery phase. Fifteen participants evaluated the prototypes in multiple test rounds, each existing of 2-7 individual test sessions. Deliverables and key observations derived from the design process were described. Apart from functional requirements and an understandable user interface, i.e., good usability, our case study demonstrated that the inclusion of a (fictional) character like "Stappy" may lead to a more meaningful and 
enjoyable user experience. The methodology, deliverables and key observations can be used to (re)design meaningful user interfaces for people after stroke. Through the creation of characters like Stappy, the study provides a technique that may promote "empathy". The description of the design process may provide guidance for health care professionals, researchers or designers in future user interface design projects in which existing products are redesigned for people after stroke.

Chapter 8 described the main findings of this $\mathrm{PhD}$ research within the three phases of this project. Subsequently, the methodological considerations related to three themes are discussed: 'order of chosen research designs', 'from isolated tasks in laboratory setting to clinical practice', and 'a structured process to design the user interface'. Furthermore, the lessons learned from this $\mathrm{PhD}$-trajectory are described within six major topics: 'the difficulty to determine effectiveness of implicit motor learning in clinical practice', 'personalised and meaningful analogies', 'tailoring motor learning: the importance of identifying participant characteristics', 'the need to understand implicit motor learning within the neurological rehabilitation', 'empathy through the creation of the character Stappy' and 'the value of patient participation'. Finally, this chapter described how knowledge transfer around implicit motor learning towards clinical practice and education can be achieved, and the current thesis contributed to this process. Potential directions for future research are discussed. It is argued that two important issues for future studies are "identifying which patients benefit most from what motor learning approaches" and "examining whether the characteristics of implicit motor learning also apply for the neurological population". 



\section{Nederlandse Samenvatting}


Fysiotherapeuten zijn dagelijks bezig om patiënten motorische vaardigheden aan te leren. Motorisch leren is een dynamisch proces dat door veel factoren zoals pijn, cognitie, en fysieke beperkingen kan worden beïnvloed. Dit kan leiden tot uitdagende en complexe situaties tijdens het (her)leren van motorische vaardigheden. Mensen met een nietaangeboren hersenletsel zoals een beroerte, hebben niet alleen te maken met fysieke beperkingen, maar ook met cognitieve problemen. Denk hierbij aan problemen met geheugen, aandacht of het verwerken van informatie. Hierdoor kan het begrijpen en verwerken van expliciete instructies over 'hoe' men een beweging moet uitvoeren, extra uitdagend en complex voor hen zijn. Het verbaal aanleren (i.e. de lerende stap-voor-stap instrueren hoe hij of zij een beweging moet uitvoeren) wordt ook wel expliciet leren genoemd. Er zijn ook echter ook andere leerstrategieën, zoals impliciet leren, die minder afhankelijk zijn van cognitieve functies van een patiënt.

Het hoofddoel van dit proefschrift was om verschillende impliciet motorische leerstrategieën voor het verbeteren van het lopen te ontwikkelen toe te passen en vervolgens de effectiviteit en hanteerbaarheid van één veelbelovende impliciete leerstrategie te vergelijken met expliciet motorisch leren. Daarnaast is er samen met de gebruikers een nieuwe userinterface van een simpele, innovatieve technologie ontwikkeld die mensen na een beroerte kan ondersteunen tijdens het zelfstandig oefenen van het lopen.

Om dit doel te behalen, is dit proefschrift opgedeeld in drie fasen. Fase 1 (hoofdstukken 1 tot en met 3) richt zich op de toepassing van verschillende impliciet motorische leerstrategieën. Hierbij is input gegenereerd voor de tweede fase. In fase 2 (hoofdstukken 4 tot 6) vond een gerandomiseerd gecontroleerd onderzoek plaats. Een impliciete en expliciete leerstrategie ter verbetering van het lopen bij mensen na een beroerte werden met elkaar vergeleken. In deze hoofdstukken worden het protocol, de effect- en procesevaluatie gepresenteerd. Tot slot is in fase 3 (hoofdstuk 7) het designproces evenals de eindversie van een userinterface voor een bestaand sensoren-feedback systeem beschreven, die mensen na een beroerte kan ondersteunen tijdens het zelfstandig oefenen van het lopen.

Hoofdstuk 1 introduceert het onderwerp en de doelstellingen van dit promotieonderzoek. Eerst worden impliciet en expliciet leren gedefinieerd en gepresenteerd als twee extremen op een impliciet-expliciet continuüm. Vervolgens worden de karakteristieken van impliciet motorisch leren beschreven, en wordt er een overzicht gegeven van verschillende impliciete motorische leerstrategieën. Tot slot worden de specifieke voordelen van impliciet motorisch leren voor de neurologische revalidatie toegelicht. Daarna worden de mogelijke voordelen van simpele technologieën voor het ondersteunen van het looppatroon in de (neuro)revalidatie beschreven. De inleiding eindigt met de overkoepelende doelstelling en de structuur van het proefschrift. 
In Hoofdstuk 2 staat de volgende onderzoeksvraag centraal: "Kan analogie leren worden gebruikt om het looppatroon bij mensen met de ziekte van Parkinson te faciliteren?". Analogie leren is een leerstrategie waarin alle biomechanische regels van een motorische vaardigheid zoals het lopen worden samengevat in één metafoor of analogie. Mensen met de ziekte van Parkinson konden deelnemen aan de studie indien zij een schuifelend looppatroon hadden, en zelfstandig, zonder loophulpmiddelen, konden lopen voor ten minste 15 minuten (met rust pauzes). Deelnemers zijn geworven via het netwerk van Parkinson UK. Alle deelnemers ontvingen de volgende interventie. De analogie "volg de voetstappen in het zand" werd samen met de foto (Figuur 1) aan de deelnemers gepresenteerd om de staplengte te faciliteren.

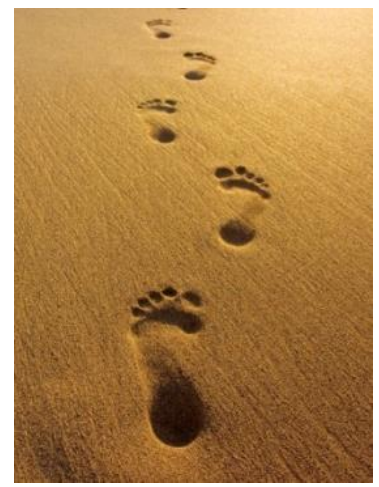

Figuur 1. Foto van voetstappen in het zand

Tijdens het lopen werd de instructie geoefend (acht keer 10 meter). Het looppatroon (loopsnelheid en lengte van de schrede) werd gemeten met behulp van een 3D bewegingsanalyse (CODA-motion) systeem. Het looppatroon werd gemeten tijdens het lopen (één taak) en tijdens het uitvoeren van een dubbeltaak (twee taken). Er waren twee dubbeltaken 1) lopen terwijl je een dienblad met lege kopjes draagt en 2) lopen terwijl men terugtelt in sprongen van drie. De uitvoering van het lopen zelf en de dubbeltaakprestaties werden gemeten voor de interventie, na de interventie en opnieuw één maand later. Daarnaast werd aan de deelnemende patiënten gevraagd hoe ze het gebruik van de analogie hadden ervaren.

In totaal hebben er twaalf mensen met de ziekte van Parkinson (zes mannen en zes vrouwen, met een gemiddelde leeftijd van 70 jaar, en een Hoehn en Yahr schaal variërend van I-III) deelgenomen aan de studie. De studie resulteerde in statistisch significante en klinisch relevante veranderingen in de loopsnelheid en lengte van de schrede. Deze veranderingen waren met name zichtbaar één maand na de interventie. Daarnaast was er een positieve trend richting betere dubbeltaakprestaties na de interventie zichtbaar. Dat veronderstelt dat het leerproces mogelijk impliciet van aard was. Deelnemers vonden de analogie over het algemeen makkelijk om te gebruiken en gaven aan dat dit makkelijker werd na verloop van tijd. Analogie leren lijkt een hanteerbare en mogelijk impliciete (i.e. 
minder belastend voor werkgeheugen) leerstrategie om het lopen bij mensen met Parkinsons te faciliteren.

In hoofdstuk 3 staat de volgende onderzoeksvraag centraal: "Kunnen verschillende impliciete leerstrategieën leiden tot specifieke, directe veranderingen in het looppatroon en hoe ervaren patiënten deze leerstrategieën?" Mensen konden deelnemen aan de studie indien zij een beroerte hebben gehad (minimaal drie maanden geleden), zelfstandig minimaal tien meter konden lopen met of zonder loophulpmiddel, en een hemiparese hadden (score < 100 op de Motricity Index (onderste extremiteiten); score < 34 Brunstrom Fugl-Meyer assessment (onderste extremiteiten)). Deelnemers zijn geworven via twee revalidatiecentra, één polikliniek (ziekenhuis) en via zeven fysiotherapiepraktijken. Deelnemers zijn gerandomiseerd in één van de drie impliciete leerstrategieën: analogie leren (er werd gelopen met beeldspraken), omgevingsaanpassingen (de omgeving werd aangepast door beelden te projecteren op de grond, bv. een zebrapad) of observationeel leren (er werden gekeken naar loopvideo's). Binnen iedere leerstrategie zijn verschillende instructies gebruikt behalve bij observationeel leren. Bij observationeel leren was er enkel één instructie, namelijk: "Bekijk de video, en probeer dit zo goed mogelijk na te doen". Iedere instructie had als doel om één specifieke loopparameter te veranderen, bijvoorbeeld de staphoogte: "Ziet u de diepe laag sneeuw? Doe als of u door deze diepe sneeuw loopt" (Figuur 2).

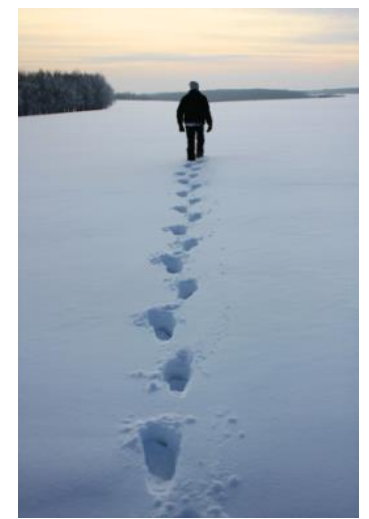

Figuur 2. Foto voetstappen in diepe sneeuw

De spatio-temporele loopparameters (loopsnelheid, staplengte, staphoogte en stapbreedte) werden vóór en tijdens de instructies gemeten met een 3D bewegingsanalyse systeem (VICON). Daarnaast zijn de ervaringen van de patiënten bij iedere leerstrategie genoteerd. Hierbij werd bijvoorbeeld gevraagd hoe moeilijk zij de instructies vonden. In totaal hebben 56 mensen ( 32 mannen en 24 vrouwen, met een gemiddelde leeftijd van 64 jaar, 28 links- en 28 rechtszijdig aangedaan, 16 mensen zonder en 40 met loophulpmiddel) deelgenomen aan de studie. Op groepsniveau resulteerde de analogie interventie $(n=19)$ in kleine significante veranderingen in loopsnelheid, staphoogte en stapbreedte. De 
interventie met omgevingsaanpassingen $(n=17)$ leidde tot significante veranderingen in stapbreedte. Er waren geen significante veranderingen in de spatio-temporele loopparameters binnen de interventie met observaties $(n=20)$. Deelnemers vonden het makkelijk om met de verschillende instructies te lopen, onafhankelijk van de toegewezen leerstrategie. Analogie leren en omgevingsaanpassingen kunnen leidden tot specifieke, directe veranderingen in het lopen. Er was echter sprake van grote variatie in veranderingen van het looppatroon. Daarnaast waren er soms ook negatieve associaties met de instructies, zoals "in het echte leven kan ik ook niet door sneeuw lopen met mijn rollator". De conclusie is dat de leerstrategieën hanteerbaar lijken maar afgestemd moeten worden op het individuele loopprobleem en de voorkeuren van de patiënten.

In Hoofdstuk 4 is het protocol voor een gerandomiseerd onderzoek met controlegroep (trial) beschreven. Dit hoofdstuk beschrijft de opzet en de gemaakte keuzes in het design van de trial. Om deel te nemen werden de volgende criteria gehanteerd. Om de kans op spontaan herstel te minimaliseren moesten deelnemers in de chronische fase na een beroerte zitten (minimaal zes maanden na de beroerte) en een hulpvraag met betrekking tot het verbeteren van hun looppatroon hebben. Mensen zijn geworven via lokale fysiotherapiepraktijken en revalidatiecentra en via een nieuwsitem in een lokale krant. Deelnemers werden door middel van randomisatie toegewezen aan een impliciete of expliciete interventie. Analogie leren was de gekozen leerstrategie om de impliciete interventie vorm te geven. Gedetailleerde, verbale, instructies was de gekozen leerstrategie om de expliciete interventie vorm te geven. Voor de therapeuten was er een interventie richtlijn waarin de interventies gedetailleerd zijn beschreven en alle therapeuten ontvingen trainingssessies voor het geven van de interventies. Alle instructies werden afgestemd op het individuele loopprobleem en de individuele achtergrond (bv. hobby's en ervaringen) van de patiënt. De interventies vonden plaats over een periode van 3 weken, bestonden uit 9 trainingssessies (3 per week) en iedere sessie duurde 30 minuten. De loopsnelheid als hoofduitkomstmaat werd gemeten met de 10 meter wandel test, voor de interventie, na de interventie en één maand na de interventie. Daarnaast zijn er secundaire uitkomstmaten meegenomen zoals de uitvoering van dubbeltaakprestaties. De interventies en metingen vonden plaats in de thuisomgeving van de deelnemers.

$\mathrm{Er}$ is een procesevaluatie ingebed binnen dit onderzoek om te bepalen of de interventies zijn uitgevoerd zoals bedoeld en om de therapeut- en patiëntervaringen met betrekking tot de hanteerbaarheid van de interventies te onderzoeken. Om de generaliseerbaarheid naar de dagelijkse praktijk te vergroten is specifiek gekozen voor het oefenen van een functionele taak (lopen), trainingen in de thuisomgeving en gepersonaliseerde interventies. In deze studie is extra aandacht gegeven aan het bewaken van het contrast tussen de interventies. Er is duidelijk beschreven hoe de twee interventies zijn opgebouwd en hoe zij van elkaar verschillen. 
In hoofdstuk 5 staat de volgende onderzoeksvraag centraal: "Is een 3-weken durende impliciet motorische loopinterventie aan huis effectiever dan een 3-weken durende expliciet motorische loopinterventie aan huis ter verbetering van de loopsnelheid bij mensen na een beroerte?. Mensen met een beroerte konden deelnemen aan de studie indien zij zich in de chronische fase na een beroerte bevonden $(<6$ maanden na de beroerte), hun looppatroon wilden verbeteren, zij een lage loopsnelheid $(<1.0 \mathrm{~m} / \mathrm{s})$ hadden, Nederlands spraken, en een 3-delige instructie konden voltooien. Mensen zijn geworven via lokale fysiotherapiepraktijken en revalidatiecentra, en via een nieuwsitem in een lokale krant. Voor de therapeuten was er een interventie richtlijn waarin de interventies gedetailleerd staan beschreven en alle therapeuten ontvingen trainingssessies voor het geven van de interventies. Deelnemers werden gerandomiseerd in een impliciete of expliciete interventie.

In totaal zijn er 79 mensen na een beroerte in chronische fase geïncludeerd. Van deze groep ontvingen 38 mensen een impliciete en 41 mensen een expliciete loopinterventie. Deelnemers in de impliciete interventie (verbetering van $0.08 \mathrm{~m} / \mathrm{s}$ ) lieten vergelijkbare effecten zien op loopsnelheid ten opzichte van deelnemers die een expliciete interventie (verbetering van $0.06 \mathrm{~m} / \mathrm{s}$ ) ontvingen. Op alle uitkomstmaten zijn geen statistische of klinische relevante verschillen tussen de groepen gevonden. Vergelijkbare trends in loopprestaties waren zichtbaar voor mensen zonder grote cognitieve beperkingen (MoCA $>21)$ en voor mensen met meer cognitieve beperkingen $(\mathrm{MoCA} \leq 21)$.

De heterogene groep en de gepersonaliseerde interventies die plaatsvonden in een klinische setting droegen bij aan een hoge externe validiteit. Echter, deze factoren hebben mogelijk ook bijgedragen aan een verminderd contrast tussen de interventies. Voor gepersonaliseerde interventies zijn meer inzichten nodig in de patiëntkarakteristieken en voorkeuren van de patiënt die het proces en daarmee het effect van motorisch leren beïnvloeden.

Hoofdstuk 6 presenteert de resultaten van de procesevaluatie die parallel aan de effectstudie is uitgevoerd. Hierin staan de volgende onderzoeksvragen centraal "In welke mate zijn de interventies uitgevoerd zoals bedoeld (protocol-navolging, fidelity)? Hoe hebben therapeuten de toepassing van interventies ervaren op gebied van hanteerbaarheid? Hoe hebben patiënten de interventies die zij ontvingen ervaren?" Zowel de therapeuten als deelnemers hebben deelgenomen aan de procesevaluatie. Ervaren therapeuten ( $>10$ jaren) binnen de neurologische revalidatie en/of experts op het gebied van motorisch leren hebben de interventies gegeven. Alle therapeuten hebben zowel de impliciete als expliciete loopinterventies gegeven. Data met betrekking tot protocol-navolging werd verzameld middels de therapeutenlogs en audio-opnames. Ervaringen met betrekking tot de hanteerbaarheid van de interventies werden verzameld middels vragenlijsten voor therapeut en patiënt. 
In totaal waren er 79 deelnemers waarvan er zeven deelnemers zijn uitgevallen (drie deelnemers hadden geen hulpvraag met betrekking tot het lopen, drie deelnemers hadden andere gezondheidsredenen (niet gerelateerd aan lopen) waardoor zij niet door wilden met de interventie). Hierdoor hebben er in totaal 72 deelnemers deelgenomen aan de procesevaluatie. Alle deelnemers ontvingen zeven of meer therapiesessies zoals voorgeschreven in het protocol. In totaal zijn er bij twee deelnemers binnen de impliciete groep protocoldeviaties geobserveerd omdat binnen deze deelnemers in meer dan twee sessies een expliciete instructie was gebruikt. Er zijn geen protocol deviaties geobserveerd binnen de expliciete groep. Incidenteel (in minder dan twee sessies per deelnemer) werden er expliciete instructies gebruikt in impliciete interventie en vice versa.

Therapeuten en patiënten waren over het algemeen positief over de hanteerbaarheid van beide interventies. Door therapeuten werden regelmatig opmerkingen beschreven met betrekking tot "restricties in therapietijd", "de neiging van deelnemers om expliciete regels te gebruiken" en "veranderingen in het lopen". Therapeuten benoemden bijvoorbeeld dat de eerste sessies van 30 minuten soms krap waren, later wanneer een goede analogie gevonden was, was deze tijd voldoende. Daarnaast werd ook vaker benoemd, dat deelnemers soms hun eigen looppatroon analyseerde waardoor zij zelf expliciete regels ontwikkelde of expliciete regels werden opgedaan doordat partners de analogieën vertaalden naar expliciete regels. Geconcludeerd kan worden dat impliciet en expliciet motorische loopinterventies zoals aangeboden in de trial hanteerbaar zijn. De studie liet zien dat het moeilijk is om de interventies $100 \%$ impliciet of expliciet te houden. Voor de praktijk is het belangrijk dat alle (informele) zorgverleners op een lijn zitten qua behandeling. Daarnaast zijn er veel factoren zoals de neiging van een patiënt om zelf expliciete regels te ontwikkelen, die de mate van een impliciete of expliciete interventie bepalen.

In hoofdstuk 7 staat de volgende onderzoeksvraag centraal "Hoe kan een bruikbare en plezierige user-interface worden ontworpen voor een sensoren-feedback systeem gericht op mensen na een beroerte die leidt tot een betekenisvolle gebruikerservaring? Binnen deze studie werd de user-interface van een bestaand sensoren-feedbacksysteem (her)ontworpen op basis van de voorkeuren en behoefte van mensen na een beroerte. Het sensoren-feedbacksysteem kan mensen ondersteunen tijdens het zelfstandig oefenen in de thuissituatie. Eerst zijn gebruikerseisen geïdentificeerd, zoals leesbaarheid en contrast van informatie (oudere doelgroep), begrijpen, onthouden en verwerken van informatie (beperkingen in cognitie) en mogelijke fysieke beperkingen (beperkingen van motorische vaardigheden). Hierna werd gestart met de test-sessies. Mensen konden deelnemen indien zij cognitieve beperkingen ervaarden (omdat dit als gebruikerseis geïdentificeerd was), open stonden voor het gebruik van technologie, en hun looppatroon wilden verbeteren. Deelnemers zijn geworven via een revalidatiecentrum en via cliëntvertegenwoordigers van het project. Het designproces was gestructureerd in vier opeenvolgende fasen: de 
ontdekkings-, definitie-, ontwikkel- en de opleveringsfase. Vijftien deelnemers hebben de prototypes in verschillende testrondes, bestaande uit twee tot zeven individuele testsessies, geëvalueerd. In deze testrondes zijn de volgende deliverables geëvalueerd: een lijst met gebruikerseisen, een persona, een user-flow, een low-, medium- en high-fidelity prototype en het karakter "Stappy" (i.e. een soort mascotte, die de gebruiker door alle stappen in de app meeneemt). Uit de testsessies blijkt dat, naast een functioneel werkend systeem en een begrijpbare userinterface i.e., een goed bruikbaar systeem, de inclusie van een karakter zoals "Stappy", mogelijk kan leiden tot een meer betekenisvolle en plezierige gebruikerservaring. Methodologie, producten, en hoofdobservaties kunnen gebruikt worden bij het (her)ontwerpen van toekomstige producten en userinterfaces voor mensen na een beroerte. Via de creatie en integratie van karakters zoals "Stappy", biedt deze studie mogelijk een techniek om "empathie" te promoten in user-interface designs.

In Hoofstuk 8 begint met de beschrijving van de belangrijke bevindingen en discussies binnen dit proefschrift. Daarna worden methodologische keuzes met betrekking tot de volgende drie onderwerpen bediscussieerd: "Volgorde van de gekozen onderzoeksdesigns", "van geïsoleerde taken in een labsetting naar de klinische praktijk", en "een gestructureerd designproces van een userinterface". Hierna zijn de geleerde lessen beschreven. Deze zijn geclusterd in zes hoofdthema's, te weten: "de moeilijkheid om de effectiviteit van impliciet motorisch leren binnen de klinische praktijk te bepalen", "gepersonaliseerde en betekenisvolle analogieën", "motorisch leren personaliseren: het belang van het identificeren van persoonskarakteristieken", "het belang om impliciet motorisch leren binnen de neurologische revalidatie te begrijpen" "het creëren van empathie via de creatie van het karakter Stappy". Tot slot beschrijft dit hoofdstuk hoe kennis rondom impliciet motorisch leren kan worden vertaald naar de klinische praktijk en het onderwijs en op welke manier de kennis van dit promotieonderzoek hieraan een bijdrage kan leveren. Daarbij worden aanbevelingen gedaan voor mogelijk toekomstige onderzoeken en wordt bediscussieerd waarom toekomstige studies zich meer zouden moeten focussen op "het identificeren van patiënt karakteristieken passen bij impliciet en explicit leren" en "de karakteristieken van impliciet motorisch leren binnen de neurologische populatie nader onderzoeken". 


\section{Valorisation}


Valorisation has been defined by the Dutch government as "the process of creating value from knowledge by making knowledge suitable and/or available for economic and/or societal use and translating that knowledge into products, services, processes and entrepreneurial activity".198 In other words obtained knowledge from research projects, such as generated within this $\mathrm{PhD}$ thesis, becomes especially valuable when it is used in society. For example, results of this $\mathrm{PhD}$ thesis can be relevant to patients, (informal) caregivers, (future) health care professionals but also to technology manufacturers, designers, or policy makers. They can use the knowledge in daily life practice (e.g. patients and health care professionals) or the results may deliver input for future projects (e.g. technology manufacturers, designers or researchers) and the development of guidelines (e.g. policy makers). However, the generation of scientific knowledge does not mean that it automatically reaches society.

The following chapter describes how the findings of this thesis are relevant for society. Firstly, the overall relevance of the clinical problem is described. Then the chapter outlines how the findings of this thesis are relevant for different stakeholders and how the findings have already been disseminated. Finally, the future dissemination activities and innovative nature of the $\mathrm{PhD}$ project are described

\section{Relevance of the clinical problem}

Every year there are many people who suffer from the consequences of an acquired brain injury or from chronic neurological disorders. For example, in the Netherlands alone, there are about 500.000 people who suffer from a stroke and about 50.000 people with Parkinson's disease in a total population of about 17 million inhabitants.199,200 These conditions can lead to a wide variety of impairments, such as problems with physical, or cognitive functions, which in turn can affect daily life activities. As a consequence, many people need to (re)learn daily life motor skills such as walking, standing up from a chair or taking the stairs. The ability to perform these activities in a safe and independent manner, are important for autonomy and are seen as important factors for discharge from hospitals or rehabilitation facilities.

Health care professionals such as physiotherapists and occupational therapists are constantly searching for the best treatment approaches for their patients. However, the process of motor learning in neurological rehabilitation is dynamic and can be influenced by many factors such as pain, cognition or physical abilities, which can therefore result in challenging and complex situations. To deliver effective and efficient therapy it is important to know which motor learning approaches are feasible and effective within clinical practice. Findings of the current thesis add to this field of research and clinical practice as the developed interventions within this thesis demonstrate how implicit and motor learning can be structured within clinical practice and the results provide insight in the effects and feasibility of the researched motor learning strategies. 


\section{Target population and other stakeholders}

The findings of this thesis are valuable to a variety of stakeholders as described below.

\section{Patients and (informal) caregivers}

The knowledge obtained from this thesis can be of benefit to patients who are in the process of (re)learning motor skills. This knowledge may also be relevant to (informal) caregivers e.g. partners who provide support during this process. Results indicate that individually tailored implicit and explicit motor learning approaches can both be used to facilitate motor skills such as walking in in a feasible and potentially effective manner. Importantly, overall, independent of the cognitive abilities, patients were able to use the instructions, and were satisfied about the received interventions. To avoid confusion and to ensure that everyone is supporting the patient in the same manner, it is alaso necessary to inform the caregiver (and other professionals who treat the patient) about the chosen treatment approach. For example, if an implicit strategy turned out to work well for the patient therapy should not be disturbed with explicit instructions. Simultaneously, if an explicit approach or mix of learning strategies are being used this should be clear for everyone who supports the patient during the (re)learning process of motor skills.

Furthermore, patients who took part in the study may have benefitted from the sensorfeedback system "Stappy". The system provides the possibility to practice walking independently at times that are convenient for the patient. Additionally, the system provides the opportunity to receive feedback about their performance also outside the guided therapy sessions. At this moment, the sensor-feedback system with Stappy userinterface is not commercially available. However, patients may consider technologies with similar functions such as activity trackers that provide feedback (perhaps less specific compared to "Stappy") about walking performance e.g. about the walking distance or walking speed. Feedback about own movement performance can motivate people to practice more.201

\section{Health care professionals and clinical practice}

The current $\mathrm{PhD}$ thesis focused on improving walking performance and findings are therefore relevant to health care professionals that support patients during this specific activity. However, the knowledge that we have generated may also be useful during the execution of other daily life motor skills such as taking the stairs, dressing or for example during any sports related activities. Findings, may therefore be useful to a wide variety of disciplines such as physiotherapists, occupational therapists, nurses, and sports coaches. The motor learning framework as developed by Kleynen et al 4 provided a potential structure for motor learning strategies that can be viewed on an implicit-explicit continuum. The current project builds on this framework through providing more knowledge on how implicit (mainly analogy learning) and explicit motor learning 
approaches can be applied within clinical practice. Results have led to knowledge regarding the feasibility and potential effects of implicit and explicit motor learning within clinical practice. For health care professionals, it is especially important to know 'how' and 'when' these motor learning approaches can be applied. The interventions presented in the pilot studies (chapters 2 and 3) and in the trial (chapters 4 to 6) provide concrete examples of how implicit and explicit motor learning can be applied to facilitate walking performance. The process evaluation of the trial presents example instructions (chapter 6 ) that have been used within the interventions and provides insight in the factors that play a role in the application of implicit or explicit motor learning in practice. This concrete knowledge and information can be used by health care professionals as a guidance and source of inspiration.

\section{Education (students)}

Evidence Based Practice (EBP) plays a central role in education and clinical practice. Students learn to make decisions based on the available evidence, their own experiences and the preferences and backgrounds of the client. However, students generally lack experience and expertise in clinical practice, which makes it hard to make decisions based on earlier experiences or to recognize certain patterns. Therefore, especially in the beginning, they mainly fall back on the available scientific evidence that is published in (peer-reviewed) journals or summarised in evidence-based guidelines. Current guidelines in neurological rehabilitation describe the importance of motor learning principles such as sufficient dose, intensity, task and context specific training e.g. see 128 . However, generally these guidelines do not describe "how" and "when" implicit and explicit motor learning can best be applied. Kleynen et al4 developed a motor learning framework that presents a potential structure and insights in different motor learning strategies and how these can be viewed on an implicit-explicit continuum. Through the scientific publications with descriptions and examples of the interventions, this study adds extra knowledge for students on especially how implicit and explicit motor learning can be applied in clinical practice.

\section{Education (Lecturers)}

Results of this thesis are relevant for lecturers as the generated knowledge and materials provide extra tools to structure lectures and practical lessons. Various materials have been developed such as video's with patient and therapist experiences, knowledge clips about motor learning and workshops (see dissemination). The use of such materials may perhaps contribute to more enjoyable and engaging teaching and learning environments. 


\section{Researchers}

The findings of this thesis contribute to the overall body of knowledge with regard to motor learning in neurological rehabilitation. Results are relevant to researchers as they bring new implications and directions for future research. From this $\mathrm{PhD}$ project several research questions for future research have emerged. For example, both interventions seemed equally effective, however it would be interesting to explore which patient(s) (characteristics and preferences) benefit most from which motor learning intervention (implicit or explicit). Furthermore, the project also provided insights in the complexity that goes accompanied with research in real life settings, complex tasks and a representative sample. Looking back on the lessons learned, researchers may consider studying these patient characteristics in relation to motor learning in more controlled environments. Ultimately it would be interesting to map all patient characteristics and identify their relationship to (e.g. implicit and explicit) motor learning so that in the end therapists can make better informed decisions regarding their chosen motor learning approaches.

If future research projects would consider randomised controlled trials to assess the effects of implicit motor learning for stroke survivors, more insight is needed in the patient characteristics and preferences of the patient. Furthermore, researchers should carefully consider the research setting i.e. laboratory or clinical practice and potential factors that can influence the contrast between the intervention. Researchers may also consider different research designs to gain insights in the application and effects of implicit motor learning such as cohort studies in which many potential influencing factors are measured over time or using multiple baseline study designs.

This $\mathrm{PhD}$ project was part of a larger project "the power of implicit motor learning 2.0" and currently the research on this topic will be continued in the form of two new followup projects. One project will focus on creating instruction and example material for (informal) caregivers, whereas the other project will focus on gaining more insight in the factors that play a role during the process of motor learning.

\section{Technology manufacturers and designers}

Today there are many supportive technologies that can be used by patients during their rehabilitation, for example activity trackers, smartphone apps or e-health platforms. However, even though (supportive) technologies generally function and operate perfectly on a 'technical' level, they are not always used as regularly as intended. There are many factors that determine the actual level of use of the (supportive) technologies174. For example, one of the reasons may be that technologies are not inviting to use for example because the user-interface is too complex, boring or because the users lack proficiency in the English language. The current $\mathrm{PhD}$ thesis (chapter 7) demonstrates how user-interfaces can be designed using a user-centered approach. Using this approach in design processes 
will hopefully lead to developments of user-interfaces that will lead to a more meaningful user experience. Furthermore, the systematic description of the user-centred approach and the associated deliverables may hopefully act as a guidance to future projects that (re)design or develop products for the stroke population.

\section{Policy makers}

The knowledge obtained within this $\mathrm{PhD}$ thesis can be relevant to policy makers who are active in the development of guidelines in neurological rehabilitation. The knowledge of this thesis adds to current guidelines as it provides more evidence and insight in 'how' and 'when' implicit and explicit motor learning can be applied. This current PhD project delivered knowledge in terms of 1) scientific evidence for implicit and explicit motor learning and 2) the practical application and feasibility of these motor learning strategies in neurological rehabilitation. The trial (chapter 3 to 6) showed 'how' tailored implicit and explicit motor learning can be applied in an effective and feasible manner to people after a stroke. However, in order to determine who benefits most from which intervention more insight is needed in the patient characteristics and working mechanisms of implicit motor learning. The process evaluation of the trial (chapter 6) also provides insight in a variety of factors that may play a role during implicit and explicit motor learning. Furthermore, this $\mathrm{PhD}$ project also showed that even people after stroke who are in the chronic phase of recovery (> six month after stroke) still have the ability to obtain meaningful improvements in short 'boost' sessions as presented within this trial. 


\section{Dissemination}

Tables 1 to 4 describe the dissemination activities performed so far for patients and society, health care professionals and clinical practice, education and the research community.

Table 1. Overview of dissemination activities for patients and society

\begin{tabular}{|c|c|}
\hline & Knowledge transfer to patients and society \\
\hline Factsheets & \multirow{3}{*}{$\begin{array}{l}\text { Dutch: Onderzoeksbeschrijving "De kracht van het onbewuste leren 2.0" } \\
\text { English: Research description "The Power of Implicit Motor Learning 2.0" } \\
\text { To read the factsheets scan the } Q R \text { code. }\end{array}$} \\
\hline 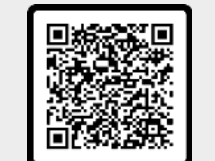 & \\
\hline SCAN ME & \\
\hline Symposia & \multirow{3}{*}{$\begin{array}{l}\text { The project end symposium. June 2019. To watch the aftermovie of the symposium } \\
\text { scan the QR code. }\end{array}$} \\
\hline 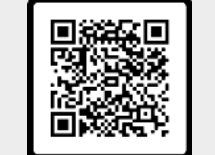 & \\
\hline SCAN ME & \\
\hline $\begin{array}{l}\text { Dissemination } \\
\text { through client } \\
\text { representatives }\end{array}$ & \multirow{4}{*}{$\begin{array}{l}\text { Else de Bont, Anja Minheere and Nathalie Sieben. Participerende patiënten aan het } \\
\text { woord. Workshop op eind-symposium van het project. June } 2019 \\
\text { Anja Minheere. Het is alleen zinvol als het goede participatie is. Publication in } \\
\text { burgkracht. May 2019. To read the article scan the QR code. } \\
\text { Else de Bont, Anja Minheere Nathalie Sieben. Cliëntenparticipatie, de waarde van } \\
\text { ervaringsdeskundigheid. Publication in Zorgbelang. December } 2015\end{array}$} \\
\hline 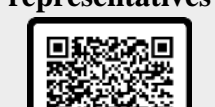 & \\
\hline 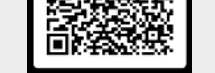 & \\
\hline SCAN ME & \\
\hline \multirow[t]{4}{*}{$\begin{array}{l}\text { Newspaper } \\
\text { reports }\end{array}$} & $\begin{array}{l}\text { De Limburger. "Zuyd Hogeschool wint landelijke prijs voor onderzoek naar } \\
\text { revalidatie na een beroerte". November } 2019\end{array}$ \\
\hline & $\begin{array}{l}\text { Sittard-Geleen nieuws. "Onderzoeken Zuyd Hogeschool vallen in de prijzen". } \\
\text { November } 2019\end{array}$ \\
\hline & 1limburg. "Opnieuw publieksprijs voor Zuyd: leren lopen met beeldspraak". \\
\hline & November 2019 \\
\hline $\begin{array}{c}\text { Radio } \\
\text { appearances }\end{array}$ & \multirow[t]{3}{*}{$\begin{array}{l}\text { RTV Maastricht. 'Station Maastricht'. } 12 \text { November 2019. To watch the episode scan } \\
\text { the QR code. }\end{array}$} \\
\hline 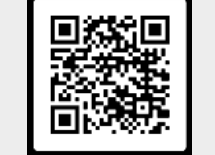 & \\
\hline SCAN ME & \\
\hline
\end{tabular}


Table 2. Overview of dissemination activities for health care professionals and clinical practice

\begin{tabular}{|c|c|}
\hline \multicolumn{2}{|c|}{ Knowledge transfer to health care professionals and clinical practice } \\
\hline $\begin{array}{l}\text { Presentations at } \\
\text { national } \\
\text { conferences and } \\
\text { symposia }\end{array}$ & $\begin{array}{l}\text { Jie LJ, Kleynen M, Meijer K, Beurskens AJ, Braun SM. Implicit and explicit motor } \\
\text { learning in gait rehabilitation of people after stroke: A randomized controlled single } \\
\text { blind trial. (Poster presentation at the Dag van de Fysiotherapeut, Den Bosch, the } \\
\text { Netherlands, November 2019). } \\
\text { Jie LJ, Kleynen M, Meijer K, Beurskens AJ, Braun SM. (2019) Implicit and explicit } \\
\text { motor learning in gait rehabilitation of people after stroke: a randomized controlled } \\
\text { single blind trial (Poster presentation at de dag voor de fysiotherapeut, Den Bosch } \\
\text { November 2019) } \\
\text { Jie LJ. Impliciet motorisch leren met behulp van analogieën. (Oral presentation at the } \\
\text { symposium 'Motorisch leren in de neurorevalidatie: van theorie naar therapie'. Wijk } \\
\text { aan zee, Februari 2019) } \\
\text { Jie LJ. Looptraining bij mensen na een beroerte: de toepassing van impliciet } \\
\text { motorisch leren en het gebruik van een sensoren feedback systeem. (Oral presentation } \\
\text { at the Jubileum conference of Key Point neurorevalidatie. Utrecht, October 2019) } \\
\text { Jie JL. Nooit meer expliciet? (Oral presentation at the Afscheidsreceptie Frank van } \\
\text { Hartingsveld. Amsterdam, November 2019) } \\
\text { Jie JL. Impliciet motorisch leren bij het verbeteren van de loopvaardigheid bij mensen } \\
\text { na een beroerte. Hoe doe je dat? (Oral presentation at the Symposium "Motorisch } \\
\text { leren in sport en revalidatie: wat kunnen we van elkaar leren?”. Maastricht, April } \\
\text { 2018) }\end{array}$ \\
\hline $\begin{array}{l}\text { Publications in } \\
\text { national } \\
\text { professional } \\
\text { journals }\end{array}$ & $\begin{array}{l}\text { Jie LJ, van den Heuvel R, Braun SM, Kleynen M. Looptraining met behulp van } \\
\text { technologie; ervaringen van CVA-patiënten en therapeuten met het sensoren-feedback } \\
\text { systeem 'Stappy'. Keypoint Tijdschrift voor behandelaars in de neurorevalidatie. } \\
\text { 2020;43E(3):16-19. }\end{array}$ \\
\hline $\begin{array}{l}\text { Implementation } \\
\text { in routine care } \\
\text { through } \\
\text { workshops for } \\
\text { health care } \\
\text { professionals }\end{array}$ & $\begin{array}{l}\text { Workshops on implicit motor learning and the application of the sensor-feedback } \\
\text { system Stappy within neurological rehabilitation. For physiotherapists working within } \\
\text { neurological rehabilitation. } \\
\text { At Zuyd University of Applied Sciences } \\
\text { - Workshop 1: } 03-09-2018 \\
\text { - Workshop 2: 20-09-2018 } \\
\text { At Adelante Rehabilitatie Centre. } \\
\text { - Workshop 1: 01-11-2018 } \\
\text { - Workshop 2: } 24-11-2018\end{array}$ \\
\hline $\begin{array}{l}\text { Intervention } \\
\text { guideline of the } \\
\text { trial }\end{array}$ & $\begin{array}{l}\text { Kleynen M, Jie LJ, Theunissen K, Halfens J, Kurvers J. Raamwerk voor de toepassing } \\
\text { van impliciet en expliciet motorisch leren. Januari } 2017\end{array}$ \\
\hline Video clips & $\begin{array}{l}\text { One instruction video of the sensor-feedback system has been developed for } \\
\text { healthcare professionals. }\end{array}$ \\
\hline
\end{tabular}


Table 3. Overview of dissemination activities within education

\begin{tabular}{l|l}
\hline \multicolumn{1}{|c}{ Knowledge transfer to education } \\
\hline Lectures & $\begin{array}{l}\text { Learning strategies for gait rehabilitation in course. Lecture at the Master track } \\
\text { Human Movement Sciences. Maastricht University. 2017, 2018, and } 2019 .\end{array}$ \\
& $\begin{array}{l}\text { Technologie in de zorg. Lecture at the bachelor track Physiotherapy. Zuyd } \\
\text { University of Applied Sciences. 2017 } \\
\text { Motorisch leren binnen de neurorevalidatie. Lecture at the minor "growing older". } \\
\text { Zuyd University of Applied Sciences. 2015 and 2016. }\end{array}$ \\
\hline Video clips & $\begin{array}{l}\text { In total 13 short video clips on the topic of (implicit) motor learning have been } \\
\text { recorded and made available to students and staff from Zuyd University of Applied } \\
\text { Sciences. }\end{array}$ \\
$\begin{array}{l}\text { One video clip with patient experiences on implicit motor learning has been } \\
\text { developed }\end{array}$ \\
$\begin{array}{l}\text { Inclusion of students } \\
\text { in graduation } \\
\text { projects }\end{array}$ & $\begin{array}{l}\text { One video clip with patient experiences of the sensor-feedback system Stappy has } \\
\text { been developed }\end{array}$ \\
\hline $\begin{array}{l}\text { A total of 48 students from different disciplines such as physiotherapy, } \\
\text { communication multimedia design and human movement sciences were involved } \\
\text { in 18 thesis projects. }\end{array}$
\end{tabular}

Table 4. Overview of dissemination activities within the research community

\begin{tabular}{|c|c|}
\hline \multicolumn{2}{|r|}{ Knowledge transfer to research community } \\
\hline $\begin{array}{l}\text { Publications in peer- } \\
\text { reviewed journals }\end{array}$ & $\begin{array}{l}\text { Four out of six articles included in this thesis have been published in international, } \\
\text { peer-reviewed journals. Two articles have been submitted for publication. }\end{array}$ \\
\hline $\begin{array}{l}\text { Presentations at } \\
\text { (inter)national } \\
\text { scientific } \\
\text { conferences }\end{array}$ & $\begin{array}{l}\text { The project results have been presented and discussed at (inter)national } \\
\text { conferences e.g. Dutch Congress of Rehabilitation Medicine (2016), Congress on } \\
\text { Neurorehabilitation and Neural Repair (2015 \& 2019), and the ISPGR World } \\
\text { Congress (2019). }\end{array}$ \\
\hline Follow-up grants & $\begin{array}{l}\text { Top-Up project: A project that aims to translate the knowledge into instruction } \\
\text { and example material for (informal)caregivers to provide better support to the } \\
\text { patient during the process of (implicit)motor learning process. } \\
\text { The ACTIE project: ACTIE stands for an approach for the complexity of in } \\
\text { neurological rehabilitation (in Dutch: Aanpak van Complexiteit neurorevalidaTIE). } \\
\text { The project aims to identify which factors play an important role during the } \\
\text { process of successful motor learning. }\end{array}$ \\
\hline Multimedia & $\begin{array}{l}\text { ResearchGate: } \\
\text { • Project “The-power-of-implicit-motor-learning-20”( } 58 \text { Followers) } \\
\text { - Personal account “Li-Juan_Jie" (133 Followers) } \\
\text { Twitter accounts: } \\
\text { • Project @ zuyd_mind (169 Followers) } \\
\text { - Personal account @ } 1 \text { lijuanjie (305 Followers) } \\
\text { Project website: www.m-i-n-d.org }\end{array}$ \\
\hline
\end{tabular}




\section{Future dissemination and implementation activities}

The following chapter describes the future dissemination and implementation activities per target population.

\section{Knowledge transfer to patients and society}

Dissemination of results towards society will continue through the start of a new project (Top-Up grant from Nationaal Regieorgaan Praktijkgericht Onderzoek SIA). This project aims to develop instruction and example material that is specifically focused on informal caregivers e.g. partners or family members. This material can be used to improve the process of (implicit) motor learning at home and in their social environments and may strengthen the overall rehabilitation trajectory. Furthermore, results of this $\mathrm{PhD}$ thesis will be disseminated through press releases at Zuyd University of Applied Sciences and Maastricht University. The $\mathrm{PhD}$ thesis will be freely accessible through the research repository of Maastricht University (https://cris. maastrichtuniversity.nl/portal/).

\section{Knowledge transfer to health care professionals and clinical practice}

Knowledge may be transferred through presentations at national conferences and symposia targeted at health care professionals. In 2020, an article about the results of this $\mathrm{PhD}$ project will be published in the Journal of Physiotherapy of the Royal Dutch Society for Physical Therapy. Through this publication, project results will be communicated to a wide range of physiotherapists in the Netherlands.

\section{Knowledge transfer to education}

Various materials have already been developed in the form of scientific publications and developed materials (e.g. video clips) and are currently integrated in a variety of courses at Zuyd University of Applied Sciences e.g. the Physiotherapy, and Occupational Therapy tracks. Results are also being taught within other tracks of other (inter)national universities such as the University of Exeter, Waikato University and Fontys University of Applied Sciences. Future dissemination to education will continue in this manner and the development of new material (e.g. through the Top-Up grant) may lead to a larger database of educational resources on the topic of motor learning.

\section{Knowledge transfer to the research community}

Knowledge transfer to the research community will continue through setting up new research projects. In 2020 one research project will start to explore and identify which factors play a role during the process of motor learning of movements in people after stroke. Furthermore, knowledge will be transferred to the research community through a research visit (through the YERUN mobility award) that will take place in April 2020. Through this visit, knowledge on motor learning and research methodologies will be exchanged between Brunel University London, Maastricht University and Zuyd 
University of Applied Sciences, which may lead to new collaborations. Finally, there are plans to start new research projects that focus on gaining a better understanding of the specific patient characteristics and their relationship to motor learning.

\section{Innovative aspects}

At the start of my $\mathrm{PhD}$ in 2015, evidence for implicit motor learning was almost exclusively based on studies in cognitive psychology and sports populations. However, the application and potential benefits of implicit learning seemed also promising for neurological rehabilitation. Therefore, findings are innovative of nature. The project contributed to bridging the knowledge from sports towards rehabilitation and clinical practice. Through pilot studies the application of different implicit motor learning strategies to facilitate walking in people after stroke and people with Parkinson's were explored (chapters 2 and 3). Based on these studies the first randomised controlled trial to examine the effects of an implicit compared to explicit motor learning walking intervention to improve walking performance in people after stroke was developed, conducted and evaluated (chapters 4 to 6). The results provide new insights in the application, effects and feasibility of implicit motor learning within neurological rehabilitation. Furthermore, findings seem reflective for clinical practice as the trial took place within a real-life setting (the home environments of the patients), were focused on a common daily life task (that is walking) and a representative sample was included.

Additionally, this thesis innovative of nature as we re-designed and optimized an existing technology rather that developing a new product from scratch (which seems way more efficient). Everyday new (supportive) technologies get developed, however many of these new innovative systems or programs are not being used as regular as intended. One reason may be that often engineers develop a technology that functionally operates perfectly but is not attractive or inviting to use. Designing products and user interfaces as described within this thesis, may contribute to the creation of products that lead to a more meaningful user experience. 
154 | Valorisation 


\section{Acknowledgements / Dankwoord}

"Acknowledging the good that you already have in your life is the foundation for all abundance."

Eckhart Tolle 
Allereerst wil ik mijn promotor en copromotoren bedanken die mij tijdens dit bijzondere traject hebben begeleid: ik vond het ontzettend fijn om door jullie begeleid te worden.

Sandra, wat fijn dat jij mijn promotor was! Dank voor je begeleiding en steun tijdens deze, voor mij vier intensieve jaren van onderzoek doen. De introductie van de statafel tijdens de promotie overleggen was een welkome afwisseling tijdens het vele (zittend) schrijven in de laatste periode van mijn proefschrift. Jouw kritische vragen en onze discussies tijdens deze overleggen hielden mij scherp! Bedankt voor je fijne manier van begeleiden, ondanks een drukke planning was je altijd daar en kon ik altijd bij jou terecht.

Kenneth, via jou kwam ik terecht in een voor mij nieuwe onderzoeksgroep waar ik me al snel thuis voelde. Ik kwam (en kom) er heel graag. Bedankt voor je prettige manier van begeleiden. Nog steeds kom je altijd even checken hoe het met mij gaat en dat waardeer ik zeer. Het is ook ontzettend fijn hoe je ook nu meedenkt in mogelijke vervolgstappen (welke deze ook mogen zijn en waar deze dan ook naar toe zullen leiden) door je netwerk open te stellen, een luisterend oor te zijn en mee te denken. Bedankt!

Susy, inmiddels is het alweer acht jaar geleden dat ik jou als 3 e jaars fysiotherapie student tegenkwam. Ik wilde graag kennis maken met wetenschappelijk onderzoek en nog graag een keer naar het buitenland en jij gaf me de mogelijkheid om deze wens te vervullen. Ik had nooit gedacht dat mijn bachelor scriptie uiteindelijk zou leiden tot een Master opleiding in Exeter en vervolgens dit promotietraject. Het feit dat ik wist dat ik o.a. met jou zou mogen samenwerken in dit project, was een grote reden voor mij om op de functie van promovendus te solliciteren. Ontzettend bedankt voor alles wat ik van jou heb mogen leren, zowel op gebied van onderzoek, onderwijs maar ook als persoon. Ik heb genoten, en geniet nog steeds van al onze koffie's, walk \& talks. Nu ga ik langzaam maar zeker de volgende fase in als onderzoeker. Ik waardeer de vrijheid, mogelijkheden en steun die je mij biedt en geboden hebt enorm. Ik kijk uit naar nieuwe projecten en samenwerkingen die hopelijk nog zullen volgen. Bedankt voor alles!

De leden van de beoordelingscommissie, Prof. Rob Smeets, dr. Sil Aarts, Prof. Ton Lenssen, Dr. Elmar Kal en Prof. Bert Steenbergen wil ik hartelijk bedanken voor jullie tijd en expertise bij het beoordelen van mijn proefschrift. De corona bedank ik voor hun aanwezigheid en het lezen van mijn proefschrift. 
Dit onderzoek had nooit kunnen plaatsvinden zonder de financiering van Nationaal Regieorgaan Praktijkgericht Onderzoek SIA. Bedankt voor jullie vertrouwen en support in het project.

Ik wil graag alle deelnemers bedanken voor de moeite en tijd die ze hebben genomen om deel te nemen aan de studie. Zonder jullie had dit project uiteraard nooit kunnen plaatsvinden. Sommige van jullie hebben grote afstanden afgelegd om deel te nemen aan de metingen in het lab. Dank voor jullie flexibiliteit bij het plannen van de vele metingen en looptrainingen. Bedankt voor jullie interesse, deelname, en openheid!

Dit project had nooit kunnen plaatsvinden zonder de hulp van de volgende praktijken: ParaMedisch Centrum Zuid, Regtop Fysiotherapie, Sevagram, Fysiovision, Fysiotherapie Snijders, Houben Fysiotherapie, Praktijk voor fysiotherapie Augenbroe, Adelante Zorggroep, Zuyderland en het Maastricht UMC+ bedankt! In het bijzonder wil ik alle therapeuten van Adelante Zorggroep, Regtop, Praktijk voor fysiotherapie Augenbroe en Sevagram bedanken voor het meedenken en evalueren van alle kennis rondom de toepassing van impliciet motorisch leren en het sensoren-feedback systeem Stappy in de klinische praktijk.

Melanie, dit onderzoek was nooit zo gelopen als jij er niet was geweest. Wat ontzettend fijn dat ik dit traject samen met jou mocht doorlopen, ik had me geen betere sparrings- en onderzoekspartner kunnen bedenken. Ook van jou heb ik ontzettend veel mogen leren. Ik geniet ook nu nog van onze fijne samenwerking, interessante discussies en ideeën over motorisch leren. Intussen zijn we samen aan nieuwe vervolgprojecten begonnen en ik ben erg benieuwd naar wat voor nieuwe projecten of samenwerkingen er nog zullen volgen. Tijdens jouw publieke verdediging mocht ik als paranimf al aan jou zijde staan, wat was ik trots! $\mathrm{Nu}$ is de tijd daar om zelf als promovendus mijn proefschrift te verdedigen. Bedankt voor alles wat je mij hebt meegegeven.

Mark, it was 2013 when Floor and I first knocked on your door. You made us feel welcome in Exeter from the beginning. Since then, you have been guiding me into the world of research and motor learning. I have really appreciated your support and advice throughout the years. Thank you for being there and I hope that we will continue to collaborate in future projects. Nic, Annika and Liv thank you for always being so open and welcoming. You're always welcome in Maastricht!

Vicki, thank you so much for your support during my first steps in research. I'm very thankful for your supervision during my time in Exeter. From guiding me through my dissertations to supporting me during my first (big) presentation! I hope that we may collaborate again in the future. 
Floor, samen met jou ben ik op de opleiding fysiotherapie tijdens onze bachelor thesis dit avontuur begonnen! Beide zijn wij een andere weg in gegaan, ik verder als onderzoeker en jij verder als fysio en later als Physician Assistant. We zien elkaar niet vaak, maar als we elkaar zien dan is het altijd weer als vanouds. Bedankt voor het Exeter-avontuur dat we samen mochten ervaren.

Lieve Nadine, zonder jou waren deze afgelopen vier jaar heel anders geweest. Wat ben ik blij met jou als vriendin. Met jou kan ik alles in vertrouwen delen, doen, en laten gaan. Bedankt voor alle gezelligheid, steun, verhalen, etentjes, festivals en nog veel meer. Ik kijk uit naar alles wat nog zal komen en hoe onze wegen zullen lopen. Ik ben trots op jou! Het is een eer dat jij mijn paranimf wilt zijn tijdens mijn openbare verdediging.

Lieve Kyra, van de een op de andere dag kon jij inspringen bij de metingen in het lab. Inmiddels hebben wij er heel wat uren op zitten, zowel binnen als buiten het lab. Samen hebben we dan ook al veel avonturen meegemaakt en ik ben benieuwd naar welke andere avonturen er nog zullen volgen (ook veel verjaardagen gevierd, haha). Congressen samen met jou zal ik niet snel vergeten. Bedankt voor alles en wat ontzettend gaaf dat jij mijn paranimf wilt zijn.

De academie fysiotherapie van Zuyd Hogeschool, in het bijzonder Monique van den Broek en Claudy Cobben bedankt voor de mogelijkheden en support. Graag bedank ik mijn collega's van de opleidingen fysiotherapie, ergotherapie, biometrie, logopedie, communication and multimedia design voor jullie (emotionele) support en bijdrage op verschillende vlakken, zoals jullie hulp in het bewegingslab, de gezamenlijke begeleiding van afstudeerprojecten, het integreren van kennis over motorisch leren binnen het onderwijs, het programmeren van projecties en nog veel meer. Wat ontzettend gaaf dat de kennis van dit project nu al terugvloeit naar het onderwijs!

Mijn lieve collega's van de cluster lectoraten, bedankt voor jullie interesse en steun deze vier jaren! In het bijzonder wil ik ook mijn collega's van het lectoraat Voeding, Leefstijl en Bewegen bedanken, wat een prachtig en bijzonder team is dit. Bedankt voor al jullie support, enthousiasme, tips en steun. Ruth en Steffy, bedankt voor jullie tips en adviezen bij het maken van materiaal voor mensen met afasie. Gaston, wat gaaf dat we samen een artikel hebben mogen schrijven. Bedankt voor je creativiteit, enthousiasme en de leuke samenwerking! Renée, ontzettend bedankt voor je ondersteuning op vele vlakken, vooral met Stappy hebben we veel samengedaan. Albine en Esther Stoffers bedankt voor jullie kennis en ervaringen omtrent cliëntparticipatie! Mijn collega's van de voormalige promovendi kamer (D1.230) bedanken. Steffy, Stephie, Jerome, Kim, Jolanda, Darcy, Kyra, Ruth, Esther, Iris en Hester het was heel fijn om al onze ervaringen met elkaar te kunnen delen. Ik mis de kamer! Bedankt voor jullie luisterend oor, support, en adviezen. 
Graag bedank ik Bart en Pieter, van de AV-dienst op Zuyd hogeschool. Het maken en creëren audiovisueel materiaal is van onschatbare waarde voor de vertaling van kennis naar het onderwijs en de praktijk. Bedankt voor jullie ondersteuning bij het ontwikkelen van deze materialen!

Stephanie, Marja, Jacqueline en Prisca, jullie waren betrokken en onmisbaar bij zo veel verschillende taken zoals het organiseren van symposia, plannen van promotie overleggen, nalezen/nakijken van teksten en nog veel meer. Bedankt alle ondersteuning bij alle verschillende activiteiten!

Lieve collega's en (ex)medepromovendi van de vakgroep Voeding en Bewegingswetenschappen in Maastricht. Bedankt voor het warme welkom binnen de vakgroep. A special thanks to my former and current roommates (room G2.220): Pieter, Bernard, Hans, Chris, HQ, Yingyi, and Bas, thank you so much for the support, talks, coffees, nerdy jokes, fun, joy and much more. It has been great to share the room, our experiences, ups and downs together and my $\mathrm{PhD}$ would not have the same without you guys! Wai-Yan I am still impressed by your fast ability to find Wally! We zaten in andere kamers maar heb even goed genoten van jouw gezelligheid! Irene en Kyra, wat super om jullie om me heen te hebben, heerlijk om af en toe gewoon wat 'girl' talk tussen alle mannen op de afdeling te hebben. Irene, ik ben blij dat ik tenminste één iemand van de afdeling aan het squashen heb gekregen! Harry, Desiree, en Yolanda, bedankt voor jullie ondersteuning op allerlei vlakken zoals het plannen van promotie overleggen, installeren van software of reserveren van ruimtes.

Through conferences and collogues I've met so many new people these years. Especially I would like to thank Maud, Pieter (Universiteit Hasselt), Tom (Universiteit Groningen), Liis (University of Waikato), Elmar, and Toby (Brunell University London). Thanks for the interesting conversations, and great social events. I'm looking forward to future conferences and potential new collaborations together.

Rich Masters, it was during my bachelor thesis that I came in contact with you and I was so happy that you gave feedback. I got to know you better when you visited the Netherlands in 2014. We got stuck in traffic on our way to the airport: I can still picture the trip: no trains, a (chaotic) crowd waiting to enter the bus and a lot of luggage (including your golf clubs) but we managed to make it on time to our flights to Exeter. Thank you for your openness, enthusiasm and support during the past years. I hope we can collaborate in future projects and I am curious to see how things will develop. Liis Uiga, it was great meeting you at ISPGR and I hope to visit you one day in either New Zealand and/or Estonia. It would be great if we could do some work and spend some time together in the future! 


\section{Dit project had onmogelijk kunnen plaatsen vinden zonder de hulp en steun van de}

volgende personen.

Michael Nunns, thank you for teaching me how to use the CODA-motion analyses system, and for writing the Matlab script to analyse the data. Wouter Bijnens, dank voor jouw expertise en hulp met het 3D VICON-motion analyses systeem, de installatie van de projector, en het schrijven van de Matlab scripts. Toine Diederen en Dennis Odekerken bedankt voor jullie expertise en hulp bij de planning van alle metingen bij het motion lab. Ryan Wilms, dank voor het programmeren van de projecties en het bijwonen van de eerste metingen waarbij dit systeem werd gebruikt. Peter Konsten, bedankt voor de ondersteuning bij het ontwikkelen van de online vragenlijsten en je altijd snelle reacties op vragen of aanpassingen.

Anja Minheere, Else de Bont en Nathalie Sieben; jullie deelname als ervaringsdeskundige binnen dit project was ontzettend betekenisvol. Ik heb veel van jullie mogen leren, bedankt voor jullie inzet, adviezen, maar ook jullie enthousiasme tijdens dit project. Nu mogen wij onze samenwerking in een vervolgproject nog even voortzetten en wie weet welke andere projecten nog zullen volgen. Bedankt voor alles!

Beste Jos Halfens, Jos Kurvers, en Wiel Regtop, wat een eer dat jullie als therapeuten en experts op gebied van neuro-revalidatie wilden deelnemen aan dit project. De kennis en praktische ervaringen die jullie meebrachten waren een waardevolle en welkome toevoeging. Het plannen van alle looptrainingen was een uitdaging. Mijn dank is dan ook groot voor jullie enorme flexibiliteit en inzetbaarheid bij plannen van deze sessies! Ik heb ontzettend genoten van de samenwerking met jullie.

Monique Rothbauer, Inge de Lang, Joep Westbroek en Maartje Feuler, wat ontzettend fijn en gaaf dat jullie naast jullie werk in de praktijk ook wilde meedenken en helpen binnen dit onderzoeksproject. Jullie hebben ontzettend veel werk verricht en heel Limburg afgereisd. Bedankt voor jullie inzet en flexibiliteit. Het was ontzettend fijn om dit traject samen met jullie te doorlopen.

Ritch te Kampe en Kyra Theunissen, jullie waren multi-inzetbaar, wat een berg werk hebben jullie verricht! Bedankt voor al jullie hulp en flexibiliteit, maar daarnaast ook dank voor de leuke, gezellige tijd die ik met jullie samen heb beleefd tijdens al deze activiteiten. Het vele werk heeft jullie niet afgeschrikt want, jullie zijn nu beide je eigen $\mathrm{PhD}$ en onderzoekstraject gestart. Ik kijk uit naar jullie eerste resultaten en alles wat nog zal volgen. 
Evelien Wouters, Marianne Nieboer en Lydia Willemse, er zijn heel wat kilometers richting het uiterste zuiden van het land gereisd o.a. voor de vele huisbezoeken om het gebruik van het sensoren-feedback systeem Stappy bij de deelnemers thuis te evalueren. Bedankt voor jullie waardevolle feedback, expertise en de samenwerking!

Dear Elisabetta, and Bojan, it's been a pleasure to have worked with you on the sensorfeedback system Stappy. I really enjoyed our collaboration. Thank you for the always fast and clear responses to our questions. Also, thanks for the great time and talks we had in Trento (Italy) while I was visiting you there with Ritch and Kate.

Sanne Kusters, van Burgerkracht Limburg. Bedankt voor het bemiddelen in het vinden van de ervaringsdeskundige en het werven van cliëntvertegenwoordigers.

Anneke Hiemstra en Hans Leutscher namens Kenniscentrum Sport. Bedankt voor jullie ondersteuning bij de disseminatie van de onderzoeksresultaten binnen dit project!

Ryan Wilms, Kate Smit, Marloes Jansen, Annika Ries, en Marius Wientgen dank voor het vele werk dat jullie hebben verzet op verschillende vlakken zoals jullie ondersteuningen bij de dataverzameling, dataverwerking, maar ook jullie expertise zoals op het gebied van userinterface design of het programmeren van projecties.

Dear Chris McCrum, Chris Woolnough, Cate Stubbe and Kirsty Brock, thank you so much for helping me improving the English language of manuscripts and chapters. I really appreciate your openness and willingness (often on a short notice) to help. Thank you for everything!

Ik wil graag alle studenten bedanken die via hun afstudeerprojecten betrokken waren in bij dit project. Kasper Bongaerts, Jur Jorissen, Dominic Bednas, Samantha Stammen, Robbin Steijaert, Patrick Vermulst, Anniek Janssen, Eva Maessen, Mariëlle Peters, Innez Robbertz, Anne Krekels, Jeroen de Ruijter, Cock Hohmbergh, Stijn Houben, Jari Dohmen, Mirthe Cox, Fenne Loots, Jenny Schillings, Shanna Smeijsters, Laura de Brouwer, Femke Verheijen, Liam Bewersdorf, Sanne Mullenders, Myrthe Rutten, Kate Smit, Fé Rietjens, Lisa Klein, Ritch te Kampe, en Robin Tiberius bedankt!

Mijn schoolcarrière was bijzonder en ik heb niet de standaard route genomen om te komen waar ik nu ben. Ik ben een aantal personen bijzonder dankbaar voor hun vertrouwen in mij. Roel Scholten, Andre Kabo, (basisschool de Kring), Huub Kamps, Paul Mes (Trajectum college), Helma Baeten, en Nicole Hoedemakers (Sint-maartenscollege) bedankt voor de kansen en het vertrouwen die jullie mij hebben gegeven. Karin, een heel jaar wiskunde in één zomer inhalen (van VMBO naar HAVO) is niet niks. Bedankt voor al je hulp, bijlessen en support tijdens deze intensieve jaren! 
Mark en Liesbeth, wat gaaf dat ik de afgelopen 3,5 jaar samen met jullie als collega's heb mogen werken op de squashbanen! Bedankt voor alles wat ik van jullie heb geleerd op en buiten de squashbanen. Maike, Carolien, Irene, I am really glad that you participated in one of my squash courses and I am even happier that this resulted in our friendship. Thanks for the fun times next to my busy $\mathrm{PhD}$ life.

Mijn lieve vrienden van het squashteam 'Dagblad de Limburger' Joos, Benti, Jerry, Adrian, Jacques, Stephan, Albert maar ook zeker Petran, Berenice, en Veronique. Wat een warm, gezellig, en enthousiast team! Bedankt voor de gezellige, sportieve avonden en zo nu en dan de soms wat vroegere zondagochtenden op de squashbanen!

When I started my Masters degree I could never have imagined what a special year this would become. Also in the years that followed until now we've always been in touch, which means a lot to me. To my dear friends, Paola, Chiara, Kirsty, Lucy, Susannah, and Nicky. I am very grateful for the times we spent together in and around Exeter and it has been one of my best years so far. I am very happy that, even now 5 years later, we still see each other. Thanks for the many adventures we had together such as the nice summer trip to Cornwall, the Ecuadorian adventure with Paola, Lucy's wedding, the days in Kent, Dartmoor, Plymouth, London etc. Can't wait to begin new trips and adventures with you girls. Mama Li.

Wat heb ik een prachtige familie. Lieve Simba-jot, Oma Isa, A-Ghén, Jenny, Peter, Oma Gien, Opa Bakkebaard, Shirley, Lydia, Rob, Willy, Lillian, Jim, Luc, Sem, en Pascalle bedankt voor al jullie support, steun, en gezelligheid. Ik hou van jullie. Lieve Karin en Cate, jullie zijn er altijd voor mij. Bedankt voor alle sportieve, studie- en ontspanningsactiviteiten. Bedankt voor jullie liefde. Dear Monica, and Jean-Piere, it was a pleasure meeting you and I had such a great time. Looking forward to many new adventures to come. My house is always open to you. Sabine I look forward to meeting you.

Lieve mama, papa, Paul, Lisa, en Lion, zonder jullie liefde, aandacht en zorgen had dit traject er natuurlijk heel anders uit gezien. Door de vele verschillende scholen was het niet altijd even makkelijk, ik moest mijn weg vinden en school was ook niet helemaal mijn ding. Door jullie liefde ben ik gekomen waar ik nu ben en wil zijn. Ik ben jullie ontzettend dankbaar voor alles wat jullie mij hebben meegegeven. Ik hou van jullie!

Brassa,

Li-Juan

$162 \mid$ Acknowledgements 


\section{Curriculum Vitae}




\section{Publications within this thesis}

Jie LJ, Jamin G, Smit K, Beurskens A, Braun S. Design of the user interface for "Stappy", a sensor-feedback system to facilitate walking in people after stroke: a user-centred approach. Disability and Rehabilitation: Assistive Technology. 2019;0(0):1-9.

Jie LJ, Kleynen M, Beurskens A, Meijer K, Braun S. The Effects of Implicit and Explicit Motor Learning in Gait Rehabilitation of People After Stroke: Protocol for a Randomized Controlled Trial. JMIR Res Protoc. 2018;7(5):e142-e142.

Kleynen M, Jie LJ, Theunissen K, Rasquin SM, Masters RS, Meijer K, et al. The immediate influence of implicit motor learning strategies on spatiotemporal gait parameters in stroke patients: a randomized within-subjects design. Clin Rehabil. 2019;33(4):619-630.

Jie LJ, Goodwin V, Kleynen M, Braun S, Nunns M, Wilson M. Analogy learning in Parkinson's disease: A proof-of-concept study. Int J Ther Rehabil. 2016;23(3):123-130.

\section{Submitted}

Jie LJ, Kleynen M, Meijer K, Beurskens A, Braun S. Implicit and explicit motor learning interventions for gait in people after stroke: a process evaluation on fidelity and feasibility performed alongside a randomized controlled trial. Preprint at medRxiv doi: 10.1101/2020.01.17.20017897. 2020

Jie LJ, Kleynen M, Beurskens A, Meijer K, Braun S. Implicit and explicit motor learning interventions have similar effects on walking speed in people after stroke: a randomized controlled single blind trial. Preprint at medRxiv doi: 01101/19008797. 2019.

\section{Other publications}

McCrum C, Essers J, Jie LJ, Liu W-Y, Meijer K. Commentary: Older adults can improve compensatory stepping with repeated postural perturbations. Front Aging Neurosci. 2016;8:111.

Kleynen M, Wilson MR, Jie LJ, te Lintel Hekkert F, Goodwin VA, Braun SM. Exploring the utility of analogies in motor learning after stroke: a feasibility study. Int J Rehabil Res. 2014;37(3):277-280. 


\section{National Journals}

Jie LJ, van den Heuvel R, Braun SM, Kleynen M. Looptraining met behulp van technologie; ervaringen van CVA-patiënten en therapeuten met het sensoren-feedback systeem 'Stappy'. Keypoint Tijdschrift voor behandelaars in de neurorevalidatie. 2020;43E(3):16-19.

Jie LJ, Jie A, Hoff J, Tissingh G. Telemonitoring verbetert parkinsonzorg. Medisch Contact. 2016(14):21-23.

\section{Conference Proceedings}

Jie LJ [invited speaker] The power of implicit motor learning. (Oral presentation at the symposium Human balance across the lifespan, Maastricht, the Netherlands, December 2019).

Jie LJ, Kleynen M, Meijer K, Beurskens AJ, Braun SM. Implicit and explicit motor learning in gait rehabilitation of people after stroke: A randomized controlled single blind trial. (Poster presentation at the Dag van de Fysiotherapeut, Den Bosch, the Netherlands, November 2019).

Jie LJ [invited speaker] Nooit meer expliciet? (Oral presentation at the Afscheidsreceptie Frank van Hartingsveld, Hogeschool van Amsterdam, Amsterdam, the Netherlands, October 2019).

Jie LJ [invited speaker] Looptraining bij mensen na een beroerte: de toepassing van impliciet motorisch leren en het gebruik van een sensoren feedback systeem. (Oral presenation at the Keypoint Lustrumcongres, Fort Voordrop, the Netherlands, October 2019).

Jie LJ, Braun S, Kleynen M, Theunissen K, Rasquin S, Masters R, Meijer K, Beurskens A, Braun S. The immediate influence of implicit motor learning on gait in stroke patients. (Poster presentation at the European Society of Analysis in Adults and Childres (ESMAC) Annual Meeting, Amsterdam, the Netherlands) Published in Gait and Posture, doi: https://doi.org/10.1016/j.gaitpost.2019.07.148

Jie LJ, Jamin G, Smit K, Beurskens A, Braun S. Design of the user interface for "Stappy”, a sensor-feedback system to facilitate walking in people after stroke: a user-centered approach. (Poster presentation at the European Society of Analysis in Adults and Childres (ESMAC) Annual Meeting, Amsterdam, the Netherlands). Published in Gait and Posture, doi: https://doi.org/10.1016/j.gaitpost.2019.07.149 
Jie LJ, Kleynen M, Meijer K, Beurskens AJ, Braun SM. Implicit and explicit motor learning in gait rehabilitation of people after stroke: A randomized controlled single blind trial. (Poster presentation at the International Society of Poster and Gait Research World Congress, Edinburgh, Scotland, July 2019).

Jie LJ [invited speaker] Impliciet motorisch leren met behulp van analogieën. (Oral presentation at the focused symposium "Motorisch leren in de neuro-revalidatie", Wijk aan Zee, the Netherlands, February 2019).

Kleynen M, Jie LJ, Theunissen K, Rasquin S, Masters RS, Meijer K, Beurskens A, Braun SM. The immediate influence of implicit motor learning on gait in stroke patients. (Poster presentation at the 6th international RehabMove Congress, Groningen, the Netherlands, December 2018).

Jie LJ. Implicit motor learning to improve gait in people after stroke. How do you do this? (Oral presentation at the Symposium Motor learning in sport and rehabilitation: What can we learn from each other?, Maastricht, Netherlands, October 2018).

Jie LJ, Kleynen M. The power of implicit motor learning. (Oral presentation at the inauguration of dr. S. Braun., dr. E. van Rossum and dr. R. Daniëls, Maastricht, Netherlands, March 2018).

Jie LJ. As easy as a walk on the beach - using analogies to promotor motor relearning. (Oral presentation at the mini-symposium "Putting motor learning into (neurorehabilitation) practice: A focus on analogies, cueing, and external focus of attention" at the Dutch Congress of Rehabilitation Medicine, Maastricht, Netherlands, November 2016).

Jie LJ, Kleynen M, Meijer K, Rasquin SM, Masters RS, Beurskens AJ, Braun SM. The power of implicit motor learning: a project overview. (Poster Presentation at the Annual NUTRIM symposium, Maastricht, the Netherlands, December 2019)

Jie LJ, Kleynen M, Meijer K, Rasquin SM, Masters RS, Beurskens AJ, Braun SM. The power of implicit motor learning: a project overview. (Poster presentation at Bijzondere BijZOandeR, Hoensbroek, the Netherlands, December 2019).

Jie LJ, Goodwin V, Kleynen M, Braun SM, Nunns M, Wilson M. Analogy learning in Parkinson's; As easy as a walk on the beach: A proof-of-concept study. (Poster presentation at the Society for Movement Analysis Laboratories in the Low Lands (SMALLL) Annual Congress, Maastricht, the Netherlands, November 2015). 
Jie LJ, Kleynen M, Meijer K, Rasquin SM, Masters RS, Beurskens AJ, Braun SM. The power of implicit motor learning: a project overview. (Poster presentation at Kennis In Bedrijf, November 2015, Heerlen, the Netherlands).

Jie LJ, Jie GKS, Tissingh G, MijnParkinsoncoach, telemonitoring en e-consulten bij Parkinonpatienten, (Oral and Poster presentation at the Symposium "Innovatie in de zorg", Sittard-Geleen, the Netherlands, June 2015).

Jie LJ, Wilson MW, Goodwin V, Analogy learning in walking in people with Parkinson's Disease (Oral presentation at the symposium "Motor learning in different populations within neurorehabilitation: bridging the gap between research and practice" at the Congress on Neurorehabilitation and Neural Repair, Maastricht, Netherlands, May 2015)

Jie, LJ., M, Wilson, Goodwin, V., Nunns, M., M, Kleynen \& Braun, S. M. Analogies and stride length regulation in Parkinson's. (Oral presentation at the Health and Medical Research showcase, Exeter, United Kingdom, June, 2014).

Jie LJ, Wilson MW, Goodwin V, The utility of analogy learning. (Oral presentation at the symposium “Wat u beweegt!", Heerlen, Netherlands, May, 2014)

Kleynen M, Wilson MW, Jie LJ., Hekkert FTL, Goodwin V, \& Braun SM. 'Exploring the utility of analogies in motor learning after stroke: A feasibility study', (Poster presentation at the World Congress for Neurorehabilitation, Turkey, April 2014).

Jie, LJ, Linström, M. IP-MAND: A challenge for students and teachers. (Oral and poster presentation at the Physiotherapy Education Congress Vienna, Austria, November 2012). 
168 | Curriculum Vitae 


\section{About the Author}

Li-Juan Jie was born on the 13th of July 1991 in Lanaken, Belgium. In 2009, she graduated from secondary school at the Sint-Maartenscollege Maastricht (the Netherlands). She then studied Physiotherapy at Zuyd University of Applied Sciences in Heerlen, the Netherlands. In her senior year she went to Exeter (the United Kingdom), for a research (University of Exeter) and physiotherapy (Newton Abbot Community Hospital) internship. During here time in Exeter she also actively participated in the Action After Stroke group (exercise group for people after stroke) and completed a module Sport Psychology at the University of Exeter. After

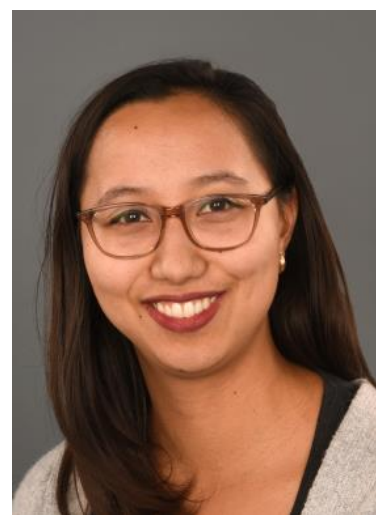
obtaining her Bachelor's degree in Physiotherapy in 2013, she received a scholarship from the VSB foundation to start a Master's degree in Sport and Health sciences at the University of Exeter, where she graduated in 2014. From 2012 to 2015 she also worked as a student researcher within Research Centre for Autonomy and Participation of Persons with a Chronic Illness. In 2015 she started working as a teacher at the physiotherapy department at Zuyd University of applied sciences.

It was during her Bachelor and Master thesis that she became interested in the topic motor learning and especially the applicability of implicit motor learning in neurological rehabilitation. During her bachelor and master theses she explored whether and how implicit motor learning could be applied to improve walking performance in people after Stroke and people with Parkinson's. After obtaining her Master's degree she started her $\mathrm{PhD}$ project in 2015 and continued her work on the application of implicit motor learning in neurological rehabilitation under supervision of Prof. dr. Sandra Beurskens, dr. Susy Braun and dr. Kenneth Meijer. The PhD project was embedded within the department of Family Medicine (CAPHRI), the department of Nutrition and Movement Sciences (NUTRIM) at Maastricht University and the Research Center for Nutrition, Lifestyle and Exercise (Academy of Physiotherapy) at Zuyd University of Applied Sciences. Her PhD was part of a larger project "The Power of Implicit Motor Learning 2.0" that recently was awarded with the public price and the 2nd price from the professional jury at the RAAKaward 2019 (from the SIA foundation for best applied research in the Netherlands). Furthermore, Li-Juan recently received the YERUN (Young European Research Universities) Mobility Award that she will use to exchange knowledge and strengthen the network with Brunell University London. Currently, she works as a teacher and researcher at the Research Center for Nutrition, Lifestyle and Exercise (Zuyd University of Applied Sciences). She hopes to continue her research on the topic of implicit motor learning and make the generated knowledge available and understandable for clinical and daily life practice. 
170 | 


\section{REFERENCES}

1. Arnold SJ. Morphology, performance and fitness. Integr Comp Biol 1983.

2. Reber AS. The cognitive unconscious: An evolutionary perspective. Conscious Cogn. 1992;1(2):93-133.

3. Masters RSW, Poolton JM, Maxwell JP. Stable implicit motor processes despite aerobic locomotor fatigue. Conscious Cogn. 2008;17(1):335-338.

4. Kleynen M, Braun SM, Rasquin SMC, Bleijlevens MHC, Lexis MAS, Halfens $\mathrm{J}$, et al. Multidisciplinary Views on Applying Explicit and Implicit Motor Learning in Practice: An International Survey. PloS One. 2015;10(8):e0135522e0135522.

5. Fitts PM, Posner MI. Learning and skilled performance in human performance. Belmont, CA: Brock-Cole. 1967.

6. Johnson L, Burridge JH, Demain SH. Internal and external focus of attention during gait re-education: an observational study of physical therapist practice in stroke rehabilitation. Phys Ther. 2013;93(7):957-966.

7. Baddeley AD, Hitch GJ. Working Memory. Vol 8C: New York: Academic Press; 1974.

8. Masters RSW, Poolton J. Advances in implicit motor learning. In: Hodges A.M N, Williams, eds. 2nd ed.: Routledge; 2012:59-75.

9. Dienes Z, Berry D. Implicit learning: Below the subjective threshold. Psychon Bull Rev. 1997;4(1):3-23.

10. Lam WK, Maxwell JP, Masters RS. Analogy versus explicit learning of a modified basketball shooting task: performance and kinematic outcomes. $J$ Sports Sci. 2009;27(2):179-191.

11. Liao CM, Masters RS. Analogy learning: a means to implicit motor learning. $J$ Sports Sci. 2001;19(5):307-319.

12. Masters RSW. Knowledge, knerves and know-how: The role of explicit versus implicit knowledge in the breakdown of a complex motor skill under pressure. Brit J Psychol. 1992;83(3):343-358.

13. Hardy L, Mullen R, Jones G. Knowledge and conscious control of motor actions under stress. Br J Psychol. 1996;87 ( Pt 4)(4):621-636.

14. Lam WK, Maxwell JP, Masters R. Analogy learning and the performance of motor skills under pressure. J Sport Exerc Psychol. 2009;31(3):337-357.

15. Duijn Tv. The Influence of Analogy Instructions on Motor Skills: Processing, Preference and Performance 2018.

16. Maxwell JP, Masters RSW, Eves FF. From novice to no know-how: A longitudinal study of implicit motor learning. J Sports Sci. 2000;18(2):111-120.

17. Reber AS. Implicit learning of artificial grammars. J Verbal Learning Verbal Behav. 1967;6(6):855-863.

18. Nissen MJ, Bullemer P. Attentional requirements of learning: Evidence from performance measures. Cogn Psych. 1987.

19. Capio CM, Poolton JM, Sit CHP, Eguia KF, Masters RSW. Reduction of errors during practice facilitates fundamental movement skill learning in children with intellectual disabilities. J Intellect Disabil Res. 2013.

20. Masters RSW, Lo CY, Maxwell JP, Patil NG. Implicit motor learning in surgery: Implications for multi-tasking. Surgery. 2008. 
21. Zhu FF, Poolton JM, Wilson MR, Hu Y, Maxwell JP, Masters RSW. Implicit motor learning promotes neural efficiency during laparoscopy. Surg Endosc. 2011.

22. Tse CY, Wong A, Whitehill T, Ma E, Masters R. Examining the cognitive demands of analogy instructions compared to explicit instructions. Int J Speech Lang Pathol. 2016;18(5):465-472.

23. Boyd LA, Winstein CJ. Explicit information interferes with implicit motor learning of both continuous and discrete movement tasks after stroke. Journal of neurologic physical therapy: JNPT. 2006;30(2):46-57.

24. Kleynen M, Wilson MR, Jie LJ, te Lintel Hekkert F, Goodwin VA, Braun SM. Exploring the utility of analogies in motor learning after stroke: a feasibility study. Int J Rehabil Res. 2014;37(3):277-280.

25. Orrell AJ, Eves FF, Masters RS. Motor learning of a dynamic balancing task after stroke: implicit implications for stroke rehabilitation. Phys Ther. 2006;86(3):369-380.

26. Poolton JM, Masters RSW, Maxwell JP. Passing thoughts on the evolutionary stability of implicit motor behaviour: Performance retention under physiological fatigue. Conscious Cogn. 2007;16(2):456-468.

27. Kleynen M, Braun SM, Bleijlevens MH, Lexis MA, Rasquin SM, Halfens J, et al. Using a Delphi technique to seek consensus regarding definitions, descriptions and classification of terms related to implicit and explicit forms of motor learning. PloS One. 2014;9(6):e100227-e100227.

28. Tatemichi TK, Desmond DW, Stern Y, Paik M, Sano M, Bagiella E. Cognitive impairment after stroke: frequency, patterns, and relationship to functional abilities. J Neurol Neurosurg Psychiatry. 1994;57(2):202-207.

29. Boyd LA, Quaney BM, Pohl PS, Winstein CJ. Learning implicitly: Effects of task and severity after stroke. Neurorehabilitation and Neural Repair. 2007;21(5):444-454.

30. Craig P, Dieppe P, Macintyre S, Michie S, Nazareth I, Petticrew M. Developing and evaluating complex interventions: the new Medical Research Council guidance. Int J Nurs Stud. 2013;50(5):587-592.

31. Langhorne P, Bernhardt J, Kwakkel G. Stroke rehabilitation. Lancet. 2011;377(9778):1693-1702.

32. Sluijs EM, Kok GJ, Van der Zee J. Correlates of exercise compliance in physical therapy. Phys Ther. 1993;73(11):771-782.

33. Putrino D. Telerehabilitation and emerging virtual reality approaches to stroke rehabilitation. In:2014.

34. Casamassima F, Ferrari A, Milosevic B, Ginis P, Farella E, Rocchi L. A wearable system for gait training in subjects with Parkinson's disease. Sensors (Basel). 2014;14(4):6229-6246.

35. Peek ST, Wouters EJ, van Hoof J, Luijkx KG, Boeije HR, Vrijhoef HJ. Factors influencing acceptance of technology for aging in place: a systematic review. Int J Med Inform. 2014;83(4):235-248.

36. Jankovic J. Parkinson's disease: clinical features and diagnosis. J Neurol Neurosurg Psychiatry Res. 2008;79(4):368-376.

37. Morris ME, Matyas TA, Iansek R, Summers JJ. Temporal stability of gait in Parkinson's disease. Phys Ther. 1996;76(7):763-777. 
38. Morris ME, Iansek R, Matyas TA, Summers JJ. Ability to modulate walking cadence remains intact in Parkinson's disease. Journal of neurology, neurosurgery, and psychiatry. 1994;57(12):1532-1534.

39. Meireles J, Massano J. Cognitive impairment and dementia in Parkinson's disease: clinical features, diagnosis, and management. Frontiers in neurology. 2012;3:88.

40. Rochester L, Hetherington V, Jones D, Nieuwboer A, Willems AM, Kwakkel $\mathrm{G}$, et al. Attending to the task: interference effects of functional tasks on walking in Parkinson's disease and the roles of cognition, depression, fatigue, and balance. Arch Phys Med Rehabil. 2004;85(10):1578-1585.

41. Allcock LM, Rowan EN, Steen IN, Wesnes K, Kenny RA, Burn DJ. Impaired attention predicts falling in Parkinson's disease. Parkinsonism \& related disorders. 2009;15(2):110-115.

42. Bloem BR, Hausdorff JM, Visser JE, Giladi N. Falls and freezing of gait in Parkinson's disease: a review of two interconnected, episodic phenomena. Movement disorders : official journal of the Movement Disorder Society. 2004;19(8):871-884.

43. Keus S, Munneke M, Graziano M, Paltamaa J, Pelosin E, Domingos J, et al. European Physiotherapy Guideline for Parkinson's Disease. http:// www.fizioterapeitiem.lv/attachments/article/307/4_eu_ guideline_parkinson_201412-development.pdf. Published 2014. Accessed 23 February 2016.

44. Keus SH, Bloem BR, Hendriks EJ, Bredero-Cohen AB, Munneke M, Practice Recommendations Development G. Evidence-based analysis of physical therapy in Parkinson's disease with recommendations for practice and research. Movement disorders : official journal of the Movement Disorder Society. 2007;22(4):451-460; quiz 600.

45. Morris ME. Movement disorders in people with Parkinson disease: a model for physical therapy. Phys Ther. 2000;80(6):578-597.

46. Lohnes CA, Earhart GM. The impact of attentional, auditory, and combined cues on walking during single and cognitive dual tasks in Parkinson disease. Gait Posture. 2011;33(3):478-483.

47. Kelly VE, Eusterbrock AJ, Shumway-Cook A. A review of dual-task walking deficits in people with Parkinson's disease: motor and cognitive contributions, mechanisms, and clinical implications. Parkinson's disease. 2012;2012:918719.

48. Claxton G, Vincer C. Mind, Hare Brain Tortoise: Why intelligence increases when you think less. Fourth Estate, London. 1998.

49. Reber AS. An evolutionary context for the cognitive unconscious. Philos Psychol. 1992;5(1):33-51.

50. Steenbergen B, van der Kamp J, Verneau M, Jongbloed-Pereboom M, Masters RS. Implicit and explicit learning: applications from basic research to sports for individuals with impaired movement dynamics. Disability and rehabilitation. 2010;32(18):1509-1516.

51. Masters RSW, Liao CM. Chunking as a characteristic of implicit motor learning. In: Stelter R, Roessler KK, eds. New Approaches to Sport and Exercise Psychology: Theories, Methods and Applications. Denmark, University of Copenhagen: Meyer \& Meyer; 2003:137-138.

52. Chase WG, Simon HA. Perception in chess. Cogn Psychol. 1973;4(1):55-81. 
53. Bond JM, Morris M. Goal-directed secondary motor tasks: their effects on gait in subjects with Parkinson disease. Arch Phys Med Rehabil. 2000;81(1):110116.

54. Brown LA, de Bruin N, Doan JB, Suchowersky O, Hu B. Novel challenges to gait in Parkinson's disease: the effect of concurrent music in single- and dualtask contexts. Arch Phys Med Rehabil. 2009;90(9):1578-1583.

55. Kiernan D, Walsh M, O'sullivan R, Fitzgerald D, O'brien T. Reliability of the CODA cx1 motion analyser for 3-dimensional gait analysis. Gait \& posture. 2014(39):S99-S100.

56. Doumas M, Smolders C, Krampe RT. Task prioritization in aging: effects of sensory information on concurrent posture and memory performance. Exp Brain Res. 2008;187(2):275.

57. Brach JS, Perera S, Studenski S, Katz M, Hall C, Verghese J. Meaningful change in measures of gait variability in older adults. Gait Posture. 2010;31(2):175-179.

58. Hass CJ, Bishop M, Moscovich M, Stegemoller EL, Skinner J, Malaty IA, et al. Defining the clinically meaningful difference in gait speed in persons with Parkinson disease. Journal of neurologic physical therapy : JNPT. 2014;38(4):233-238.

59. Baker K, Rochester L, Nieuwboer A. The immediate effect of attentional, auditory, and a combined cue strategy on gait during single and dual tasks in Parkinson,Äôs disease. Arch Phys Med Rehabil. 2007;88(12):1593-1600.

60. Ren J, Wu YD, Chan JS, Yan JH. Cognitive aging affects motor performance and learning. Geriatr Gerontol Int 2013;13(1):19-27.

61. Nieuwboer A. Cueing for freezing of gait in patients with Parkinson's disease: a rehabilitation perspective. Movement disorders : official journal of the Movement Disorder Society. 2008;23(S2):S475-S481.

62. Jette DU, Latham NK, Smout RJ, Gassaway J, Slavin MD, Horn SD. Physical therapy interventions for patients with stroke in inpatient rehabilitation facilities. Phys Ther. 2005;85(3):238-248.

63. Hochstenbach J, Mulder T, van Limbeek J, Donders R, Schoonderwaldt H. Cognitive decline following stroke: a comprehensive study of cognitive decline following stroke. J Clin Exp Neuropsychol. 1998;20(4):503-517.

64. Rasquin SM, Verhey FR, Lousberg R, Winkens I, Lodder J. Vascular cognitive disorders: memory, mental speed and cognitive flexibility after stroke. J Neurol Sci. 2002;203-204:115-119.

65. Masters RSW, Maxwell JP. Implicit motor learning, reinvestment and movement disruption: What you don't know won't hurt you? In. A.M. Williams \& N.J. Hodges (Eds.); 2004.

66. Maxwell JP, Masters RSW, Eves FF. The role of working memory in motor learning and performance. Conscious Cogn. 2003;12(3):376-402.

67. van Tilborg IA, Kessels RP, Hulstijn W. How should we teach everyday skills in dementia? A controlled study comparing implicit and explicit training methods. Clin Rehabil. 2011;25(7):638-648.

68. Jie LJ, Goodwin V, Kleynen M, Braun S, Nunns M, Wilson M. Analogy learning in Parkinson's disease: A proof-of-concept study. Int J Ther Rehabil. 2016;23(3):123-130. 
69. Subramanian SK, Massie CL, Malcolm MP, Levin MF. Does provision of extrinsic feedback result in improved motor learning in the upper limb poststroke? A systematic review of the evidence. Neurorehabil Neural Repair. 2010;24(2):113-124.

70. Cirstea CM, Ptito A, Levin MF. Feedback and cognition in arm motor skill reacquisition after stroke. Stroke. 2006;37(5):1237-1242.

71. Collen FM, Wade DT, Bradshaw CM. Mobility after stroke: reliability of measures of impairment and disability. Int Disabil Stud. 1990;12(1):6-9.

72. Gladstone DJ, Danells CJ, Black SE. The fugl-meyer assessment of motor recovery after stroke: a critical review of its measurement properties. Neurorehabil Neural Repair. 2002;16(3):232-240.

73. Masters RSW. Theoretical aspects of implicit learning in sport. Int J Sport Psychol. 2000.

74. Poolton JM, Masters RSW, Maxwell JP. The relationship between initial errorless learning conditions and subsequent performance. Hum Mov Sci. 2005;24(3):362-378.

75. Maxwell JP, Masters RSW, Kerr E, Weedon E. The implicit benefit of learning without errors. Q J Exp Psychol. 2001;54(4):1049-1068.

76. Blum L, Korner-Bitensky N. Usefulness of the Berg Balance Scale in stroke rehabilitation: a systematic review. Phys Ther. 2008;88(5):559-566.

77. Hsieh CL, Hsueh IP, Mao HF. Validity and responsiveness of the rivermead mobility index in stroke patients. Scand J Rehabil Med. 2000;32(3):140-142.

78. Nasreddine ZS, Phillips NA, Bédirian V, Charbonneau S, Whitehead V, Collin I, et al. The Montreal Cognitive Assessment, MoCA: a brief screening tool for mild cognitive impairment. J Am Geriatr Soc. 2005;53(4):695-699.

79. Wilson B, Cockburn J, Baddeley A. The Rivermead behavioural memory test (RBMT). Thames Valley Test Company, Bury St Edmunds. 1985.

80. Brickenkamp R, Zillmer E. The d2 Test of Attention. 1998. Goettingen, Hogrefe.

81. Zeni JA, Jr., Richards JG, Higginson JS. Two simple methods for determining gait events during treadmill and overground walking using kinematic data. Gait Posture. 2008;27(4):710-714.

82. Awad LN, Palmer JA, Pohlig RT, Binder-Macleod SA, Reisman DS. Walking speed and step length asymmetry modify the energy cost of walking after stroke. Neurorehabil Neural Repair. 2015;29(5):416-423.

83. Maxwell JP, Capio CM, Masters RSW. Interaction between motor ability and skill learning in children: Application of implicit and explicit approaches. European Journal of Sport Science. 2017;17(4):407-416.

84. Fulk GD, Ludwig M, Dunning K, Golden S, Boyne P, West T. Estimating Clinically Important Change in Gait Speed in People With Stroke Undergoing Outpatient Rehabilitation. Journal of neurologic physical therapy : JNPT. 2011;35(2).

85. Poolton JM, Masters RSW, Maxwell JP. The development of a culturally appropriate analogy for implicit motor learning in a Chinese population. Sport Psychol. 2007;21(4):375-375.

86. Kal E, Houdijk H, van der Kamp J, Verhoef M, Prosée R, Groet E, et al. Are the effects of internal focus instructions different from external focus 
instructions given during balance training in stroke patients? A double-blind randomized controlled trial. Clin Rehabil. 2018;33(2):207-221.

87. Bertram JE. Constrained optimization in human walking: cost minimization and gait plasticity. J Exp Biol. 2005;208(Pt 6):979-991.

88. Schmidt RA, Lee TD. Motor control and learning. a behavioral emphasis. Champaign, IL [etc.] Human Kinetics 1999 3rd ed; 1999.

89. Bijleveld-Uitman M, van de Port I, Kwakkel G. Is gait speed or walking distance a better predictor for community walking after stroke? J Rehabil Med. 2013;45(6):535-540.

90. Dobkin BH, Nadeau SE, Behrman AL, Wu SS, Rose DK, Bowden M, et al. Prediction of responders for outcome measures of locomotor Experience Applied Post Stroke trial. J Rehabil Res Dev. 2014;51(1):39-50.

91. Kal E, Winters M, van der Kamp J, Houdijk H, Groet E, van Bennekom C, et al. Is Implicit Motor Learning Preserved after Stroke? A Systematic Review with Meta-Analysis. PloS one. 2016;11(12):e0166376-e0166376.

92. Kleynen M, Moser A, Haarsma FA, Beurskens AJ, Braun SM. Physiotherapists use a great variety of motor learning options in neurological rehabilitation, from which they choose through an iterative process: a retrospective think-aloud study. Disability and rehabilitation. 2017:1-9.

93. Perry J, Garrett M, Gronley JK, Mulroy SJ. Classification of walking handicap in the stroke population. Stroke. 1995;26(6):982-989.

94. Dobkin BH. Clinical Practice. Rehabilitation after Stroke. N Engl J Med. 2005;352(16):1677-1684.

95. Krakauer JW. Motor learning: its relevance to stroke recovery and neurorehabilitation. Curr Opin Neurol. 2006;19(1):84-90.

96. Belda-Lois J-M, Mena-del Horno S, Bermejo-Bosch I, Moreno JC, Pons JL, Farina D, et al. Rehabilitation of gait after stroke: a review towards a top-down approach. J Neuroeng Rehabil. 2011;8(1):1-20.

97. Pennycott A, Wyss D, Vallery H, Klamroth-Marganska V, Riener R. Towards more effective robotic gait training for stroke rehabilitation: a review. $J$ Neuroeng Rehabil. 2012;9(1):65-65.

98. Moreira MC, de Amorim Lima AM, Ferraz KM, Benedetti Rodrigues MA. Use of virtual reality in gait recovery among post stroke patients - a systematic literature review. Disabil Rehabil Assist Technol. 2013;8(5):357-362.

99. Molina KI, Ricci NA, de Moraes SA, Perracini MR. Virtual reality using games for improving physical functioning in older adults: a systematic review. $J$ Neuroeng Rehabil. 2014;11(1):156-156.

100. Mehrholz J, Pohl M, Elsner B. Treadmill training and body weight support for walking after stroke. Cochrane Database Syst Rev. 2014(1):CD002840CD002840.

101. States RA, Salem Y, Pappas E. Overground gait training for individuals with chronic stroke: A Cochrane systematic review. Journal of neurologic physical therapy: JNPT. 2009.

102. Pollock A, Baer G, Campbell P, Choo PL, Forster A, Morris J, et al. Physical Rehabilitation Approaches for the Recovery of Function and Mobility After Stroke Major Update. Stroke. 2014;45(10):e202-e202.

103. Lee TD, Swinnen SP, Serrien DJ. Cognitive Effort and Motor Learning. Quest. 1994;46(3):328-344. 
104. Tse ACY, Wong TWL, Masters RSW. Examining motor learning in older adults using analogy instruction. Psychol Sport Exerc. 2017;28:78-84.

105. Rosner B. Hypothesis testing: two-sample inference-estimation of sample size and power for comparing two means. Fundamentals of biostatistics, 7th ed, Brooks/Cole, Cengage Learning. 2011:269-327.

106. Combs-Miller SA, Kalpathi Parameswaran A, Colburn D, Ertel T, Harmeyer A, Tucker L, et al. Body weight-supported treadmill training vs. overground walking training for persons with chronic stroke: a pilot randomized controlled trial. Clin Rehabil. 2014;28(9):873-884.

107. Tilson JK, Sullivan KJ, Cen SY, Rose DK, Koradia CH, Azen SP, et al. Meaningful gait speed improvement during the first 60 days poststroke: minimal clinically important difference. Phys Ther. 2010;90(2):196-208.

108. Berg K, Wood-Dauphine S, Williams JI, Gayton D. Measuring balance in the elderly: preliminary development of an instrument. Physiother Can. 1989;41(6):304-311.

109. Collen FM, Wade DT, Robb GF, Bradshaw CM. The Rivermead Mobility Index: a further development of the Rivermead Motor Assessment. Int Disabil Stud. 1991;13(2):50-54.

110. Fugl-Meyer AR, Jaasko L, Leyman I, Olsson S, Steglind S. The post-stroke hemiplegic patient. 1. a method for evaluation of physical performance. Scand $J$ Rehabil Med. 1975;7(1):13-31.

111. Kleynen M, Braun SM, Beurskens AJ, Verbunt JA, de Bie RA, Masters RS. Investigating the Dutch Movement-Specific Reinvestment Scale in people with stroke. Clin Rehabil. 2013;27(2):160-165.

112. Masters RSW, Polman RCJ, Hammond NV. Reinvestment: A dimension of personality implicated in skill breakdown under pressure. Pers Indiv Differ. 1993;14(5):655-666.

113. Shumway-Cook A, Taylor CS, Matsuda PN, Studer MT, Whetten BK. Expanding the scoring system for the Dynamic Gait Index. Phys Ther. 2013;93(11):1493-1506.

114. Schmid A, Duncan PW, Studenski S, Lai SM, Richards L, Perera S, et al. Improvements in Speed-Based Gait Classifications Are Meaningful. Stroke. 2007;38(7):2096-2100.

115. Jonsdottir J, Cattaneo D. Reliability and validity of the dynamic gait index in persons with chronic stroke. Arch Phys Med Rehabil. 2007;88(11):1410-1415.

116. Shumway-Cook A, Woollacott MH. Motor control: theory and practical applications. Lippincott Williams \& Wilkins; 1995.

117. Shumway-Cook A, Baldwin M, Polissar NL, Gruber W. Predicting the probability for falls in community-dwelling older adults. Phys Ther. 1997;77(8):812-819.

118. Matsuda PN, Taylor CS, Shumway-Cook A. Evidence for the validity of the modified Dynamic Gait Index across diagnostic groups. Phys Ther. 2014;94(7):996-996.

119. Wilson MR, Vine SJ, Bright E, Masters RSW, Defriend D, McGrath JS. Gaze training enhances laparoscopic technical skill acquisition and multi-tasking performance: a randomized, controlled study. Surg Endosc. 2011;25(12):37313739 . 
120. Orrell AJ, Masters RS, Eves FF. Reinvestment and movement disruption following stroke. Neurorehabil Neural Repair. 2009;23(2):177-183.

121. Denneman RPM, Kal EC, Houdijk H, Kamp Jvd. Over-focused? The relation between patients' inclination for conscious control and single- and dual-task motor performance after stroke. Gait \& posture. 2018;62:206-213.

122. Cooke A, Kavussanu M, McIntyre D, Boardley ID, Ring C. Effects of competitive pressure on expert performance: Underlying psychological, physiological, and kinematic mechanisms. Psychophysiology. 2011;48(8):11461156.

123. Vine SJ, Moore LJ, Cooke A, Ring C, Wilson MR. Quiet eye training: A means to implicit motor learning. Int J Sport Psychol. 2013;44(4):367-386.

124. Hilari K, Byng S, Lamping DL, Smith SC. Stroke and aphasia quality of life scale-39 (SAQOL-39). Stroke. 2003;34(8):1944-1950.

125. Hudak PL, Wright JG. The characteristics of patient satisfaction measures. Spine (Phila Pa 1976). 2000;25(24):3167-3177.

126. Saunders RP, Evans MH, Joshi P. Developing a Process-Evaluation Plan for Assessing Health Promotion Program Implementation: A How-To Guide. Health Promot Pract. 2005;6(2):134-147.

127. Berry D, Dienes ZP. Implicit learning: Theoretical and empirical issues. Psychology Press; 1993.

128. Verbeek JM, van Wegen E, van Peppen RPS, Hendriks HJM, Rietberg MB, van der Wees PJ, et al. KNGF Clinical Practice Guideline for Physical Therapy in patients with stroke. In: Royal Dutch Society for Physical Therapy (Koninklijk Nederlands Genootschap voor Fysiotherapie, KNGF); 2014.

129. Winstein CJ, Wolf SL, Dromerick AW, et al. Effect of a task-oriented rehabilitation program on upper extremity recovery following motor stroke: The icare randomized clinical trial. JAMA. 2016;315(6):571-581.

130. Kal E, van den Brink H, Houdijk H, van der Kamp J, Goossens PH, van Bennekom C, et al. How physical therapists instruct patients with stroke: an observational study on attentional focus during gait rehabilitation after stroke. Disability and rehabilitation. 2018;40(10):1154-1165.

131. Latham NK, Jette DU, Slavin M, Richards LG, Procino A, Smout RJ, et al. Physical Therapy During Stroke Rehabilitation for People With Different Walking Abilities. Arch Phys Med Rehabil. 2005;86(12):41-50.

132. Kleynen M, Jie LJ, Theunissen K, Rasquin SM, Masters RSW, Meijer K, et al. The immediate influence of implicit motor learning strategies on spatiotemporal gait parameters in stroke patients: a randomized within-subjects design. Clin Rehabil. 2019;33(4):619-630.

133. Kirtley C, Whittle MW, Jefferson RJ. Influence of walking speed on gait parameters. J Biomed Eng. 1985;7(4):282-288.

134. Fritz S, Lusardi M. White Paper: "Walking Speed: the Sixth Vital Sign". J Geriatr Phys Ther. 2009;32(2):2-5.

135. Jie LJ, Kleynen M, Beurskens A, Meijer K, Braun S. The Effects of Implicit and Explicit Motor Learning in Gait Rehabilitation of People After Stroke: Protocol for a Randomized Controlled Trial. JMIR Res Protoc. 2018;7(5):e142e142. 
136. Kelly VE, Janke AA, Shumway-Cook A. Effects of instructed focus and task difficulty on concurrent walking and cognitive task performance in healthy young adults. Exp Brain Res. 2010;207(1-2):65-73.

137. Pohl PS, McDowd JM, Filion DL, Richards LG, Stiers W. Implicit learning of a perceptual-motor skill after stroke. Phys Ther. 2001;81(11):1780-1789.

138. Tyson S, Connell L. The psychometric properties and clinical utility of measures of walking and mobility in neurological conditions: A systematic review. Clin Rehabil. 2009;23(11):1018-1033.

139. Dobkin BH. Strategies for stroke rehabilitation. Lancet Neurol. 2004;3(9):528536.

140. Perera S, Mody SH, Woodman RC, Studenski SA. Meaningful change and responsiveness in common physical performance measures in older adults. $J$ Am Geriatr Soc. 2006;54(5):743-749.

141. Bothwell LE, Greene JA, Podolsky SH, Jones DS. Assessing the Gold Standard - Lessons from the History of RCTs. New England Journal of Medicine. 2016.

142. Dobkin BH, Dorsch A. The promise of mHealth: Daily activity monitoring and outcome assessments by wearable sensors. Neurorehabil Neural Repair. 2011;25(9):788-798.

143. Senden R, Heyligers IC, Meijer K, Savelberg H, Grimm B. Acceleration-based motion analysis as a tool for rehabilitation: Exploration in simulated functional knee limited walking conditions. Am J Phys Med Rehabil. 2011;90(3):226-232.

144. Stevens A, Beurskens A, Köke A, Van Der Weijden T. The use of patientspecific measurement instruments in the process of goal-setting: A systematic review of available instruments and their feasibility. Clin Rehabil. 2013;27(11):1005-1019.

145. Stratford P GCWMBJ. Assessing disability and change on individual patients: a report of a patient specific measure. Physiother Can. 1995;47(4):258-263.

146. Jie LJ, Kleynen M, Beurskens A, Meijer K, Braun S. Implicit and explicit motor learning interventions have similar effects on walking speed in people after stroke: a randomized controlled single blind trial. Preprint at medRxiv doi: 01101/19008797. 2019.

147. Braun SM, van Haastregt JC, Beurskens AJ, Gielen AI, Wade DT, Schols JM. Feasibility of a mental practice intervention in stroke patients in nursing homes; a process evaluation. BMC Neurol. 2010.

148. Rothgangel A, Braun S, Smeets R, Beurskens A. Feasibility of a traditional and teletreatment approach to mirror therapy in patients with phantom limb pain: a process evaluation performed alongside a randomized controlled trial. Clin Rehabil. 2019.

149. Feigin VL, Norrving B, George MG, Foltz JL, Roth GA, Mensah GA. Prevention of stroke: a strategic global imperative. Nat Rev Neurol. 2016.

150. van Peppen RP, Kwakkel G, Wood-Dauphinee S, Hendriks HJ, Van der Wees PJ, Dekker J. The impact of physical therapy on functional outcomes after stroke: what's the evidence? Clin Rehabil. 2004;18(8):833-862.

151. Veerbeek JM, van Wegen E, van Peppen R, van der Wees PJ, Hendriks E, Rietberg M, et al. What Is the Evidence for Physical Therapy Poststroke? A Systematic Review and Meta-Analysis. PloS one. 2014;9(2):e87987-e87987.

152. Wiles R, Ashburn A, Payne S, Murphy C. Patients' expectations of recovery following stroke: a qualitative study. Disabil Rehabil 2002;24(16):841-850. 
153. Sirur R, Richardson J, Wishart L, Hanna S. The Role of Theory in Increasing Adherence to Prescribed Practice. Physiother Can. 2009;61(2):68-77.

154. Cumming TB, Marshall RS, Lazar RM. Stroke, cognitive deficits, and rehabilitation: still an incomplete picture. Int J Stroke. 2013;8(1):38-45.

155. Ferrari A, Ginis P, Nieuwboer A, Greenlaw R, Muddiman A, Chiari L. Handling Gait Impairments of Persons with Parkinson's Disease by Means of Real-Time Biofeedback in a Daily Life Environment. Paper presented at: International Conference on Smart Homes and Health Telematics2016.

156. Eames S, McKenna K, Worrall L, Read S. The Suitability of Written Education Materials for Stroke Survivors and Their Carers. Top Stroke Rehabil. 2003;10(3):70-83.

157. The QuickBoard [mobile application software]. Version 1.1.2. Memphis: The Quick Board [cited 2019 Jun 10].

158. MedApp - Grip op uw medicatie [mobile application software]. Version 0.34.1. Eindhoven (Netherlands): MedApp B.V. [cited 2019 Jun 10].

159. Oefen App Beroerte [mobile application software]. Version 1.5. Utrecht (Netherlands): De Hoogstraat Revalidatie [cited 2019 Jun 10].

160. Wright P, Mosser-Wooley D, Wooley B. Techniques \& tools for using color in computer interface design. Crossroads. 1997;3(3):3-6.

161. Boll F, Brune P. User Interfaces with a Touch of Grey? - Towards a Specific UI Design for People in the Transition Age. Procedia Comput Sci. 2015;63:511-516.

162. Morris JM. User interface design for older adults. Interact Comput. 1994;6(4):373-393.

163. Dodd C, Athauda R, Adam M. Designing user interfaces for the elderly: a systematic literature review. Paper presented at: Proceedings of the Australasian Conference on Information Systems2017.

164. British Design Council. The design process: what is the double diamond? https://www.designcouncil.org.uk/news-opinion/design-process-what-doublediamond. Published 2015. Accessed 20-12-2018.

165. Blomquist $\AA$, Arvola M. Personas in action: ethnography in an interaction design team. Paper presented at: Proceedings of the second Nordic conference on Human-computer interaction2002.

166. Ijsselsteijn W, Nap HH, de Kort Y, Poels K. Digital game design for elderly users. Paper presented at: Future Play; 2007; Toronto (Canada).

167. Meng Q, Lee MH. Design issues for assistive robotics for the elderly. Adv Eng Inform. 2006;20(2):171-186.

168. Burke JW, McNeill MDJ, Charles DK, Morrow PJ, Crosbie JH, McDonough SM. Optimising engagement for stroke rehabilitation using serious games. Vis Comput. 2009;25(12):1085-1085.

169. Flores E, Tobon G, Cavallaro E, Cavallaro FI, Perry JC, Keller T. Improving patient motivation in game development for motor deficit rehabilitation. Paper presented at: Proceedings of the 2008 International Conference on Advances in Computer Entertainment Technology2008.

170. Gerling KM, Schild J, Masuch M. Exergame design for elderly users: the case study of SilverBalance. Paper presented at: Proceedings of the 7th International Conference on Advances in Computer Entertainment Technology2010. 
171. Moffatt K, McGrenere J, Purves B, Klawe M. The participatory design of a sound and image enhanced daily planner for people with aphasia. Paper presented at: Proceedings of the SIGCHI conference on Human factors in computing systems2004.

172. Dehn DM, Van Mulken S. The impact of animated interface agents: a review of empirical research. Int J Hum Comput Stud. 2000;52(1):1-22.

173. Baig MM, GholamHosseini H, Connolly MJ. Mobile healthcare applications: system design review, critical issues and challenges. Australasian physical \& engineering sciences in medicine. 2015;38(1):23-38.

174. Peek ST, Luijkx KG, Rijnaard MD, Nieboer ME, van der Voort CS, Aarts S, et al. Older adults' reasons for using technology while aging in place. Gerontol. 2015;62(2):226-237.

175. Middleton A, Fritz SL, Lusardi M. Walking Speed: The Functional Vital Sign. J Aging Phys Act. 2015;23(2):314-322.

176. Lurie JD, Morgan TS. Pros and cons of pragmatic clinical trials. J Comp Eff Res. 2013;2(1):53-58.

177. Kampe te RRE. A qualitative study to identify factors, which influence the level of use of a real-time biofeedback system for home-based gait training in patients suffering from stroke (Unpublished MSc thesis) 2017.

178. Cox M, Loots F, Schillings J. De validiteit van het sensoren feedback-systeem "Stappy". Een hulpmiddel toegepast bij looptraining na een CVA 2018.

179. Verheijen F, Bewersdorff L. De hanteerbaarheid van het sensoren feedbacksysteem 'Stappy' ervaren door chronische CVA-patiënten (Unpublished BA thesis) 2019.

180. Jie LJ, van den Heuvel R, Braun S, Kleynen M. Looptraining met behulp van technologie: ervaringen van CVA-patiënten en therapeuten met het sensorenfeedback systeem 'Stappy'. Keypoint Een tijdschrift voor behandelaars in de neurorevalidatie. 2019.

181. Minary L, Trompette J, Kivits J, Cambon L, Tarquinio C, Alla F. Which design to evaluate complex interventions? Toward a methodological framework through a systematic review. BMC Medical Research Methodology. 2019;19(1):1-9.

182. Tarquinio C, Kivits J, Minary L, Coste J, Alla F. Evaluating complex interventions: Perspectives and issues for health behaviour change interventions. Psychology and Health. 2015;30(1):35-51.

183. Dal-Ré R, Janiaud P, Ioannidis JPA. Real-world evidence: How pragmatic are randomized controlled trials labeled as pragmatic? BMC Med. 2018;16(1):1-6.

184. Loudon K, Treweek S, Sullivan F, Donnan P, Thorpe KE, Zwarenstein M. The PRECIS-2 tool: Designing trials that are fit for purpose. BMJ (Online). 2015;350(May).

185. Kal E. Improving movement automaticity and dual-task performance in people after stroke. A change of focus? 2018.

186. van Abswoude F, Nuijen NB, van der Kamp J, Steenbergen B. Individual Differences Influencing Immediate Effects of Internal and External Focus Instructions on Children's Motor Performance. Research Quarterly for Exercise and Sport. 2018. 
187. Buszard T, Farrow D, Verswijveren SJJM, Reid M, Williams J, Polman R, et al. Working Memory Capacity Limits Motor Learning When Implementing Multiple Instructions. Front Psychol. 2017;8:1350-1350.

188. Arnstein SR. A Ladder Of Citizen Participation. J Am Plann Assoc. 1969.

189. Braun SM, Beurskens AJ, van Kroonenburgh SM, Demarteau J, Schols JM, Wade DT. Effects of mental practice embedded in daily therapy compared to therapy as usual in adult stroke patients in Dutch nursing homes: design of a randomised controlled trial. BMC Neurol. 2007;7(1):34-34.

190. Rothgangel AS, Braun S, Schulz RJ, Kraemer M, de Witte L, Beurskens A, et al. The PACT trial: PAtient Centered Telerehabilitation: Effectiveness of software-supported and traditional mirror therapy in patients with phantom limb pain following lower limb amputation: protocol of a multicentre randomised controlled trial. Journal of Physiotherapy. 2015;61(1):42-42.

191. Braun S, Kleynen M, Schols J, Schack T, Beurskens A, Wade D. Using mental practice in stroke rehabilitation: a framework. Clinical rehabilitation. 2008;22(7):579-591.

192. Kleynen M, Beurskens A, Olijve H, Kamphuis J, Braun S. Application of motor learning in neurorehabilitation: a framework for health-care professionals. Physiother Theory Pract 2018.

193. Rothgangel A, Braun S. Mirror Therapy: Practical Protocol for Stroke Rehabilitation. 2013.

194. Hunter SW, Divine A, Frengopoulos C, Odasso MM. A framework for secondary cognitive and motor tasks in dual-task gait testing in people with mild cognitive impairment. BMC Geriatr. 2018;18(1):1-7.

195. Zhu FF, Poolton JM, Wilson MR, Maxwell JP, Masters RSW. Neural coactivation as a yardstick of implicit motor learning and the propensity for conscious control of movement. Biological Psychology. 2011;87(1):66-73.

196. van Duijn T, Hoskens MCJ, Masters RSW. Analogy instructions promote efficiency of cognitive processes during hockey push-pass performance. Sport, Exercise, and Performance Psychology. 2019.

197. Poolton JM, Zachry TL. So you want to learn implicitly? Coaching and learning through implicit motor learning techniques. International Journal of Sports Science \& Coaching. 2007;2(1):67-78.

198. Smit R, Bartelse J, Bergkamp R, Dijkgraaf R, Harwig R, de Jong H, et al. Van voornemens naar voorsprong: kennis moet circuleren. Den Haag: Nederland Ondernemend Innovatieland Innovatieplatform;2009. https://www.rvo.nl/sites/default/files/bijlagen/Rapport\%20kennis\%20moet\%20 circuleren.pdf. Accessed 30-01-2020

199. Volksgezondheidenzorg.info. Prevalentie ziekte van Parkinson in huisartsenpraktijk. Rijksinstituut voor Volksgezondheid en Mileu. https://www.volksgezondheidenzorg.info/onderwerp/ziekte-vanparkinson/cijfers-context/huidige-situatie\#node-prevalentie-ziekte-vanparkinson-huisartsenpraktijk. Published 2020. Accessed 30-01-2020.

200. Volksgezondheidenzorg.info. Prevalentie en nieuwe gevallen beroerte in huisartsenpraktijk.

https://www.volksgezondheidenzorg.info/onderwerp/beroerte/cijferscontext/huidige-situatie\#node-prevalentie-en-nieuwe-gevallen-beroertehuisartsenpraktijk. Published 2020. Accessed 30-01-2020. 
201. Bravata DM, Smith-Spangler C, Sundaram V, Gienger AL, Lin N, Lewis R, et al. Using pedometers to increase physical activity and improve health: a systematic review. Jama. 2007;298(19):2296-2304. 